\title{
Sistemas estratificantes sobre álgebras hereditárias
}

\author{
Paula Andrea Cadavid Salazar
}

\author{
TESE APRESENTADA \\ $\mathrm{AO}$ \\ INSTITUTO DE MATEMÁTICA E ESTATÍSTICA \\ DA \\ UNIVERSIDADE DE SÃO PAULO \\ PARA \\ OBTENÇÃO DO TÍTULO \\ $\mathrm{DE}$ \\ DOUTOR EM CIÊNCIAS
}

\begin{abstract}
Programa: Matemática
Orientador: Prof. Dr. Eduardo do Nascimento Marcos

Durante o desenvolvimento deste trabalho o autor recebeu auxílio financeiro da CAPES e do CNPq
\end{abstract}

São Paulo, 25 de novembro de 2014 



\section{Sistemas estratificantes sobre álgebras hereditárias}

Esta versão da tese contém as correções e alterações sugeridas pela Comissão Julgadora durante a defesa da versão original do trabalho, realizada em 14/11/2012. Uma cópia da versão original está disponível no Instituto de Matemática e Estatística da

Universidade de São Paulo.

Comissão Julgadora:

- Prof. Dr. Eduardo do Nascimento Marcos (orientador) - IME-USP

- Profa. Dra. Maria Izabel Ramalho Martins - IME-USP

- Prof. Dr. Edson Ribeiro Alvares - UFPR

- Prof. Dr. Marcelo Americo Lanzilotta Mernies - Universidad de la República

- Prof. Dr. Viktor Vekkert - UFMG 

A Luisa y a Pablo 



\section{Agradecimentos}

Ao meu orientador Eduardo do Nascimento Marcos pela confiança e apoio durante esses anos. Sem ele este trabalho não teria sido possível.

À professora Maria Izabel Ramalho Martins por todo o tempo dedicado a me escutar, ler e corregir este trabalho.

Muito especialmente ao professor Marcelo Lanzilotta pela hospitalidade e atenção dispensada durante minhas visitas à Universidad de la República.

Ao professor Flavio Ulhoa Coelho pelas suas sugestões que ajudaram a melhorar este trabalho.

Ao meu colega Franciso Medeiros pelas discussões que esclareceram algumas das minhas dúvidas.

A minha família: Pablo e Luisa.

A meus queridos amigos Natalia, Sandro, Sandra, Cristian, Luana, Alejandra R., Javier e Alejanndra A.

Ao CNPq e a Capes, pelo apoio financeiro. 



\section{Resumo}

O principal tema deste trabalho é o estudo dos sistemas estratificantes sobre álgebras hereditárias. Um dos principais problemas é a construção de sistemas estratificantes completos cujos elementos sejam todos módulos regulares, sendo este problema resolvido para álgebras hereditárias do tipo mansa e as álgebras de Kronecker generalizadas.

Para as álgebras hereditárias de tipo mansa exibimos um limitante para o tamanho dos sistemas estratificantes formados só de módulos regulares e, usando tal limitante, concluímos que não é possível que tais sistemas estratificantes sejam completos. Para as álgebras de Kronecker e as álgebras de Kronecker generalizadas concluimos que nenhum sistema estratificante sobre esta álgebra pode ter elementos regulares e construímos todos os possíveis sistemas estratificantes completos sobre esta álgebra.

Definimos o conceito de sequência especial de um módulo inclinante, estabelecemos que todo módulo inclinante tem uma sequência especial e estudamos quando uma sequência, de dois e três somandos diretos de um módulo inclinante, é uma sequência especial.

Palavras-chave: Sistema estratificante, álgebra hereditária, módulo inclinante, sequência excepcional. 



\section{Abstract}

The main topic of this work is the study of stratifying systems over hereditary algebras. One of the main questions to be considered is the construction of complete stratifying systems whose elements are regular modules. We solve this problem for tame hereditary algebras and for the Kronecker generalized algebras.

In the case of tame hereditary algebras, we obtain a bound for the size of the stratifying systems composed only by regular modules and, by using this bound, we conclude that such stratifying systems can not be complete. For the Kronecker and for Kronecker the generalized algebras we conclude that no stratifing system over this algebra can have regular elements. Next we construct all possible complete stratifying systems over this algebra.

Furthermore, we define the notion of special sequence of a tilting module and we establish that all tilting modules have an special ordenation. Also we study when an sequence of two and three direct summands of an tilting module, is a special ordenation.

Keywords: stratifying systems, hereditary algebra, tilting module, exceptional sequence. 



\section{Sumário}

1 Preliminares 1

1.1 Carcases e álgebras de caminho . . . . . . . . . . . . . . . . 2

1.2 Representações e módulos . . . . . . . . . . . . . . . . . . . . . 4

1.3 Sequências de Auslander-Reiten . . . . . . . . . . . . . . . . . . . . 7

1.4 Os funtores de translação $\tau$ e $\tau^{-1} \ldots \ldots \ldots \ldots \ldots \ldots \ldots$

1.5 Sobre o grupo de Grothendieck . . . . . . . . . . . . . . . . . . . . . 11

1.6 Sobre álgebras hereditárias . . . . . . . . . . . . . . . . . . . 13

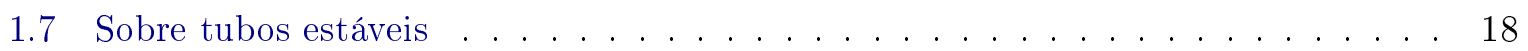

2 Sistemas estratificantes sobre álgebras hereditárias $\quad 23$

2.1 Sistemas estratificantes . . . . . . . . . . . . . . . . . . . 24

2.2 Módulos estândares . . . . . . . . . . . . . . . . . . . . . . . . . . . . 27

2.3 Sequências excepcionais . . . . . . . . . . . . . . . . . 31

3 Sistemas estratificantes de módulos regulares sobre álgebras hereditárias euclidianas

3.1 Sistemas estratificantes de módulos regulares sobre álgebras hereditárias euclidianas 43

3.2 Sistemas estratificantes sobre álgebras de tipo $\Delta\left(\widetilde{\mathbb{A}}_{p, q}\right) \ldots \ldots \ldots \ldots \ldots$

4 Sistemas estratificantes via módulos inclinantes em álgebras hereditárias $\quad 75$

$\begin{array}{lr}\text { Referências Bibliográficas } & 89\end{array}$

$\begin{array}{lr}\text { Referências Bibliográficas } & 89\end{array}$

$\begin{array}{ll}\text { Índice Remissivo } & 91\end{array}$ 



\section{Introdução}

Os sistemas estratificantes foram introduzidos, em [7], por K. Erdmann e C. Sáenz, como uma generalização dos módulos estândares. Em [7], os autores mostram que para um sistema estratificante $\theta$ a categoria $\mathcal{F}(\theta)$, dos módulos filtrados por $\theta$, é equivalente à categoria dos módulos filtrados pelos módulos estândares sobre uma álgebra estandarmente estratificada apropriada. Além disso, mostram que a categoria $\mathcal{F}(\theta)$ gera um módulo tal que seu anel de endomorfismos é uma álgebra estandarmente estratificada.

Em [10, 11], E. Marcos, O. Mendoza e C. Sáenz, introduzem os sistemas estratificantes via módulos relativamente projetivos e os sistemas estratificantes via módulos relativamente simples, respectivamente, e mostram que as três noções de sistema estratificante são equivalentes. Em [12], os mesmos autores, encontraram um limitante para a dimensão finitística de uma álgebra estandarmente estratificada usando a teoria dos sistemas estratificantes.

Por outro lado, em [13], E. Marcos, O. Mendoza, C. Sáenz e R. Zuazua, introduzem a forma quadrática $q_{\theta}$, que depende de um sistema estratificante $\theta$, usando-a para estabelecer uma condição, suficiente e necessária, para que a categoria $\mathcal{F}(\theta)$ seja finita.

Nosso objetivo é estudar sistemas estratificantes sobre álgebras hereditárias. Neste trabalho, mostramos que existe uma relação estreita entre os sistemas estratificantes e os módulos inclinantes. Usando esta relação obtemos um limitante para o tamanho destes, e deste modo introduzimos a noção de sistemas estratificantes completos.

Uma questão de interesse é a existência de um sistema estratificante completo formado somente de módulos regulares. Se $A=K Q$, onde $Q$ é um carcás acíclico e conexo, então se $\bar{Q}$ for um diagrama de Dynkin ou um diagrama euclidiano, a resposta é não. Mesma resposta vale se $A$ for uma álgebra de Kronecker ou uma álgebra de Kronecker generalizada.

Por outro lado, o conceito de sequência exepcional foi introduzido por Gordentsev e Rudakov, em [9], com o intuito de estudar fibrados vetoriais sobre $\mathbb{P}^{2}$. Tais sequências tem sido usadas por vários autores no estudo das categorias derivadas de variedades algebraicas (ver por exemplo $[4,16])$. Crawely-Boevey considerou a noção correspondente no contexto das representações de um carcás e, em [6], demonstrou que se $K$ é um corpo algebricamente fechado e $A$ é uma $K$ álgebra hereditária, existe uma ação transitiva do grupo de tranças gerado por $s-1$ elementos sobre o conjunto de sequências excepcionais completas de tamanho $s$. Depois Ringel, em [14], mostrou que o mesmo resultado vale se $A$ for uma álgebra de Artin qualquer.

Usando o fato de que, sobre uma álgebra hereditária, as noções de sistema estratificante e sequência excepcional são equivalentes e os resultados de [6], calculamos explicitamente todos os sistemas estratificantes completos sobre uma álgebra de Kronecker (ou Kronecker generalizada) e construímos uma família de sistemas estratificantes completos com um número maximal de 
elementos regulares sobre a álgebra canônica $\triangle\left(\widetilde{\mathbb{A}}_{p, q}\right)$.

A tese está organizada da seguinte forma: o Capítulo 1, destina-se à revisão de conceitos, definições, teoremas e notações usadas na teoria de representações de álgebras. Também tratamos das álgebras hereditárias e algumas das suas propriedades. Finalmente, definimos alguns tipos de componentes no carcás de Auslander-Reiten que serão usadas no Capítulo 3.

No Capítulo 2, definimos os sistemas estratificantes e descrevemos algumas das suas propriedades. Definimos também o conjunto dos módulos estândares, as álgebras estandarmente estratificadas e uma classe particular delas: as álgebras quase-hereditárias. Além disso, usando o grupo de Grothendieck para um sistema estratificante, fazemos uma demonstração simples de que uma álgebra estandarmente estratificada é quase-hereditária se, e somente se, tem dimensão global finita. Estabelecemos uma relação entre os sistemas estratificantes sobre álgebras hereditárias e os módulos inclinantes e, usando tal relação, encontramos um limitante para o tamanho destes. Introduzimos o conceito de sequência especial para um módulo. Também, usamos resultados em [6], para calcular especificamente todos os sistemas estratificantes completos sobre a álgebra de Kronecker e sobre as álgebras de Kronecker generalizadas.

No Capítulo 3, demonstramos que não é possível construir um sistema estratificante completo cujos elementos sejam módulos regulares e encontramos um limitante para o tamanho de um sistema estratificante com tais características. Mais ainda, mostramos que não é possível construir tal sistema estratificante em um tipo mais geral de álgebras: as álgebras disfarçadas de tipo euclideano. Construímos uma família de sistemas estratificantes completos com um número maximal de elementos regulares sobre a álgebra canônica $\triangle\left(\widetilde{\mathbb{A}}_{p, q}\right)$.

Finalmente, no Capítulo 4, mostramos que dado um módulo inclinante parcial básico sobre uma álgebra hereditária existe uma sequência especial de seus somandos diretos. Depois, encontramos condições suficientes e necessárias para que uma sequência de dois módulos seja uma sequência especial. Por último, tratamos o caso de uma sequência de três módulos. 


\section{Capítulo 1}

\section{Preliminares}

Vale enfatizar que ao longo deste texto a palavra álgebra significa álgebra associativa, com 1 e de dimensão finita sobre um corpo algebricamente fechado. Alguns dos resultados mencionados neste trabalho valem num contexto mais geral, mas por simplicidade vamos nos restringir a este.

Faremos aqui uma breve descrição das noções de álgebras associativas de dimensão finita que utilizaremos ao longo desta tese. Para consultar detalhes citamos aos Capítulos I, II, III, VII, VIII e o Apêndice A de [2] assim como o Cap. XIII em [17].

Se $A$ é uma $K$-álgebra de dimensão finita, então o módulo $A_{A}$ admite uma decomposição da forma

$$
A=P_{1} \oplus P_{2} \oplus \ldots \oplus P_{n}
$$

onde cada $P_{i}=e_{i} A$ é um $A$-módulo projetivo indecomponível e $e_{1}, e_{2}, \ldots, e_{n}$ são idempotentes primitivos, dois a dois ortogonais, e tais que $1=e_{1}+e_{2}+\cdots+e_{n}$. O conjunto $\left\{e_{1}, e_{2}, \cdots, e_{n}\right\}$ é chamado de um conjunto completo de idempotentes primitivos ortogonais.

Dizemos que $A$ é uma álgebra básica se $e_{i} A \supsetneqq e_{j} A$, quando $i \neq j$, para todo $i, j=1, \ldots, n$. Além disso dizemos que $A$ é conexa (ou indecomponível) se não pode ser decomposta como soma direta de duas álgebras, ou equivalentemente, se 0 e 1 são seus únicos idempotentes centrais.

Denotamos por Mod $A$ a categoria cujos objetos são os $A$-módulos à direita e cujos morfismos são os homomorfismos de $A$-módulos e por $\bmod A$ a subcategoria plena de $\operatorname{Mod} A$ cujos objetos são os $A$-módulos finitamente gerados.

O principal objetivo na teoria de representações de álgebras é o estudo das categorias de módulos. Mas, sem perda de generalidade, podemos estudar as categorias de módulos sobre álgebras básicas. Isto devido a que, como consequência dos Teoremas de Morita, se uma $K$ álgebra $A$ é de dimensão finita então existe uma $K$-álgebra $B$ básica tal que $\bmod A$ e $\bmod B$ são equivalentes. 


\subsection{Carcases e álgebras de caminho}

Seja $A$ uma álgebra de dimensão finita e básica sobre um corpo algebricamente fechado. Nesta seção daremos uma caracterização de $A$ em termos de estruturas chamadas carcases. Para isto, começaremos definindo carcases, veremos como se podem construir álgebras a partir deles e para finalizar enunciaremos o Teorema de Gabriel, que proporciona a caracterização mencionada acima. A prova de tal teorema pode ser vista no Capítulo II de [2].

Um carcás é uma quádrupla $Q=\left(Q_{0}, Q_{1}, c, f\right)$ formada de dois conjuntos $Q_{0}$ (cujos elementos chamamos vértices) e $Q_{1}$ (cujos elementos chamamos flechas), e duas funções $c: Q_{1} \rightarrow Q_{0}$ e $f: Q_{1} \rightarrow Q_{0}$.

Dada uma flecha $\alpha \in Q_{1}$, se $c(\alpha)=a$ e $f(\alpha)=b$ dizemos que o começo de $\alpha$ é $a$ e que o final de $\alpha$ é $b$. Denotamos esta situação por $\alpha: a \rightarrow b$. O carcás $Q=\left(Q_{0}, Q_{1}, c, f\right)$ pode ser denotado por $Q=\left(Q_{0}, Q_{1}\right)$ ou simplesmente por $Q$.

Dizemos que $Q=\left(Q_{0}, Q_{1}\right)$ é finito se os conjuntos $Q_{0}$ e $Q_{1}$ são finitos. Todos os carcases que consideraremos aqui serão finitos, exceto o carcás de Auslander-Reiten que definiremos na seção 1.4.

O grafo subjacente $\bar{Q}$ do carcás $Q$ é um grafo obtido de $Q$ sem considerar a orientação das flechas, isto é, $\bar{Q}$ é um grafo com os mesmos vértices de $Q$ e tal que existe uma aresta entre os vértices $a$ e $b$ em $\bar{Q}$ se existe uma flecha $\alpha: a \rightarrow b$ ou uma flecha $\beta: b \rightarrow a$. Dizemos que o carcás $Q$ é conexo se o grafo subjacente $\bar{Q}$ é conexo. Sejam $Q=\left(Q_{0}, Q_{1}, c, f\right)$ e $a, b \in Q_{0}$. Um caminho de comprimento $\ell \geq 1$ com começo em $a$ e final em $b$ (ou simplesmente de $a$ para $b$ ) é uma sequência

$$
\left(a\left|\alpha_{1}, \alpha_{2}, \ldots, \alpha_{\ell}\right| b\right)
$$

onde $\alpha_{k} \in Q_{1}$, para $1 \leq k \leq \ell, c\left(\alpha_{1}\right)=a, f\left(\alpha_{k}\right)=c\left(\alpha_{k+1}\right)$, para cada $1 \leq k \leq \ell-1$, e $f\left(\alpha_{\ell}\right)=b$. Denotamos tal caminho por $\alpha_{1} \alpha_{2} \ldots \alpha_{\ell}$. Além disso, a cada vértice $a \in Q_{0}$ associamos um caminho de comprimento $\ell=0$ que chamamos caminho trivial e que denotamos por $\epsilon_{a}$ ou por $(a \| a)$.

Um caminho de comprimento $\ell \geq 1$ é chamado ciclo quando seu começo e seu final coincidem. Um carcás que não contém ciclos é chamado de acíclico.

Dados um carcás $Q$ e um corpo $K$ uma álgebra de caminhos $K Q$ é uma $K$-álgebra cujo $K$-espaço vetorial subjacente tem como base o conjunto de todos os caminhos de comprimento $\ell \geq 0$ em $Q$.

A seguir definimos o produto em $K Q$ nos elementos da sua base. Se $\gamma_{1}=\left(a\left|\alpha_{1}, \alpha_{2}, \ldots, \alpha_{\ell}\right| b\right)$ e $\gamma_{2}=\left(c\left|\beta_{1}, \beta_{2}, \ldots, \beta_{k}\right| d\right)$, então

$$
\gamma_{1} \gamma_{2}=\left\{\begin{array}{l}
\left(a\left|\alpha_{1}, \alpha_{2}, \ldots, \alpha_{\ell}, \beta_{1}, \beta_{2}, \ldots, \beta_{k}\right| d\right), \text { se } b=c \\
0, \text { caso contrário. }
\end{array}\right.
$$


Tal produto é estendido por linearidade para qualquer elemento de $K Q$.

Uma relação em $Q$ é uma combinação $K$-linear de caminhos, de comprimento maior do que um, que tem o mesmo início e o mesmo final. Isto é, uma relação $\rho$ é um elemento de $K Q$ da forma

$$
\rho=\sum_{i=1}^{m} \lambda_{i} \omega_{i}
$$

onde $\lambda_{i} \in K$ (não todos nulos), $\ell\left(\omega_{i}\right)>1$ para $i=1, \ldots, m$, e existem $a, b \in Q_{0}$ tais que $c\left(\omega_{i}\right)=a$ e $f\left(\omega_{i}\right)=b$ para $i=1, \ldots, m$.

Sejam $Q$ um carcás finito e $R$ o ideal de $K Q$ gerado pelas flechas de $Q$. Dizemos que um ideal bilateral $I$ de $K Q$ é admissível se existe um inteiro $m \geq 2$, tal que

$$
R^{m} \subseteq I \subseteq R^{2}
$$

É conhecido que se $Q$ é finito e $I$ é um ideal admissível de $K Q$, então existe um conjunto finito de relações $\left\{\rho_{1}, \rho_{2}, \ldots, \rho_{s}\right\}$ tais que $I=\left\langle\rho_{1}, \rho_{2}, \ldots, \rho_{s}\right\rangle$. O par $(Q, I)$ é chamado carcás com relações ou carcás limitado e a álgebra quociente $K Q / I$ associada ao par $(Q, I)$ é chamada de álgebra de caminhos do carcás com relações $(Q, I)$.

Até aqui dado um carcás $Q$ definimos a álgebra $K Q$ a partir de $Q$. Agora, assumindo que $A$ é uma $K$-álgebra de dimensão finita, básica e $K$ um corpo algebricamente fechado vamos construir o carcás $Q_{A}$ a partir de $A$ e veremos de que forma $A$ e $K Q_{A}$ estão relacionadas.

Sejam $K$ um corpo algebricamente fechado, $A$ uma $K$-álgebra básica com $\operatorname{dim}_{K} A<\infty$, e $\left\{e_{1}, e_{2}, \ldots, e_{n}\right\}$ um conjunto completo de idempotentes ortogonais primitivos. O carcás ordinário de $A$, que denotaremos por $Q_{A}$, é definido da seguinte forma:

1. Os vértices de $Q_{A}$ são $v_{1}, v_{2}, \ldots, v_{n}$ que estão em correspondência bijetora com os idempotentes $e_{1}, e_{2}, \ldots, e_{n}$.

2. Dados dois vértices $v_{i}$ e $v_{j}$ em $\left(Q_{A}\right)_{0}$, fixamos uma base para o $K$-espaço vetorial

$$
e_{i}\left(\operatorname{rad} A / \operatorname{rad}^{2} A\right) e_{j}
$$

As flechas $\alpha: v_{i} \rightarrow v_{j}$ estão em correspondência bijetora com os vetores de tal base.

A seguir enunciamos o Teorema de Gabriel que estabelece uma relação entre álgebras e seus carcases.

Teorema de Gabriel I 1.1.1. Seja A uma K-álgebra básica e conexa de dimensão finita, onde $K$ é um corpo algebricamente fechado. Então existe um ideal admissivel I de $K Q_{A}$ tal que $A \cong K Q_{A} / I$. Além disso, se $\psi: K Q \rightarrow A$ é um epimorfismo de $K$-álgebras tal que Nuc $\psi$ é um ideal admissivel de $K Q_{A}$ então $Q=Q_{A}$.

Um epimorfismo como no teorema acima se chama uma apresentação de $A$. 


\subsection{Representações e módulos}

Na seção anterior, vimos como algumas álgebras podem ser descritas em termos de carcases com relações. Agora vamos visualizar as representações de uma álgebra, isto é, os seus módulos, através do correspondente carcás com relações. Todos os conceitos aqui apresentados podem ser encontrados no Capítulo III de [2].

Ao longo desta seção assumimos que $A$ é uma $K$-álgebra de dimensão finita, básica e $K$ um corpo algebricamente fechado e consideraremos em todos os casos carcases finitos.

Seja $Q$ um carcás finito. Definimos uma representação $K$-linear ou, simplesmente, uma representação $M$ do carcás $Q$ da seguinte forma:

1. Para cada vértice $a \in Q_{0}$ associamos um $K$-espaço vetorial $M_{a}$.

2. Para cada flecha $\alpha: a \rightarrow b$ em $Q_{1}$ associamos uma aplicação $K$-linear $\varphi_{\alpha}: M_{a} \rightarrow M_{b}$.

Denotamos tal representação como $M=\left(M_{a}, \varphi_{\alpha}\right)_{a \in Q_{0}, \alpha \in Q_{1}}$, ou simplesmente como $M=$ $\left(M_{a}, \varphi_{\alpha}\right)$; e dizemos que é de dimensão finita se cada espaço vetorial $M_{a}$ é de dimensão finita.

Sejam $M=\left(M_{a}, \varphi_{\alpha}\right)$ e $M^{\prime}=\left(M_{a}^{\prime}, \varphi_{\alpha}^{\prime}\right)$ duas representações $K$-lineares de $Q$. Um morfismo (de representações) $f: M \rightarrow M^{\prime}$ é uma família $f=\left(f_{a}\right)_{a \in Q_{0}}$ de $K$-aplicações lineares $f_{a}$ : $M_{a} \rightarrow M_{a}^{\prime}$, as quais são compatíveis com as aplicações $\varphi_{\alpha}$. Isto é, para cada flecha $\alpha: a \rightarrow b$, vale que

$$
\varphi_{\alpha}^{\prime} f_{a}=f_{b} \varphi_{\alpha}
$$

ou, equivalentemente, cada um dos seguintes quadrados comutam:

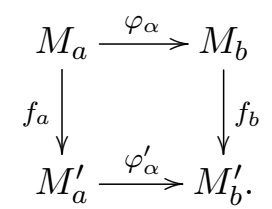

Sejam $f: M \rightarrow M^{\prime}$ e $g: M^{\prime} \rightarrow M^{\prime \prime}$ dois morfismos de representações de $Q$, onde $f=\left(f_{a}\right)_{a \in Q_{0}}$ e $g=\left(g_{a}\right)_{a \in Q_{0}}$. Definimos a composição $g f: M \longrightarrow M^{\prime \prime}$ como sendo a família $g f=\left(g_{a} f_{a}\right)_{a \in Q_{0}}$.

Desse modo, temos definido a categoria $\operatorname{Rep}(Q)$ de representações $K$-lineares de $Q$. Denotamos por $\operatorname{rep}(Q)$ a subcategoria plena de $\operatorname{Rep}(Q)$, que consiste das representações de dimensão finita.

Seja $M=\left(M_{a}, \varphi_{\alpha}\right)$ uma representação de $Q$. Para um caminho não trivial

$$
\omega=\left(a\left|\alpha_{1}, \alpha_{2}, \ldots, \alpha_{\ell}\right| b\right)
$$


em $Q$, a avaliação de $M$ em $\omega$ é a função $K$-linear $\varphi_{\omega}: M_{a} \rightarrow M_{b}$ definida por

$$
\varphi_{\omega}=\varphi_{\alpha_{1}} \varphi_{\alpha_{2}} \ldots \varphi_{\alpha_{\ell}}
$$

Estendemos esta definição para combinações lineares de caminhos. Isto é, se $\rho=\sum_{i=1}^{m} \lambda_{i} \omega_{i}$ é uma relação, então a avaliação de $M$ em $\rho$ é $\varphi_{\rho}=\sum_{i=1}^{m} \lambda_{i} \varphi_{\omega_{i}}$.

Exemplo 1.2.1. Seja $A=K Q$, onde $Q$ é o carcás

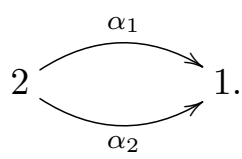

$A$ é conhecida como a álgebra de Kronecker. Uma representação $M$ de $Q$ é dada por

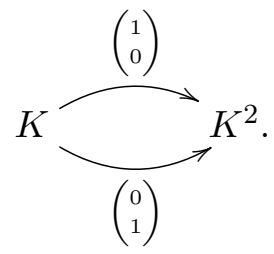

Outra representação $M^{\prime}$ esta dada por

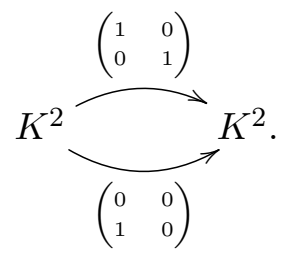

Temos um morfismo $M \longrightarrow M^{\prime}$ definido por

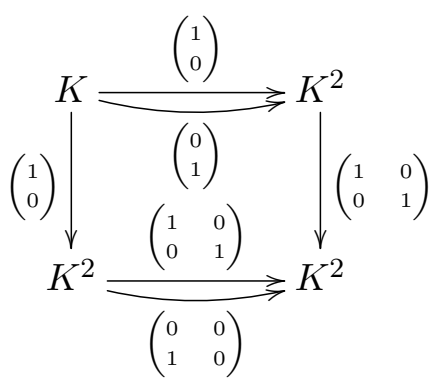

Pois temos que

$$
\left(\begin{array}{ll}
1 & 0 \\
0 & 1
\end{array}\right)\left(\begin{array}{l}
1 \\
0
\end{array}\right)=\left(\begin{array}{ll}
1 & 0 \\
0 & 1
\end{array}\right)\left(\begin{array}{l}
1 \\
0
\end{array}\right) \text { e }\left(\begin{array}{ll}
1 & 0 \\
0 & 1
\end{array}\right)\left(\begin{array}{l}
0 \\
1
\end{array}\right)=\left(\begin{array}{ll}
0 & 0 \\
1 & 0
\end{array}\right)\left(\begin{array}{l}
1 \\
0
\end{array}\right)
$$

Seja $I$ um ideal admissível de $K Q$. Dizemos que a representação $M=\left(M_{a}, \varphi_{\alpha}\right)$ de $Q$ satisfaz 
as relações em $I$ quando $\varphi_{\rho}=0$, para todo elemento $\rho \in I$. Notemos que se $I$ é gerado pelo conjunto de relações $\left\{\rho_{1}, \rho_{2}, \ldots, \rho_{s}\right\}$, a representação $M$ satisfaz as relações de $I$ se, e somente se, $\varphi_{\rho_{i}}=0$, para todo $1 \leq i \leq s$.

Uma representação de $(Q, I)$ é uma representação de $Q$ que satisfaz as relações de $I$. Denotamos por $\operatorname{Rep}_{K}(Q, I)$ a subcategoria de $\operatorname{Rep}_{K}(Q)$ cujos objetos são as representações de $(Q, I)$ e por $\operatorname{rep}_{K}(Q, I)$ a subcategoria de $\operatorname{rep}_{K}(Q)$ cujos objetos são as representações de dimensão finita de $(Q, I)$.

Se $A$ é uma $K$-álgebra básica de dimensão finita sobre o corpo $K$ algebricamente fechado então, pelo Teorema de Gabriel, $A$ é isomorfa a uma álgebra de caminhos dada por um carcás com relações $(Q, I)$, isto é, $A \cong K Q / I$. A seguinte proposição diz que o estudo da categoria $\operatorname{Mod} A$ é equivalente ao da categoria $\operatorname{Rep}(Q, I)$.

Teorema 1.2.2. Seja $A=K Q / I$, onde $Q$ é um carcás finito e conexo e I é um ideal admissível de $K Q$. Então existe uma equivalência $F$ de categorias

$$
F: \operatorname{Mod} A \stackrel{\simeq}{\longrightarrow} \operatorname{Rep}_{K}(Q, I)
$$

cuja restrição é uma equivalência entre as categorias

$$
F: \bmod A \stackrel{\simeq}{\longrightarrow} \operatorname{rep}_{K}(Q, I) .
$$

Demonstração. Descreveremos o funtor $F: \operatorname{Mod} A \longrightarrow \operatorname{Rep}_{K}(Q, I)$. Para isto diremos como age nos objetos e nos morfismos de $\operatorname{Mod} A$.

Sejam $a \in Q_{0}$ e $\alpha \in Q_{1}$. Denotaremos por $e_{a}$ e por $\bar{\alpha}$ as classes de $\epsilon_{a}$ e de $\alpha$ em $K Q / I$, respectivamente.

Se $M$ é um $A$-módulo, definimos $F(M)=\left(M_{a}, \varphi_{\alpha}\right)$, onde $M_{a}=M e_{a}$ e para $\alpha: a \longrightarrow b$, seja $\varphi_{\alpha}: M_{a} \longrightarrow M_{b}$ dada por $\varphi_{\alpha}(x)=x \bar{\alpha}$, para todo $x \in M_{a}$.

Se $f: M \rightarrow M^{\prime}$ é um homomorfismo de $A$-módulos, então definimos $F(f)=\left(f_{a}\right)_{a \in Q_{0}}$, onde $f_{a}$ é a restrição de $f$ a $M_{a}=M e_{a}$. É fácil verificar que $F$ é uma equivalência de categorias.

Notemos que na demonstração do teorema anterior é construido o funtor que faz com que as duas categorias sejam equivalentes. Tal teorema permite a identificação os $A$-módulos com as representações $K$-lineares de $(Q, I)$ e vice-versa. Diante disto, abusaremos da linguagem não distinguindo, muitas vezes, os $K Q / I$ - módulos das representações de $(Q, I)$.

$\mathrm{O}$ último teorema tem muitas consequências interessantes; em particular permite conhecer de forma explícita os módulos simples, os módulos projetivos indecomponíveis e os módulos injetivos indecomponíveis. 


\subsection{Sequências de Auslander-Reiten}

Nesta seção vamos enunciar um resultado que surgiu na década dos 70 e que influenciou definitivamente o desenvolvimento da teoria de representações de álgebras. Trabalhando basicamente com álgebras de Artin (uma generalização das álgebras de dimensão finita) M. Auslander e I. Reiten introduziram a noção de sequências quase-cindidas (também chamadas sequências de Auslander-Reiten).

Posteriormente os morfismos que aparecem nas mencionadas sequências foram usados por C. M. Ringel para definir um carcás, conhecido como o carcás de Auslander-Reiten, que proporciona muita informação sobre a categoria $\bmod A$.

Detalhes dos conceitos e resultados aqui mencionados podem ser achados no Capítulo IV de [2].

Começaremos definindo os conceitos relacionados tais como morfismo irredutível, sequência quase-cindida, entre outros.

Sejam $K$ um corpo algebricamente fechado, $A$ uma $K$-álgebra de dimensão finita sobre $K$ e $M, N, L A$-módulos em $\bmod A$. Então:

1. Seja $h: M \rightarrow N$ um homomorfismo de $A$-módulos. Dizemos que $h$ é uma seção (ou um monomorfismo que cinde) se existe um homomorfismo de $A$-módulos $s: N \longrightarrow M$ tal que $s h=1_{M}$. De outro lado, dizemos que $h$ é uma retração (ou um epimorfismo que cinde) se existe um homomorfismo de $A$-módulos $r: N \longrightarrow M$ tal que $h r=1_{N}$.

2. Um homomorfismo $h: M \longrightarrow N$ de $A$-módulos é irredutível se $h$ não é seção, nem retração e se $h=h_{1} h_{2}$ implica que $h_{1}$ é retração ou que $h_{2}$ é seção.

Notemos que um morfismo irredutível em mod $A$ é um monomorfismo próprio ou um epimorfismo próprio. Isto é, um morfismo irredutível é um monomorfismo não isomorfismo ou um epimorfismo não isomorfismo. De fato, suponhamos que $f: X \longrightarrow Y$ é irredutível, não é um epimorfismo próprio e que $f=j p$ é a sua fatoração canônica. Então $j$ não é um epimorfismo que cinde e portanto $p$ é um monomorfismo que cinde. Logo $f$ é um monomorfismo próprio.

Dizemos que uma sequência exata curta

$$
0 \longrightarrow L \stackrel{f}{\longrightarrow} M \stackrel{g}{\longrightarrow} N \longrightarrow 0, \text { em } \bmod A
$$

é uma sequência quase-cindida ou uma sequência de Auslander-Reiten se $L$ e $N$ são $A$-módulos indecomponíveis e $f$ e $g$ são morfismos irredutíveis.

Terorema de Auslander-Reiten 1.3.1. $\quad$ 1. Seja M um A-módulo indecomponível não projetivo. Então existe uma sequência quase-cindida, única a menos de equivalências de sequências exatas, da forma

$$
0 \longrightarrow M^{\prime} \longrightarrow E \longrightarrow M \longrightarrow 0, \quad \text { em } \bmod A
$$


2. Seja L um A-módulo indecomponível não injetivo. Então existe uma sequência quasecindida, única a menos de equivalências de sequências exatas, da forma

$$
0 \longrightarrow L \longrightarrow F \longrightarrow L^{\prime} \longrightarrow 0, \quad \text { em } \bmod A
$$

Sejam $X$ e $Y A$-módulos indecomponíveis. O $K$-espaço vetorial

$$
\operatorname{Irr}(X, Y)=\operatorname{rad}_{A}(X, Y) / \operatorname{rad}_{A}^{2}(X, Y)
$$

onde $\operatorname{rad}_{A}(X, Y)$ é o $K$-espaço vetorial dos homomorfismos não invertíveis de $X$ em $Y$ e $\operatorname{rad}_{A}^{2}(X, Y)$ é o $K$-espaço vetorial dos homomorfismos da forma $g f \operatorname{com} f \in \operatorname{rad}_{A}(X, Z)$ e $g \in \operatorname{rad}_{A}(Z, Y)$, para algum $A$-módulo $Z$, é denominado espaço dos morfismos irredutíveis de $X$ em $Y$. Como $X$ e $Y$ são $A$-módulos indecomponíveis, então a dimensão de $\operatorname{Irr}(X, Y)$ é igual ao número máximo de homomorfismos irredutíveis de $X$ em $Y$, que são linearmente independentes. O carcás de Auslander-Reiten da categoria $\bmod A$, denotado por $\Gamma(\bmod A)$, é definido da seguinte forma:

1. Os vértices de $\Gamma(\bmod A)$ são as classes de isomorfismos $[M]$ de $A$-módulos indecomponíveis $M$.

2. Se $[M]$ e $[N]$ são dois vértices de $\Gamma(\bmod A)$, correspondentes aos $A$-módulos indecomponíveis $M$ e $N$, o número de flechas de $[M]$ para $[N]$ é igual à dimensão do espaço vetorial $\operatorname{Irr}(M, N)$.

Exemplo 1.3.2. Seja $A=K Q$, onde $Q$ é o carcás

$$
Q: \quad 1<2 \longrightarrow 3 \text {. }
$$

A seguinte é uma lista completa dos $A$-módulos indecomponíveis:

$$
\begin{aligned}
& S_{1}=(K \longleftarrow 0 \longrightarrow 0) \quad=P_{1} \\
& S_{2}=(0 \longleftarrow K \longrightarrow 0) \quad=I_{2} \\
& S_{3}=(0 \longleftarrow 0 \longrightarrow K) \quad=P_{3} \\
& P_{2}=\left(K \stackrel{I d_{K}}{\longleftarrow} K \stackrel{I d_{K}}{\longleftrightarrow} K\right) \\
& I_{1}=\left(K \stackrel{I d_{K}}{\longleftarrow} K \longrightarrow 0\right) \\
& I_{3}=\left(0 \longleftarrow K \stackrel{I d_{K}}{\longrightarrow} K\right) \text {. }
\end{aligned}
$$

Onde $S_{i}, P_{i}$ e $I_{i}$ para $i=1,2,3$, denotam os $A$-módulos simples indecomponíveis, os $A$ módulos projetivos indecomponíveis e os $A$-módulos injetivos indecomponíveis, respectivamente.

As sequências de Auslander-Reiten sobre $A$ são as sequências abaixo 


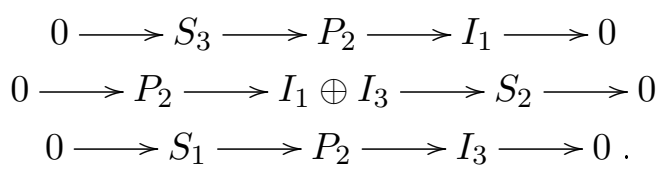

E, finalmente, o carcás de Auslander-Reiten de $A$ é o carcás

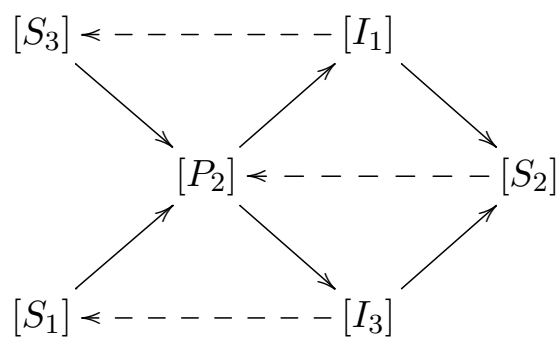

\subsection{Os funtores de translação $\tau$ e $\tau^{-1}$}

Nesta seção vamos definir a translação de Auslander-Reiten. Os detalhes da construção e resultados aqui mencionados podem ser encontrados no Capítulo IV em [2].

Sejam $K$ um corpo e $A$ uma $K$-álgebra de dimensão finita sobre $K$. Consideremos as categorias $\bmod A$ e $\bmod A^{o p}$ e indicamos resumidamente a construção das suas categorias projetivamente e injetivamente estáveis. Para cada par $(M, N)$ de objetos em $\bmod A$ denotamos por $\mathcal{P}(M, N)$ (respectivamente por $\mathcal{I}(M, N)$ ) o subconjunto de $\operatorname{Hom}_{A}(M, N)$ formado pelos morfismos que se fatoram através de algum $A$-módulo projetivo (respectivamente, injetivo). É conhecido que a classe $\mathcal{P}=\{\mathcal{P}(M, N) / M, N \in \bmod A\}$ é um ideal da categoria $\bmod A$. Analogamente para a classe $\mathcal{I}=\{\mathcal{I}(M, N) / M, N \in \bmod A\}$.

Com o ideal $\mathcal{P}$ é construída a categoria quociente $\underline{\bmod } A=\bmod A / \mathcal{P}$, denominada categoria projetivamente estável.

Para recordar, os objetos em $\underline{\bmod } A$ são os objetos de $\bmod A$ e dados dois $A$-módulos $M$ e $N$ os morfismos de $M$ para $N$ em $\underline{\bmod } A$ são os elementos do quociente de $K$-espaços vetoriais $\underline{\operatorname{Hom}}_{A}(M, N)=\operatorname{Hom}_{A}(M, N) / \mathcal{P}(M, N)$.

Analogamente, através do ideal $\mathcal{I}$, é definida a categoria quociente $\overline{\bmod } A=\bmod A / \mathcal{I}$, denominada categoria injetivamente estável. É conhecido que o funtor $D=\operatorname{Hom}_{K}(\ldots, K)$ é uma dualidade entre as categorias $\bmod A$ e $\bmod A^{o p}$. Porém, $D$ induz uma dualidade, também denotada por $D$, de $\underline{\bmod } A \longrightarrow \overline{\bmod } A^{o p}$.

O objetivo agora é definir uma outra dualidade entre as categorias $\underline{\bmod } A$ e $\overline{\bmod } A^{o p}$. Para tal, consideremos $M$ em $\bmod A$ e seja

$$
P_{1} \stackrel{d_{1}}{\longrightarrow} P_{0} \stackrel{d_{0}}{\longrightarrow} M \longrightarrow 0
$$


uma apresentação projetiva minimal de $M$. Isto é, uma sequência exata tal que $d_{0}: P_{0} \longrightarrow M$ e $d_{1}: P_{1} \longrightarrow$ Nuc $d_{0}$ são coberturas projetivas de $M$ e de Nuc $d_{0}$, respectivamente.

O funtor $\operatorname{Hom}_{A}(\ldots, A)$, permite construir o $A^{o p}$-módulo à direita $\operatorname{Hom}_{A}\left(\operatorname{Conuc} d_{1}, A\right)$, que é definido como o transposto de $M$ que é denotado por $\operatorname{Tr} M$ (que é único a menos de isomorfismo). Com essa construção pode-se definir um funtor dualidade, denotado por $\mathrm{Tr}$, $\operatorname{Tr}: \underline{\bmod } A \longrightarrow \overline{\bmod } A^{o p}$, que a cada $M$ em $\underline{\bmod } A$ associa $\operatorname{Tr} M \in \overline{\bmod } A^{o p}$.

Com os funtores $D: \underline{\bmod } A^{o p} \longrightarrow \overline{\bmod }\left(A^{o p}\right)^{o p}=\overline{\bmod } A$ e $\operatorname{Tr}: \underline{\bmod } A \longrightarrow \overline{\bmod } A^{o p}$, são definidos, por composição, os funtores de translação de Auslander-Reiten

$$
\tau: \underline{\bmod } A \longrightarrow \overline{\bmod } A \text { e seu inverso } \tau^{-1}: \overline{\bmod } A \longrightarrow \underline{\bmod } A
$$

da seguinte forma

$$
\tau=\operatorname{DTr} \quad \text { e } \quad \tau^{-1}=\operatorname{Tr} D
$$

Por último observamos que o Teorema 1.3.1 garante a existência de uma sequência exata quasecindida $0 \longrightarrow M^{\prime} \longrightarrow E \longrightarrow M \longrightarrow 0$, sendo $M$ um $A$-módulo indecomponível não projetivo. $\mathrm{O}$ módulo $M^{\prime}$ é construído de forma que $M^{\prime}=\tau M=D \operatorname{Tr} M$. No caso da existência da sequência quase-cindida $0 \longrightarrow L \longrightarrow F \longrightarrow L^{\prime} \longrightarrow 0$, com $L$ indecomponível não injetivo, o módulo $L^{\prime}=$ $\tau^{-1} L=\operatorname{Tr} D L$.

Para finalizar esta seção vamos enunciar um teorema muito conhecido, e que usaremos ao longo deste trabalho: as Fórmulas de Auslander.

Teorema 1.4.1. Sejam $K$ um corpo algebricamente fechado, $A$ uma $K$-álgebra de dimensão finita e $M$ e $N$ objetos em $\bmod A$. Então, temos os seguintes isomorfismos

$$
\operatorname{Ext}_{A}^{1}(M, N) \cong D \underline{\operatorname{Hom}}_{A}\left(\tau^{-1} N, M\right) \cong D \overline{\operatorname{Hom}}_{A}(N, \tau M),
$$

que são funtoriais nas duas variáveis.

Corolário 1.4.2. Sejam $K$ um corpo algebricamente fechado, $A$ uma $K$-álgebra de dimensão finita e $M$ e $N$ objetos em $\bmod A$. Então:

1. Se $\operatorname{pd} M \leq 1$, então existe um isomorfismo K-linear

$$
\operatorname{Ext}_{A}^{1}(M, N) \cong D \operatorname{Hom}_{A}\left(\tau^{-1} N, M\right) .
$$

2. Se $\operatorname{pd} N \leq 1$, então existe um isomorfismo K-linear

$$
\operatorname{Ext}_{A}^{1}(M, N) \cong D \operatorname{Hom}_{A}(N, \tau M) .
$$




\subsection{Sobre o grupo de Grothendieck}

Sejam $K$ um corpo algebricamente fechado, $A$ uma $K$-álgebra de dimensão finita sobre $K$ e $\left\{e_{1}, e_{2}, \ldots, e_{n}\right\}$ um conjunto completo de idempotentes primitivos ortogonais de $A$. Se $A=$ $\oplus_{i=1}^{n} e_{i} A$, então os $A$-módulos $P_{i}=e_{i} A$ e $I_{i}=D\left(A e_{i}\right), i=1, \ldots, n$, são respectivamente os $A$ módulos projetivos indecomponíveis e os $A$-módulos injetivos indecomponíveis que, além disso são, respectivamente, a cobertura projetiva e a envolvente injetiva do simples $S_{i} \cong$ top $P_{i} \cong$ $S o c I_{i}$.

Lembramos que, dado $M \in \bmod A$ e um elemento idempotente $e \in A$, vale que

$$
\operatorname{Hom}_{A}(e A, M) \cong M e .
$$

Em particular, $\operatorname{End}_{A}\left(S_{i}\right) \cong e_{i}(\operatorname{top} A) e_{i}$.

Seja $\boldsymbol{e}=\left(e_{1}, e_{2}, \ldots, e_{n}\right)$ um conjunto completo e ordenado de idempotentes primitivos ortogonais de $A$. A ordem fixada $e$ determina uma ordenação no conjunto dos $A$-módulos simples $S_{i}$, bem como dos $A$-módulos projetivos indecomponíveis $P_{i}$ e dos $A$-módulos injetivos indecomponíveis $I_{i}$. Com tal ordem podemos definir o vector dimensão de um $A$-módulo como segue.

Definição 1.5.1. Sejam $\boldsymbol{e}=\left(e_{1}, e_{2}, \ldots, e_{n}\right)$ um conjunto completo e ordenado de idempotentes primitivos ortogonais de $A$ e $M \in \bmod A$. Definimos o vetor dimensão de $M$ como o vetor de $\mathbb{Z}^{n}$, denotado por $\underline{\operatorname{dim}} M$, dado por

$$
\underline{\operatorname{dim}} M=\left[\begin{array}{c}
\operatorname{dim}_{K} M e_{1} \\
\vdots \\
\operatorname{dim}_{K} M e_{n}
\end{array}\right] .
$$

Assim o vetor $\underline{\operatorname{dim}} S_{i}$, de cada simples $S_{i}$, é o $i$-ésimo vetor da base canônica de $\mathbb{Z}^{n}$. De outro lado, como temos que $\operatorname{Hom}_{A}\left(P_{i}, M\right) \cong M e_{i}$ e que

$$
D \operatorname{Hom}_{A}\left(M, I_{i}\right) \cong D \operatorname{Hom}_{A^{o p}}\left(A e_{i}, M\right) \cong D\left(e_{i} D M\right) \cong D(D M) e_{i} \cong M e_{i},
$$

podemos reescrever o vetor $\underline{\operatorname{dim}} M$ das seguintes formas:

$$
\underline{\operatorname{dim}} M=\left[\begin{array}{c}
\operatorname{dim}_{K} \operatorname{Hom}_{A}\left(P_{1}, M\right) \\
\vdots \\
\operatorname{dim}_{K} \operatorname{Hom}_{A}\left(P_{n}, M\right)
\end{array}\right]=\left[\begin{array}{c}
\operatorname{dim}_{K} \operatorname{Hom}_{A}\left(M, I_{1}\right) \\
\vdots \\
\operatorname{dim}_{K} \operatorname{Hom}_{A}\left(M, I_{n}\right)
\end{array}\right] .
$$

Observação 1.5.2. Se $0 \longrightarrow L \longrightarrow M \longrightarrow N \longrightarrow 0$ é uma sequência exata em $\bmod A$, então $\underline{\operatorname{dim}} M=\underline{\operatorname{dim}} L+\underline{\operatorname{dim}} N$. 
Definição 1.5.3. Sejam $K$ um corpo algebricamente fechado e A uma K-álgebra, básica e de dimensão finita sobre $K$. Chama-se grupo de Grothendieck de $\bmod A$ o grupo abeliano $K_{0}(A)=\mathcal{F} / \mathcal{F}^{\prime}$, onde $\mathcal{F}$ é o grupo abeliano livre cuja base é o conjunto de classes de isomorfismos $\tilde{M}$ dos módulos $M$ em $\bmod A$ e $\mathcal{F}^{\prime}$ é o subgrupo de $\mathcal{F}$ gerado pelos elementos $\tilde{M}-\tilde{L}-\tilde{N}$ correspondentes às sequências exatas $0 \longrightarrow L \longrightarrow M \longrightarrow N \longrightarrow 0$ em $\bmod A$.

Denotaremos por $[M]$ a imagem da classe de isomorfismo $\tilde{M}$ do módulo $M$ pelo epimorfismo canônico de grupos $\mathcal{F} \longrightarrow \mathcal{F} / \mathcal{F}^{\prime}$ e por $\left[M: S_{i}\right]$ o número de fatores de composição de $M$ isomorfos a $S_{i}$.

A proposição abaixo fornece uma caracterização do grupo $K_{0}(A)$, cuja demonstração pode ser encontrada em [17].

Proposição 1.5.4. Sejam A uma $K$-álgebra, básica, de dimensão finita sobre $K, \boldsymbol{e}=\left(e_{1}, \ldots, e_{n}\right)$ um conjunto completo e ordenado de idempotentes primitivos ortogonais de $A$ e $\left\{S_{1}, \ldots, S_{n}\right\}$ um conjunto completo de classes de isomorfismo de A-módulos simples à direita. Então, o grupo de Grothendieck $K_{0}(A)$ de $\bmod A$ é um grupo abeliano livre com uma base dada por $\left\{\left[S_{1}\right], \ldots,\left[S_{n}\right]\right\}$ e existe um único isomorfismo de grupos dado por

$$
\underline{\operatorname{dim}}: K_{0}(A) \longrightarrow \mathbb{Z}^{n}
$$

tal que, $\underline{\operatorname{dim}}([M])=\underline{\operatorname{dim}} M$ para todo A-módulo $M$.

Usando esta base para $K_{0}(A)$ obtemos o seguinte corolário que usaremos com frequência.

Corolário 1.5.5. Seja $M$ um A-módulo. Então, para cada $j=1, \ldots, n$, vale que

$$
\left[M: S_{j}\right]=\operatorname{dim}_{K} \operatorname{Hom}_{A}\left(P_{j}, M\right)=\operatorname{dim}_{K} \operatorname{Hom}_{A}\left(M, I_{j}\right) .
$$

Em particular, usando os vetores dimensão dos $A$-módulos projetivos (ou injetivos) indecomponíveis obtemos uma matriz de coeficientes inteiros, que é conhecida como matriz de Cartan de $A$.

Definição 1.5.6. Sejam A uma $K$-álgebra, básica de dimensão finita sobre $K$ e $\boldsymbol{e}=\left(e_{1}, \ldots, e_{n}\right)$ um conjunto completo e ordenado de idempotentes primitivos ortogonais de $A$. A matriz de Cartan de $A$ é a matriz $n \times n$

$$
C_{A}=\left[\begin{array}{ccc}
c_{11} & \cdots & c_{1 n} \\
\vdots & \ddots & \vdots \\
c_{1 n} & \cdots & c_{n n}
\end{array}\right]
$$


onde $c_{i j}=\operatorname{dim}_{K} e_{j} A e_{i}$ para $i, j=1, \ldots, n$.

Observemos que, como $e_{j} A e_{i} \cong \operatorname{Hom}_{A}\left(P_{i}, P_{j}\right) \cong \operatorname{Hom}_{A}\left(I_{i}, I_{j}\right)$, cada entrada $c_{j i}$ de $C_{A}$ corresponde ao número de homomorfismos linearmente independentes de $P_{i}$ a $P_{j}$ e ao número de homomorfismos linearmente independentes de $I_{i}$ a $I_{j}$.

\subsection{Sobre álgebras hereditárias}

Nesta seção vamos mencionar várias caracterizações das álgebras hereditárias e algumas das suas propriedades. Mais detalhes dos assuntos aqui abordados podem ser encontrados nos Capítulos VII e VIII de [2].

Proposição 1.6.1. Sejam $K$ um corpo algebricamente fechado e $A$ uma $K$-álgebra de dimensão finita sobre K. Então são equivalentes as seguintes condições:

1. A é uma álgebra hereditária.

2. Todo A-módulo tem dimensão projetiva menor ou igual a 1.

3. Para cada par de A-módulos $M$ e $N$,

$$
\operatorname{Ext}_{A}^{i}(M, N)=0, \text { para todo } i \geq 2 \text {. }
$$

4. Todo submódulo de um A-módulo projetivo é projetivo.

5. Todo quociente de um A-módulo injetivo é injetivo.

6. Para cada par de A-módulos $M$ e $N$, $\operatorname{Ext}_{A}^{2}(M, N)=0$.

Vamos observar agora que uma álgebra hereditária também pode ser caracterizada em termos do seu carcás ordinário. É o que conta o teorema abaixo.

Teorema 1.6.2. (1.7 Cap.VII em [2]) Sejam $K$ um corpo algebricamente fechado e $A$ uma $K$-álgebra de dimensão finita sobre $K$. Então $A$ é hereditária se, e somente se, $A \cong K Q$, onde $Q$ é um carcás finito e acíclico.

Vamos adotar para o carcás de Auslander-Reiten a seguinte convenção: os vértices correspondentes a os $A$-módulos projetivos indecomponíveis serão chamados vértices projetivos e a os vértices correspondentes a os $A$-módulos injetivos indecomponíveis vértices injetivos.

Usamos esta convenção para descrever como as álgebras hereditárias podem ser caracterizadas em termos de seu carcás de Auslander-Reiten na proposição abaixo. 
Proposição 1.6.3. (1.10 Cap. VII em [2]) Sejam K um corpo algebricamente fechado e A uma $K$-álgebra de dimensão finita sobre K. Então as seguintes condições são equivalentes:

1. A é hereditária.

2. Em $\Gamma(\bmod A)$ os predecessores dos vértices projetivos são vértices projetivos.

3. Em $\Gamma(\bmod A)$ os sucessores dos vértices injetivos são vértices injetivos.

Um $A$ módulo indecomponível $M$ (com $A$ uma álgebra não necessariamente hereditária) é dito pós-projetivo se é da forma $\tau^{-k} P_{i}$, onde $P_{i}$ é um projetivo indecomponível e $k \geq 0$. Por outro lado, $M$ é dito pré-injetivo se é da forma $\tau^{l} I_{j}$, onde $I_{j}$ é um injetivo indecomponível e $l \geq 0$. O módulo $M$ é dito regular se não for pós-projetivo ou pré-injetivo. É importante ressaltar que, se $M$ é um módulo pós-projetivo ou pré-injetivo, então $\operatorname{End}_{A}(M) \cong K$ e $\operatorname{Ext}_{A}^{1}(M, M)=0$.

Se $A$ é uma álgebra hereditária de tipo de representação infinito então o seu carcás de Auslander-Reinten é a união disjunta

$$
\Gamma(\bmod A)=\mathcal{P}(A) \cup \mathcal{R}(A) \cup \mathcal{I}(A),
$$

onde $\mathcal{P}(A)$ é uma componente conexa formada pelos módulos pós-projetivos (conhecida como a componente pós-projetiva), $\mathcal{R}(A)$ é uma família de componentes conexas formadas por módulos regulares (conhecidas como componentes regulares) e $\mathcal{I}(A)$ é uma componente conexa formada por módulos pré-injetivos (conhecida como componente pré-injetiva). Além disso, valem as seguintes igualdades:

$$
\operatorname{Hom}_{A}(\mathcal{R}(A), \mathcal{P}(A))=0, \operatorname{Hom}_{A}(\mathcal{Q}(A), \mathcal{P}(A))=0, \operatorname{Hom}_{A}(\mathcal{Q}(A), \mathcal{R}(A))=0
$$

Se $A$ for uma álgebra hereditária conexa de tipo de representação finito $\Gamma(\bmod A)$ contém só uma componente conexa e não existem $A$-módulos regulares.

Uma classificação completa de todas as álgebras que são de tipo de representação finito (isto é, as que têm um número finito de classes de isomorfismo de módulos indecomponíveis) é conhecida e é tratada no importante Teorema de Gabriel II que está estreitamente relacionado com os diagramas de Dynkin que listaremos a seguir.

\section{Diagramas de Dynkin}

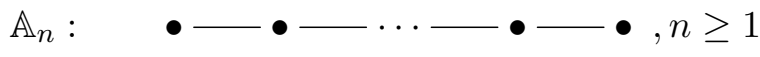



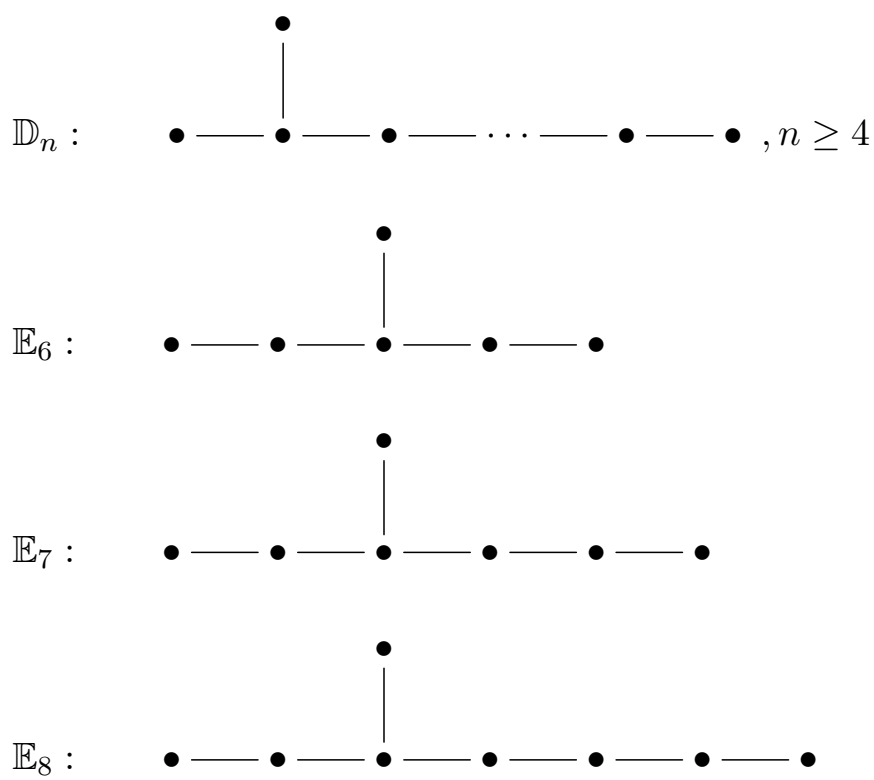

Conhecendo a lista de tais diagramas enunciamos o Teorema de Gabriel.

Teorema de Gabriel II 1.6.4. (5.10 Cap. VII em [2]) Sejam K um corpo algebricamente fechado e $A \cong K Q$ uma $K$-álgebra básica e conexa. Então $A$ é de tipo de representação finita se, e somente se, $\bar{Q}$ é um diagrama de Dynkin.

$\mathrm{Na}$ teoria de representações de álgebras joga um papel muito importante a classe das álgebras hereditárias que são de tipo de representação infinito e minimais com respeito a esta propriedade. Estas são justamente as álgebras de tipo euclidiano, isto é as álgebras de caminho $K Q$, onde $Q$ é um carcás conexo e acíclico cujo grafo subjacente $\bar{Q}$ é um dos grafos na seguinte lista:

\section{Diagramas euclidianos}
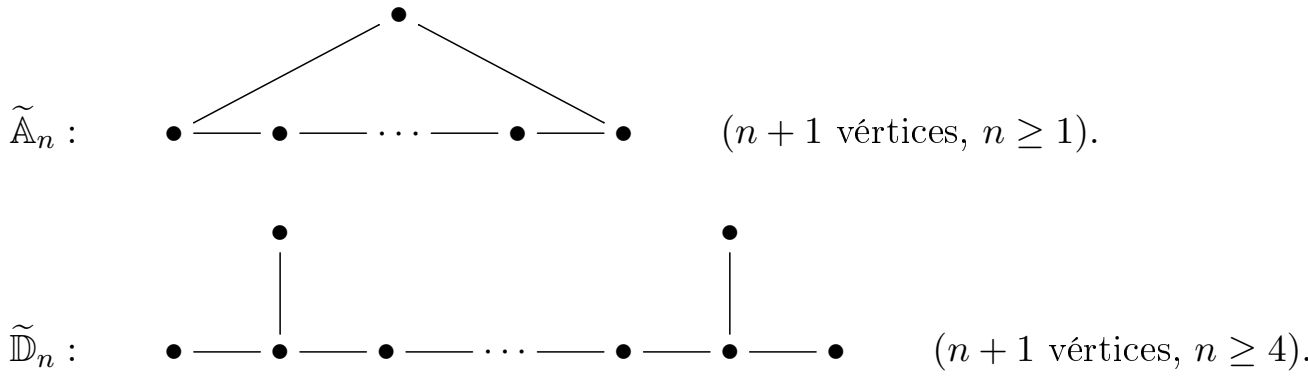


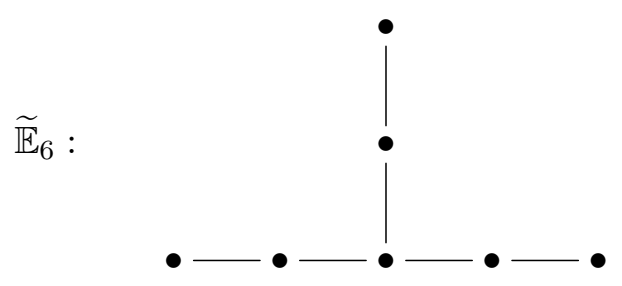

$\widetilde{\mathbb{E}}_{7}: \quad \bullet-\bullet-\bullet-\bullet-\bullet-\bullet-\bullet-\bullet$

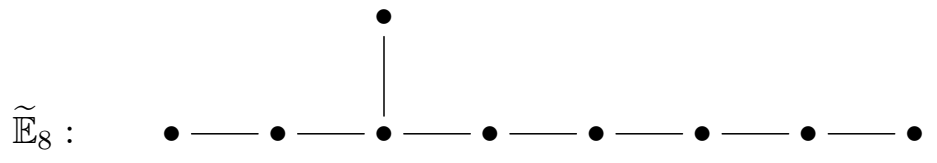

A seguinte lista de carcases é particularmente importante no estudo das álgebras euclidianas.

\section{Diagramas euclidianos canonicamente orientados}
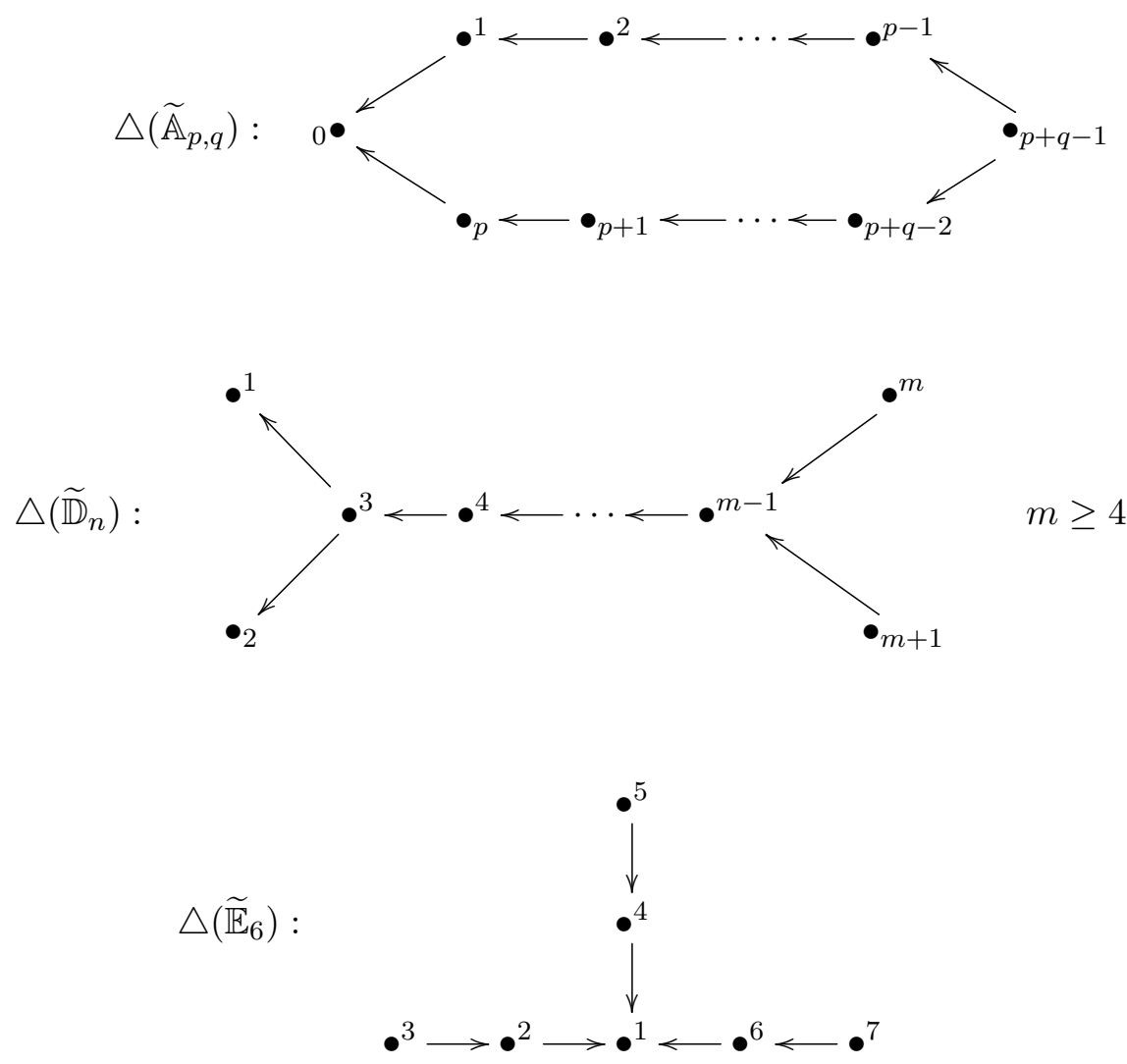


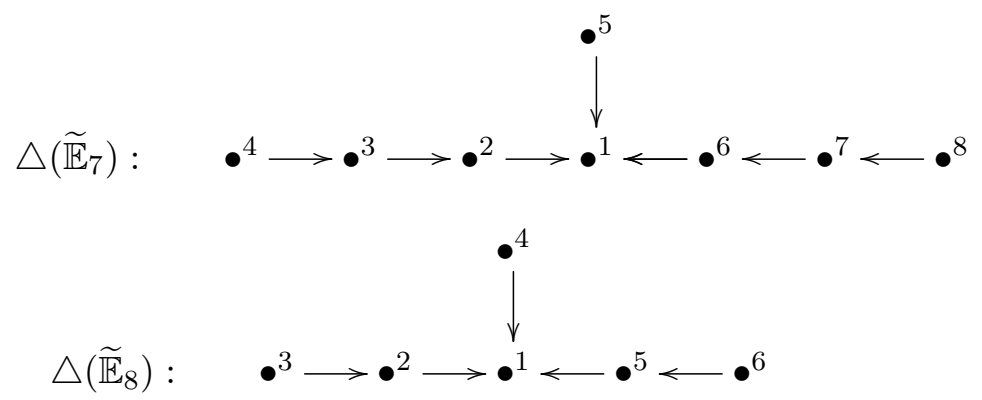

A importância de tais carcases esta dada pelo seguinte teorema.

Teorema 1.6.5. (2.1 (a) Cap. XIII em [2]) Sejam Q um carcás cujo grafo subjacente é euclidiano e $A=K Q$ a álgebra de caminhos de $Q$. Então existe um carcás euclidiano canonicamente orientado $\Delta$ tal que $Q$ é obtido de $\Delta$ por sequência finita de reflexões e A é uma álgebra inclinada de tipo $\Delta$.

Definição 1.6.6. Sejam A uma $K$-álgebra de dimensão global finita e $C_{A}$ a matriz de Cartan de $A$ com respeito ao conjunto completo de idempotentes ortogonais e primitivos $\left\{e_{1}, \ldots, e_{n}\right\}$ de A. A a matriz de Coxeter de A é a matriz

$$
\Phi_{A}=-C_{A} C_{A}^{-1} \in M_{n}(\mathbb{Z}) .
$$

O homomorfismo de grupos $\Phi_{A}: \mathbb{Z}^{n} \longrightarrow \mathbb{Z}^{n}$, definido pela fórmula $\Phi_{A}(\boldsymbol{x})=\Phi_{A} \cdot \boldsymbol{x}$, para todo $\boldsymbol{x}=\left(x_{1}, \ldots, x_{n}\right)^{t} \in \mathbb{Z}^{n}$, é chamada a transformação de Coxeter de $A$.

Proposição 1.6.7. Sejam $Q$ um carcás acíclico, $A=K Q$ sua álgebra de caminhos e $\Phi_{A}$ : $\mathbb{Z}^{n} \longrightarrow \mathbb{Z}^{n}$ a transformação de Coxeter de A. Então:

$\underline{\operatorname{dim}} \tau^{m} M=\Phi_{A}^{m}(\underline{\operatorname{dim}} M)$, para $m \geq 0$. 


\subsection{Sobre tubos estáveis}

No Capítulo 3 estudaremos acerca de sistemas estratificantes em componentes do carcás de Auslander-Reiten conhecidas como tubos estáveis, por isso, nesta seção, definiremos tais componentes e enunciamos propriedades que iremos usar.

Mais detalhes sobre os tópicos desta seção podem ser encontrados no Cap. VIII de [2] e no Cap. X de [17].

Sejam $Q=\left(Q_{0}, Q_{1}\right)$ um carcás e $x \in Q_{0}$. Denotaremos por $x^{+}$o conjunto dos sucessores imediatos de $x$, ou seja, $x^{+}=\left\{y \in Q_{0} / \exists \alpha \in Q_{1}, \alpha: x \longrightarrow y\right\}$. Por outro lado, denotaremos por $x^{-}$o conjunto dos predecessores imediatos de $x$, ou seja, $x^{-}=\left\{y \in Q_{0} / \exists \alpha \in Q_{1}, \alpha: y \longrightarrow x\right\}$. Diremos que $Q$ é localmente finito quando para todo $x \in Q_{0}$ os conjuntos $x^{+}$e $x^{-}$são finitos.

Definição 1.7.1. Sejam $\Gamma$ um carcás localmente finito e sem ciclos orientados e $\tau: \Gamma_{0}^{\prime} \longrightarrow \Gamma_{0}^{\prime \prime}$, com $\Gamma_{0}^{\prime} \subseteq \Gamma_{0}$ e $\Gamma_{0}^{\prime \prime} \subseteq \Gamma_{0}$, uma função bijetora. O par $(\Gamma, \tau)$ é chamado de carcás com translação se $(\tau x)^{+}=x^{-}$, para cada $x \in \Gamma_{0}^{\prime}$.

A seguir definimos $(\mathbb{Z} \Sigma, \tau)$ que é um caso especial de carcás com translação.

Definição 1.7.2. Seja $\Sigma=\left(\Sigma_{0}, \Sigma_{1}\right)$ uma carcás conexo e acíclico. Definimos o carcás de translaçẫo infinito $(\mathbb{Z} \Sigma, \tau)$ como segue. O conjunto dos vértices de $\mathbb{Z} \Sigma$ é $\mathbb{Z} \Sigma_{0}=\mathbb{Z} \times \Sigma_{0}=\{(n, x) \mid n \in$ $\left.\mathbb{Z}, x \in \Sigma_{0}\right\}$ e para cada flecha $\alpha: x \rightarrow y$ em $\Sigma_{1}$ existem duas flechas

$$
(n, \alpha):(n, x) \rightarrow(n, y) e\left(n, \alpha^{\prime}\right):(n+1, y) \rightarrow(n, x)
$$

em $\mathbb{Z} \Sigma_{1}$, e estas são todas as flechas em $\mathbb{Z} \Sigma$.

A translação $\tau$ em $\mathbb{Z} \Sigma$ está dada por $\tau(n, x)=(n+1, x)$ para todo $(n, x) \in \mathbb{Z} \Sigma_{0}$. Para cada $(n, x) \in \mathbb{Z} \Sigma_{0}$ definimos uma bijeção entre o conjunto de flechas com final $(n, x)$ e começo $(n+1, x)$ pela fórmula

$$
\sigma(n, \alpha)=\left(n, \alpha^{\prime}\right) \text { e } \sigma\left(n, \alpha^{\prime}\right)=(n+1, \alpha)
$$

Exemplo 1.7.3. Para o carcás

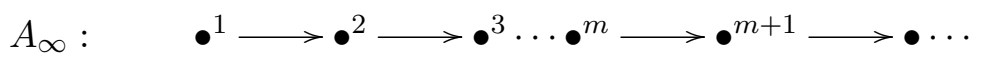

o carcás de translação $\mathbb{Z} A_{\infty}$ é da forma

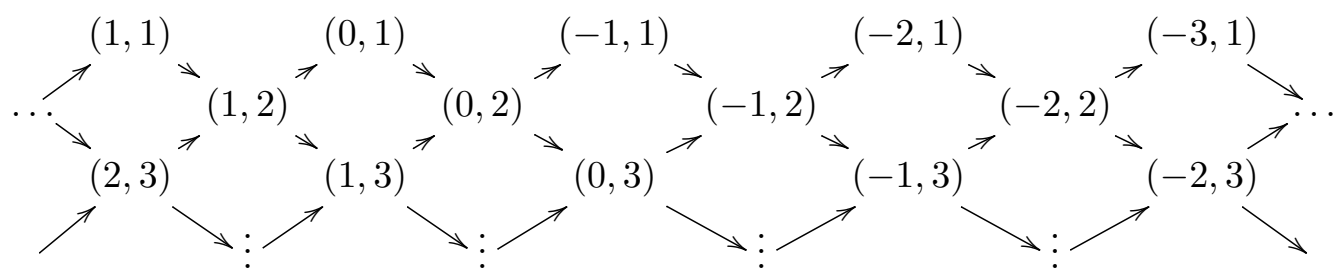

onde $\tau(n, i)=(n+1, i)$, para $n \in \mathbb{Z}$ e $i \geq 1$. Logo, por definição, $\tau$ é um automorfismo de $\mathbb{Z} A_{\infty}$, assim como suas potências $\tau^{r}$, com $r \in \mathbb{Z}$. Seja $r$ um inteiro positivo fixo e $\left(\tau^{r}\right)$ o grupo cíclico 
infinito dos automorfismos de $\mathbb{Z} A_{\infty}$ gerado por $\tau^{r}$. Denotemos por $\mathbb{Z} A_{\infty} /\left(\tau^{r}\right)$ o espaço orbital de $\mathbb{Z} A_{\infty}$ sob a ação de $\left(\tau^{r}\right)$. Assim, $\mathbb{Z} A_{\infty} /\left(\tau^{r}\right)$ é um carcás de translação, identificando cada ponto $(n, i)$ de $\mathbb{Z} A_{\infty}$ com o ponto $\tau^{r}(n, i)=(n+r, i)$, e cada flecha $\alpha: x \rightarrow y$ em $\mathbb{Z} A_{\infty}$ com a flecha $\tau^{r}: \tau^{r} x \rightarrow \tau^{r} y$. Esta construção motiva a seguinte definição.

Definição 1.7.4. Seja $(\mathcal{T}, \tau)$ um carcás com translação.

1. $(\mathcal{T}, \tau)$ é denominado um tubo estável de posto $r$ se $\mathcal{T} \cong \mathbb{Z} A_{\infty} /\left(\tau^{r}\right)$, como carcases de translação.

2. Um tubo estável $\mathcal{T}$ de posto $r=1$ é chamado um tubo homogêneo.

3. Seja $(\mathcal{T}, \tau)$ um tubo estável de posto $r \geq 1$. Uma sequência $\left(x_{1}, x_{2}, \ldots, x_{r}\right)$ de pontos em $\mathcal{T}$ é um $\tau$-ciclo $s e$

$$
\tau x_{1}=x_{r}, \tau x_{2}=x_{1}, \ldots, \tau x_{r}=x_{r-1} .
$$

4. Seja $(\mathcal{T}, \tau)$ um tubo estável. $O$ conjunto de pontos em $\mathcal{T}$ que tem exatamente um predecessor imediato (ou equivalentemente, exatamente um sucessor imediato) é chamado a boca de $\mathcal{T}$.

Exemplo 1.7.5. Um tubo estável de posto 3 é obtido do seguinte carcás

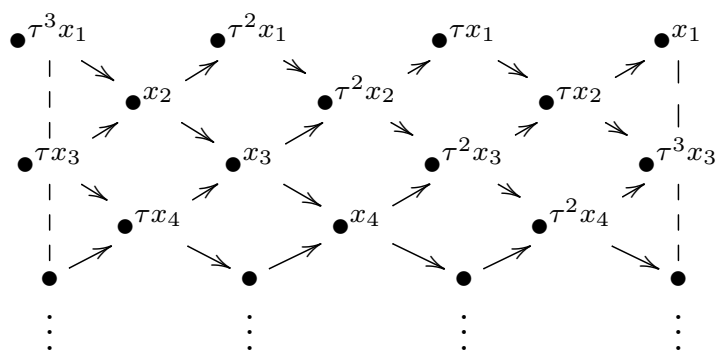

por identificação dos pontos na mesma $\tau$-órbita que estão sobre as linhas pontelhadas.

Definição 1.7.6. Seja $\mathcal{C}$ uma componente do carcás de Auslander-Reiten $\Gamma(\bmod A)$ de uma álgebra $A$, e suponhamos, por simplicidade, que $\mathcal{C}$ não tem flechas múltiplas.

1. A categoria de caminhos $K \mathcal{C}$ é a categoria definida como segue: os objetos em $K \mathcal{C}$ são os vértices em $\mathcal{C}$, e os morfismos de $x \in \mathcal{C}_{0}$ para $y \in \mathcal{C}_{0}$ são combinações $K$-lineares de caminhos em $\mathcal{C}$ de $x$ a $y$.

2. Definimos o ideal $M_{\mathcal{C}}$ na categoria $K \mathcal{C}$ como segue: cada ponto não projetivo $x \in \mathcal{C}_{0}$ 
corresponde com um mesh em $\mathcal{C}$ da forma

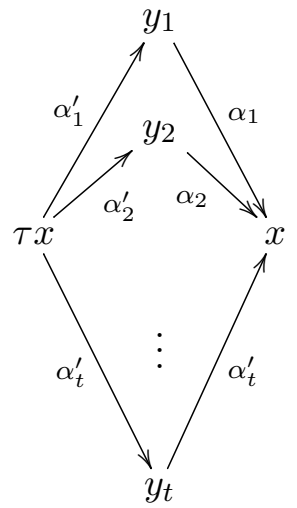

e para cada mesh associamos um elemento $m_{x}$ de $\operatorname{Hom}_{K \mathcal{C}}(\tau x, x)$, chamado elemento mesh definido pela fórmula

$$
m_{x}=\sum_{i=1}^{t} \alpha_{i}^{\prime} \alpha_{i} .
$$

Denotamos por $M_{\mathcal{C}}$ o ideal de KC gerado por todos os elementos mesh $m_{x}$, ou seja

$$
M_{\mathcal{C}}=\left\langle m_{x}: x \text { não é um vértice projetivo de } \mathcal{C}\right\rangle .
$$

3. A categoria mesh é a categoria quociente $K \mathcal{C} / M_{\mathcal{C}}$.

Definição 1.7.7. Sejam $A$ uma álgebra e $\mathcal{C}$ uma componente do carcás $\Gamma(\bmod A)$.

1. A componente $\mathcal{C}$ é chamada componente estândar de $\Gamma(\bmod A)$ se existir uma equivalência de K-categorias

$$
K \mathcal{C}=K \mathcal{C} / M_{\mathcal{C}}
$$

onde Ind $\mathcal{C}$ é a $K$-subcategoria plena de $\bmod A$ cujos objetos são representantes das classes de isomorfismos de módulos indecomponíveis em $\mathcal{C}$.

2. $\mathcal{C}$ é uma componente auto hereditária de $\Gamma(\bmod A)$ se, para cada par de A-módulos indecomponiveis $X$ e $Y$ em $\mathcal{C}$ tivermos que $\operatorname{Ext}_{A}^{2}(X, Y)=0$.

Definição 1.7.8. Sejam A uma álgebra e $\mathcal{T}$ um tubo estável do carcás de Auslander-Reiten $\Gamma(\bmod A)$. Então:

1. O tubo $\mathcal{T}$ é chamado hereditário se para cada módulo indecomponivel $X$ em $\mathcal{T}$ vale que $\operatorname{pd} X \leq 1 e$ id $X \leq 1$.

2. O tubo $\mathcal{T}$ é chamado auto-hereditário se para cada par de módulos indecomponiveis $X$ e $Y$ em $\mathcal{T}$ vale que $\operatorname{Ext}_{A}^{2}(X, Y)=0$. 
Seja $A$ uma álgebra. Um tijolo $E$ em $\bmod A$ é um módulo (necessariamente indecomponível) tal que $\operatorname{End}_{A}(E) \cong K$. Dois tijolos $E$ e $E^{\prime}$ em $\bmod A$ são ortogonais se $\operatorname{Hom}_{A}\left(E, E^{\prime}\right)=0$ e $\operatorname{Hom}_{A}\left(E^{\prime}, E\right)=0$.

Seja $E_{1}, \ldots, E_{r}$ uma família de tijolos dois a dois ortogonais em $\bmod A$. Vamos denotar por

$$
\mathcal{E}=\mathcal{E}_{A}=\mathcal{E} \mathcal{X} \mathcal{T}_{A}\left(E_{1}, \ldots, E_{r}\right)
$$

a subcategoria plena de $\bmod A$ cujos objetos não nulos são todos os $A$-módulos tais que existe uma cadeia de submódulos

$$
0 \varsubsetneqq M_{1} \varsubsetneqq M_{2} \varsubsetneqq \ldots M_{m-1} \varsubsetneqq M_{m}=M,
$$

com $m \geq 1$ e $M_{i} / M_{i-1} \cong E_{j}$, para algum $1 \leq j \leq r$.

Um objeto $S$ de $\mathcal{E}_{A}$ é chamado objeto simples se os únicos subobjetos de $S$ em $\mathcal{E}_{A}$ são o objeto nulo e o próprio $S$.

Observamos que $\mathcal{E} \mathcal{X} \mathcal{T}_{A}\left(E_{1}, \ldots, E_{r}\right)$ é a menor subcategoria aditiva contendo os $A$-módulos $E_{1}, \ldots, E_{r}$ e que é fechada por extensões.

Teorema 1.7.9. Sejam A uma álgebra e $\left\{E_{1}, \ldots, E_{r}\right\}$ uma familia finita de tijolos dois a dois ortogonais. Então:

1. (2.1 Cap X em [17]) $\mathcal{E}_{A}=\mathcal{E} \mathcal{X} \mathcal{T}_{A}\left(E_{1}, \ldots, E_{r}\right)$ é uma subcategoria abeliana, exata e fechada por extensões de $\bmod A$ e $\left\{E_{1}, \ldots, E_{r}\right\}$ é um conjunto completo de objetos dois a dois não isomorfos e simples de $\mathcal{E}_{A}$.

2. (2.6 Cap. X em [17]) Se $\left\{E_{1}, \ldots, E_{r}\right\}$ é uma familia auto-hereditária de $\bmod A$, então

(a) Todos os objetos indecomponiveis na categoria $\mathcal{E} \mathcal{X} \mathcal{T}_{A}\left(E_{1}, \ldots, E_{r}\right)$ formam uma componente auto-hereditária $\mathcal{T}_{\mathcal{E}}$ de $\Gamma(\bmod A)$.

(b) A componente $\mathcal{T}_{\mathcal{E}}$ é um tubo estável de posto $r$.

(c) Os módulos $\left\{E_{1}, \ldots, E_{r}\right\}$ formam um conjunto completo de módulos na boca do tubo $\mathcal{T}_{\mathcal{E}}$ 


\section{Capítulo 2}

\section{Sistemas estratificantes sobre álgebras hereditárias}

Os sistemas estratificantes foram introduzidos, em [7], por K. Erdmann e C. Sáenz, como uma generalização dos módulos estândares.

Em [10, 11], E. Marcos, O. Mendoza e C. Sáenz introduziram os sistemas estratificantes via módulos relativamente projetivos e os sistemas estratificantes via módulos relativamente simples, e mostraram que as três noções de sistema estratificante são equivalentes.

Neste capítulo apresentaremos as definições de sistema estratificante (ou sistema estratificante via módulos relativamente injetivos) e a de sistema estratificante via módulos relativamente simples, que serão as que usaremos ao longo deste texto. Veremos que, quando definidos sobre uma álgebra hereditária, os sistemas estratificantes estão estreitamente relacionados com os módulos inclinantes. Usando tal relação exibimos um limitante para seu tamanho. Definimos também o conjunto dos módulos estândares, as álgebras estandarmente estratificadas e, uma classe particular delas, as álgebras quase-hereditárias.

Usando o grupo de Grothendieck para um sistema estratificante, faremos uma demonstração simples de que uma álgebra estandarmente estratificada é quase-hereditária se, e somente se, tem dimensão global finita.

Veremos que, o conceito de sistema estratificante sobre uma álgebra hereditária e o de sequência excepcional são equivalentes e, usando resultados de [6], obtemos todos os sistemas estratificantes completos sobre uma álgebra de Kronecker (ou Kronecker generalizada).

Ressaltamos que, sempre que falarmos de $A$ como uma $K$-álgebra, estamos assumindo que $K$ é um corpo algebricamente fechado é que $A$ é uma $K$-álgebra associativa com unidade e de dimensão finita sobre $K$. 


\subsection{Sistemas estratificantes}

Sejam $A$ uma $K$-álgebra e $\theta=\left\{\theta_{1}, \ldots, \theta_{t}\right\}$ um conjunto finito de $A$-módulos. Denotamos por $\mathcal{F}(\theta)$ à subcategoria plena de $\bmod A$ que contem o módulo zero e todos os módulos que são filtrados por $\theta$. Isto é, um módulo não nulo $M$ está em $\mathcal{F}(\theta)$ se existir uma cadeia finita de submódulos de $M$ da forma

$$
0=M_{0} \subseteq M_{1} \subseteq \ldots \subseteq M_{n}=M,(*)
$$

e tal que o quociente $M_{i} / M_{i-1}$ é isomorfo a algum dos elementos no conjunto $\theta$, para todo $i=1,2, \ldots, n$. A cadeia (*) é chamada de $\theta$-filtração de $M$.

Com esta notação é possível enunciar a primeira das definições de sistema estratificante, que apareceu em [7], e que também é conhecida como sistema estratificante via módulos relativamente injetivos (Ver [11]).

Definição 2.1.1. [7] Sejam $K$ um corpo algebricamente fechado e $A$ uma $K$-álgebra de dimensão finita sobre $K$. Sejam $\theta=\left\{\theta_{1}, \ldots, \theta_{t}\right\}$ um conjunto fixo de A-módulos e $\underline{Y}=\left\{Y_{1}, \ldots, Y_{t}\right\}$ um conjunto de A-módulos indecomponíveis. Além disso, seja $\preceq$ uma relação de ordem total no conjunto $\{1, \ldots, t\}$. Dizemos que $(\theta, \underline{Y}, \preceq)$ é um sistema estratificante de tamanho $t$ se as seguintes condições são satisfeitas:

1. $\operatorname{Hom}_{A}\left(\theta_{j}, \theta_{i}\right)=0$, para $j \succ i$.

2. Para cada $i=1, \ldots, t$, existe uma sequência exata

$$
0 \longrightarrow \theta_{i} \stackrel{\alpha_{i}}{\longrightarrow} Y_{i} \longrightarrow Z_{i} \longrightarrow 0
$$

onde $Z_{i}$ é filtrado por $\left\{\theta_{j}\right\}_{j \prec i}$.

3. $\left.\operatorname{Ext}_{A}^{1}\left(\_, Y\right)\right|_{\mathcal{F}(\theta)}=0$, onde $Y=\oplus_{i=1}^{t} Y_{i}$.

Da definição acima, temos que $\theta_{1} \cong Y_{1}$ e que (para $t \geq 2$ ) existe uma sequência exata da forma

$$
0 \longrightarrow \theta_{2} \stackrel{\alpha_{2}}{\longrightarrow} Y_{2} \longrightarrow Y_{1}^{m} \longrightarrow 0, \text { com } m \geq 0 .
$$

A seguinte proposição permite uma caracterização dos sistemas estratificantes que depende somente dos elementos no conjunto $\theta$.

Proposição 2.1.2. [7] Sejam $K$ um corpo algebricamente fechado, A uma K-álgebra de dimensão finita sobre $K, \preceq$ uma relação de ordem total no conjunto $\{1, \ldots, t\}$ e $\theta=\left\{\theta_{1}, \ldots, \theta_{t}\right\}$ um conjunto de A-módulos. Então as condições são equivalentes:

1. Existe um conjunto de A-módulos $\underline{Y}=\left\{Y_{1}, \ldots, Y_{t}\right\}$ tal que $(\theta, \underline{Y}, \preceq)$ é um sistema estratificante de tamanho $t$. 
2. O conjunto $\theta$ satisfaz as condições a seguir:

(a) Os elementos de $\theta$ são A-módulos indecomponíveis.

(b) $\operatorname{Hom}_{A}\left(\theta_{j}, \theta_{i}\right)=0$, para $j \succ i$.

(c) $\operatorname{Ext}_{A}^{1}\left(\theta_{j}, \theta_{i}\right)=0$, para $j \succeq i$.

Fazendo uso da caracterização acima, em [11], os autores introduziram a seguinte definição de sistema estratificante.

Definição 2.1.3. [11] Sejam $K$ um corpo algebricamente fechado, $A$ uma $K$-álgebra de dimensão finita sobre $K, \preceq$ uma relação de ordem total no conjunto $\{1, \ldots, t\}$ e $\theta=\left\{\theta_{1}, \ldots, \theta_{t}\right\}$ um conjunto de A-módulos indecomponiveis. Dizemos que o par $(\theta, \preceq)$ é um sistema estratificante de tamanho $t$ se as seguintes condições são satisfeitas:

1. $\operatorname{Hom}_{A}\left(\theta_{j}, \theta_{i}\right)=0$, para $j \succ i$.

2. $\operatorname{Ext}_{A}^{1}\left(\theta_{j}, \theta_{i}\right)=0, \operatorname{para} j \succeq i$.

Esta definição também aparece na literatura como sistema estratificante via módulos relativamente simples (Ver [11]).

Tendo em vista a anterior proposição usaremos indistintamente uma ou outra definição de sistema estratificante. Além disso, dado que por simplicidade usaremos a relação de ordem natural dos números inteiros, não será feita mais referência a tal relação de ordem.

Sejam $\theta=\left\{\theta_{1}, \ldots, \theta_{t}\right\}$ um sistema estratificante, $M$ um $A$-módulo qualquer e

$$
0=M_{0} \subseteq M_{1} \subseteq \ldots \subseteq M_{n}=M
$$

uma $\theta$ filtração $M$. Sabemos que o número de vezes que $\theta_{i}$ aparece numa $\theta$ filtração de $M$ independe da $\theta$-filtração (ver [7]). Tal número é conhecido como a multiplicidade de $\theta_{i}$ em uma $\theta$-filtração de $M$ e o denotamos por $\left[M: \theta_{i}\right]$. Através da multiplicidade de $\theta_{i}$ definimos o $\theta$ comprimento de $M$, que denotamos por $\ell_{\theta}(M)$, que é

$$
\ell_{\theta}(M)=\sum_{i=1}^{t}\left[M: \theta_{i}\right] .
$$

Os elementos no conjunto $\theta$ são chamados de elementos relativamente simples da categoria $\mathcal{F}(\theta)$, uma vez que não admitem submódulos próprios na categoria.

Por outro lado se $A_{A} \in \mathcal{F}(\theta)$, ou equivalentemente se todos os projetivos indecomponíveis estão em $\mathcal{F}(\theta)$, dizemos que $\theta$ é um sistema estratificante estândar.

Se $\theta$ é um sistema estratificante, $\mathcal{F}(\theta)$ é fechada por extensões e, portanto, por somas diretas. Mais ainda, $\mathcal{F}(\theta)$ é fechada por somandos diretos e funtorialmente finita (Ver [11]) e, portanto, tem sequências de Auslander-Reiten relativas (Ver [15]). No entanto, em geral, ela não é fechada por núcleos de epimorfismos como veremos no seguinte exemplo. 
Exemplo 2.1.4. (em [11]) Seja $A \cong K Q$, onde $Q$ é o carcás $1 \longrightarrow 2$. Então o conjunto $\theta=$ $\left\{\theta_{1}=I_{2}, \theta_{2}=I_{1}\right\}$ é um sistema estratificante. Consideremos a seguinte sequência exata

$$
0 \longrightarrow S_{2} \longrightarrow I_{2} \longrightarrow I_{1} \longrightarrow 0
$$

Como $P_{2}=S_{2} \notin \mathcal{F}(\theta)$ a categoria $\mathcal{F}(\theta)$ não é fechada por núcleos de epimorfismos e $\theta$ não é um sistema estratificante estândar.

Definição 2.1.5. Sejam $\mathcal{C}$ uma subcategoria plena de $\bmod A$ e $X$ um objeto em $\mathcal{C}$. Dizemos que $X$ é relativamente projetivo na categoria $\mathcal{C}$ se $\left.\operatorname{Ext}_{A}^{1}(X,-)\right|_{\mathcal{C}}=0$. Dualmente, dizemos que $X$ é relativamente injetivo em $\mathcal{C}$ se vale a igualdade $\left.\operatorname{Ext}_{A}^{1}(\ldots, X)\right|_{\mathcal{C}}=0$.

Dado um sistema estratificante $(\theta, \underline{Y})$ sobre a $K$-álgebra $A$, denotamos por $\mathcal{Y}(\theta)$ a subcategoria plena dos módulos $X \in \bmod A$ tais que $\left.\operatorname{Ext}_{A}^{1}(\ldots, X)\right|_{\mathcal{F}(\theta)}=0$. Em outras palavras, temos que

$$
\mathcal{Y}(\theta)=\left\{X \in \bmod A: \operatorname{Ext}_{A}^{1}\left(X, \theta_{i}\right)=0, \text { para todo } i=1, \ldots, t\right\} .
$$

Destacamos que os elementos do conjunto $\mathcal{F}(\theta) \cap \mathcal{Y}(\theta)$ são $A$-módulos relativamente injetivos a categoria $\mathcal{F}(\theta)$. O teorema abaixo fornece uma caracterização de tais módulos.

Teorema 2.1.6. [11] Seja $(\theta, \underline{Y})$ um sistema estratificante de tamanho t. Então

$$
\mathcal{F}(\theta) \cap \mathcal{Y}(\theta)=\operatorname{add} Y \text {, onde } Y=\bigoplus_{i=1}^{t} Y_{i} .
$$

A seguir definiremos o grupo de Grothendieck para um sistema estratificante e veremos que, assim como o grupo de Grothendieck de uma álgebra, é um grupo livre gerado por um conjunto finito.

Definição 2.1.7. Seja $\theta$ um sistema estratificante de tamanho t. $O$ grupo de Grothendieck de $\theta$ é o grupo abeliano $K_{0}(\theta)=F_{\theta} / F_{\theta}^{\prime}$, onde $F_{\theta}$ é o grupo abeliano livre que cuja base é o conjunto de classes de isomorfismos $\widetilde{M}$ de módulos $M$ em $\mathcal{F}(\theta)$ e $F_{\theta}^{\prime}$ é o subgrupo de $F_{\theta}$ gerado pelos elementos da forma $\widetilde{M}-\widetilde{L}-\widetilde{N}$ correspondentes às sequências exatas $0 \rightarrow L \rightarrow M \rightarrow N \rightarrow 0$ em $\mathcal{F}(\theta)$.

Denotamos por $[M]$ a imagem da classe de isomorfismo $\widetilde{M}$ do módulo $M$ pelo epimorfismo canônico de grupos $\pi: F_{\theta} \longrightarrow F_{\theta} / F_{\theta}^{\prime}$. Definimos o homomorfismo de grupos $\underline{\operatorname{dim}}_{\theta}: K_{0}(\theta) \longrightarrow \mathbb{Z}^{t}$ da seguinte forma 


$$
\underline{\operatorname{dim}}_{\theta}([M])=\left[\begin{array}{c}
{\left[M: \theta_{1}\right]} \\
\vdots \\
{\left[M: \theta_{t}\right]}
\end{array}\right]
$$

Observação 2.1.8. Se $0 \longrightarrow N \longrightarrow M \longrightarrow L \longrightarrow 0$ é uma sequência exata curta de $A$-módulos em $\mathcal{F}(\theta)$, então

$$
\underline{\operatorname{dim}}_{\theta}([M])=\underline{\operatorname{dim}}_{\theta}([N])+\underline{\operatorname{dim}}_{\theta}([L]) .
$$

Proposição 2.1.9. Seja $\theta$ um sistema estratificante de tamanho t. Então o grupo de Grothendieck de $\theta, K_{0}(\theta)$, é um grupo abeliano livre que tem como base o conjunto $\left\{\left[\theta_{1}\right], \ldots,\left[\theta_{t}\right]\right\}$. Além disso, o homomorfismo de grupos $\underline{\operatorname{dim}}_{\theta}$ é um isomorfismo de grupos.

Demonstração. Sejam $M \in \mathcal{F}(\theta)$ e $0=M_{0} \subseteq M_{1} \subseteq M_{2} \subseteq \ldots \subseteq M_{\ell}=M$ uma $\theta$-filtração de $M$. Pela definição de $K_{0}(\theta)$, temos que

$$
\begin{aligned}
{[M] } & =\left[M_{t} / M_{t-1}\right]+\left[M_{t-1}\right] \\
& =\sum_{j=1}^{\ell}\left[M_{j} / M_{j-1}\right] \\
& =\sum_{j=1}^{\ell}\left[M: \theta_{j}\right]\left[\theta_{j}\right] .
\end{aligned}
$$

Isto prova que $\left\{\left[\theta_{1}\right], \ldots,\left[\theta_{t}\right]\right\}$ gera o grupo $K_{0}(\theta)$. Por outro lado, é claro que $M \cong N$ implica que $\underline{\operatorname{dim}}_{\theta}([M])=\underline{\operatorname{dim}}_{\theta}([N])$. Desde que a imagem pelo homomorfismo $\underline{\operatorname{dim}}_{\theta}$ do conjunto gerado por $\left\{\left[\theta_{1}\right], \ldots,\left[\theta_{t}\right]\right\}$ é a base canônica do grupo livre $\mathbb{Z}^{t}$, então o conjunto $\left\{\left[\theta_{1}\right], \ldots,\left[\theta_{t}\right]\right\}$ é $\mathbb{Z}$ linearmente independente em $K_{0}(\theta)$.

Portanto, $K_{0}(\theta)$ é o grupo livre gerado por $\left\{\left[\theta_{1}\right], \ldots,\left[\theta_{t}\right]\right\}$ e o homomorfismo $\underline{\operatorname{dim}}_{\theta}: K_{0}(\theta) \rightarrow \mathbb{Z}^{t}$ é um isomorfismo de grupos.

\subsection{Módulos estândares}

Definiremos o conjunto dos módulos estândares, as álgebras estandarmente estratificadas e uma classe particular delas: as álgebras quase-hereditárias. Além disso, usando o grupo de Grothendieck para um sistema estratificante, faremos uma demonstração simples de que uma álgebra estandarmente estratificada é quase-hereditária se, e somente se, tem dimensão global finita.

Mais detalhes acerca dos tópicos tratados aqui podem ser encontrados, por exemplo, em $[15,21,5]$.

Definição 2.2.1. Dados os A-módulos $X$ e $Y$, definimos o traço de $Y$ em $X$, que denotamos por $\tau_{Y}(X)$, como o submódulo de $X$ gerado pelas imagens dos homomorfismos de $Y$ em $X$, isto $\dot{e}$,

$$
\tau_{Y}(X)=\left\langle\operatorname{Im} \varphi: \varphi \in \operatorname{Hom}_{A}(Y, X)\right\rangle .
$$


Seja $A$ uma $K$-álgebra. Dado um conjunto completo de idempotentes ortogonais e primitivos $\left\{e_{1}, \ldots, e_{n}\right\}$ de $A$, para cada $i, 1 \leq i \leq n$, denotamos por $\varepsilon_{i}$ o idempotente

$$
\varepsilon_{i}=e_{i}+e_{i+1}+\cdots+e_{n}
$$

e definimos $\varepsilon_{n+1}=0$. Por fim, usando esta notação, definimos os módulos estândares.

Definição 2.2.2. Seja $\boldsymbol{e}=\left(e_{1}, \ldots, e_{n}\right)$ uma ordem fixada de um conjunto ordenado e completo de idempotentes ortogonais e primitivos $\left\{e_{1}, \ldots, e_{n}\right\}$ de A. A sequência $\Delta=\left(\triangle_{1}, \ldots, \triangle_{n}\right)$ de módulos estândares à direita, com respeito à ordem e, é dada por

$$
\triangle_{i}=P_{i} / \tau_{\varepsilon_{i+1} A}\left(P_{i}\right)=P_{i} / e_{i} A \varepsilon_{i+1} A
$$

De forma equivalente, o módulo estândar $\triangle_{i}$ define-se como o quociente maximal de $P_{i}$ tal que se $\left[\triangle_{i}: S_{j}\right] \neq 0$ então $j \leq i$.

\section{Proposição 2.2.3. (Propriedades dos módulos estândares)}

1. $\operatorname{Hom}_{A}\left(\triangle_{i}, \triangle_{j}\right)=0$, se $j<i$.

2. $\operatorname{Ext}_{A}^{1}\left(\triangle_{i}, \triangle_{j}\right)=0$, se $j \leq i$.

Como os módulos estândares têm topo simples, então eles são indecomponíveis. Em vista disto o conjunto $\triangle$ é um sistema estratificante de tamanho o posto de $K_{0}(A)$.

Definição 2.2.4. Sejam A uma $K$-álgebra, $\triangle$ a sequência dos módulos estândares relativa a uma ordem $\boldsymbol{e}=\left(e_{1}, \ldots, e_{n}\right)$ fixada dos idempotentes. Dizemos que $A$ é uma álgebra estandarmente estratificada se $A_{A} \in \mathcal{F}(\triangle)$.

De forma equivalente, $A$ é uma álgebra estandarmente estratificada se todos os módulos projetivos indecompiníveis são filtrados por $\triangle$.

O próximo exemplo mostra que a propriedade de uma álgebra ser estandarmente estratificada depende da ordem fixada para os idempotentes.

Exemplo 2.2.5. Seja $A \cong k Q / I$ onde $I=\left\langle\alpha \beta, \beta^{2}\right\rangle$ e $Q$ é o carcás

$$
1 \cdot \stackrel{\alpha}{\longrightarrow} \cdot 2 \bigcirc \beta
$$

Na ordem $\boldsymbol{e}_{\mathbf{1}}=\left(e_{1}, e_{2}\right)$ o conjunto dos módulos estândares é $\triangle=\left\{\triangle_{1}=S_{1}, \triangle_{2}=P_{2}\right\}$. Logo, $A$ junto com $\boldsymbol{e}_{\mathbf{1}}$ não é estandarmente estratificada, pois a única cadeia de submódulos de $P_{1}$ é

$$
0 \subset \operatorname{rad} P_{1}=S_{2} \subset P_{1},
$$

que não é uma $\triangle$-filtração uma vez que $S_{2} / 0 \cong S_{2} \notin \triangle$. No entanto, na ordem $e_{2}=\left(e_{2}, e_{1}\right)$ o conjunto dos módulos estândares é $\triangle=\left\{\triangle_{1}=P_{2}, \triangle_{2}=P_{1}\right\}$. Isto é $A$ junto com $e_{2}$ é uma álgebra estandarmente estratificada. 
As álgebras estandarmente estratificadas têm muitas propriedades interessantes das quais enunciaremos algumas. Mas antes definiremos módulo inclinante generalizado.

Definição 2.2.6. Seja A uma K-álgebra. Um A-módulo $T$ é dito módulo inclinante generalizado se satisfaz as condiçôes abaixo:

1. $\operatorname{pd} T<\infty$.

2. $\operatorname{Ext}_{A}^{i}(T, T)=0$, para todo inteiro $i \geq 1$.

3. Existe uma sequência exata do tipo

$$
0 \longrightarrow A_{A} \longrightarrow T_{0} \longrightarrow T_{1} \longrightarrow \cdots \longrightarrow T_{s} \longrightarrow 0
$$

com $T_{i} \in$ add $T$, para $i=0,1, \ldots, s$.

Teorema 2.2.7. [21] Seja A uma K-álgebra estandarmente estratificada relativa à sequência de A-módulos (à direita) estândares $\triangle=\left(\triangle_{1}, \ldots, \triangle_{n}\right)$. Então:

1. Os módulos relativamente projetivos em $\mathcal{F}(\triangle)$ são justamente os $A$-módulos projetivos.

2. $\mathcal{F}(\triangle)$ é fechada por núcleos de epimorfismos.

3. Existe um A-módulo inclinante generalizado $T$, único a menos da multiplicidade dos somandos diretos indecomponíveis, tal que

$$
\text { add } T=\mathcal{F}(\triangle) \cap \mathcal{Y}(\triangle) \text {. }
$$

4. A álgebra $B=\operatorname{End}_{A}(T)$ é estandarmente estratificada com sequência de B-módulos (à esquerda) estândares $\triangle^{\prime}=\left(\triangle_{1}^{\prime}, \ldots, \triangle_{n}^{\prime}\right)$, onde $\triangle_{i}^{\prime}=\operatorname{Hom}_{A}\left(\triangle_{n-i+1}, T\right)$.

Definição 2.2.8. Dizemos que $A$ é uma álgebra quase-hereditária se $A$ é uma álgebra estandarmente estratificada e $\operatorname{End}_{A}\left(\triangle_{i}\right)$ é um anel com divisão para $i=1,2, \ldots, n$, onde $n=$ posto $K_{0}(A)$

Equivalentemente, $A$ é quase-hereditária se, e somente se, $A$ é estandarmente estratificada e $\left[\triangle_{i}: S_{i}\right]=1$, para $i=1, \ldots, n$.

Exemplo 2.2.9. 1. Uma álgebra hereditária é quase-hereditária com relação a qualquer ordem $\boldsymbol{e}$ de um conjunto completo de idempotentes ortogonais e primitivos. Mais ainda, se $A$ é uma álgebra quase-hereditária com relação a qualquer ordem $\boldsymbol{e}$ de um conjunto completo de idempotentes primitivos e ortogonais, então $A$ é uma álgebra hereditária [8]. 
2. Seja $A$ a álgebra de caminhos do seguinte carcás

$$
\dot{1} \stackrel{\alpha_{1}}{\longrightarrow} \underset{2}{\stackrel{\alpha_{2}}{\longrightarrow}} \dot{3} \longrightarrow \cdots \longrightarrow \underset{n-1}{\cdot} \stackrel{\alpha_{n}}{\longrightarrow} \cdot
$$

limitado pelas relações $\alpha_{i} \alpha_{i-1}=0$, para $i=2, \ldots, n$. Com relação à ordem $\boldsymbol{e}=\left(e_{1}, \ldots, e_{n}\right)$ temos que $\triangle_{i}=S_{i}$ e, portanto, $A$ é uma álgebra quase-hereditária.

Observação 2.2.10. Como, pela Proposição 1.5.4, o homomorfismo de grupos $\underline{\operatorname{dim}}: K_{0}(A) \rightarrow \mathbb{Z}^{n}$, definido por

$$
\underline{\operatorname{dim}}([M])=\left[\begin{array}{c}
{\left[M: S_{1}\right]} \\
\vdots \\
{\left[M: S_{n}\right]}
\end{array}\right]
$$

é um isomorfismo, pode-se considerar a composta $(\underline{\operatorname{dim}})^{-1} \underline{\operatorname{dim}}_{\triangle}: K_{0}(\triangle) \longrightarrow K_{0}(A)$. Se escrevemos a matriz de tal homomorfismo relativo às bases $\left\{\left[\triangle_{i}\right]\right\}_{i=1}^{n}$ e $\left\{\left[S_{i}\right]\right\}_{i=1}^{n}$ para $K_{0}(\triangle)$ e $K_{0}(A)$, respectivamente, obtemos

$$
D=\left[\begin{array}{cccc}
{\left[\triangle_{1}: S_{1}\right]} & {\left[\triangle_{2}: S_{1}\right]} & \ldots & {\left[\triangle_{n}: S_{1}\right]} \\
{\left[\triangle_{1}: S_{2}\right]} & {\left[\triangle_{2}: S_{2}\right]} & \ldots & {\left[\triangle_{n}: S_{2}\right]} \\
{\left[\triangle_{1}: S_{3}\right]} & {\left[\triangle_{2}: S_{3}\right]} & \ldots & {\left[\triangle_{n}: S_{3}\right]} \\
\vdots & \vdots & & \vdots \\
{\left[\triangle_{1}: S_{n}\right]} & {\left[\triangle_{2}: S_{n}\right]} & \ldots & {\left[\triangle_{n}: S_{n}\right]}
\end{array}\right]
$$

Como os fatores de composição do módulo $\triangle_{i}$ são módulos simples $S_{j}$, com $j \leq i$, então a matriz $D$ tem a forma

$$
D=\left[\begin{array}{cccc}
{\left[\triangle_{1}: S_{1}\right]} & {\left[\triangle_{2}: S_{1}\right]} & \ldots & {\left[\triangle_{n}: S_{1}\right]} \\
0 & {\left[\triangle_{2}: S_{2}\right]} & \ldots & {\left[\triangle_{n}: S_{2}\right]} \\
0 & 0 & \ldots & {\left[\triangle_{n}: S_{3}\right]} \\
\vdots & \vdots & & \vdots \\
0 & 0 & \ldots & {\left[\triangle_{n}: S_{n}\right]}
\end{array}\right] .
$$

Portanto det $D=\left[\triangle_{1}: S_{1}\right]\left[\triangle_{2}: S_{2}\right] \cdots\left[\triangle_{n}: S_{n}\right]>0$.

Como consequência temos o seguinte corolário.

Corolário 2.2.11. Se A é uma K-álgebra estandarmente estratificada, então A é uma álgebra quase-hereditária se, e somente se, $\operatorname{det} D=1$.

Demonstração. Decorre imediatamente da anterior observação e da definição de álgebra quasehereditária. 
Agora suponhamos que $A$ é uma álgebra estandarmente estratificada. Neste caso, se $X \in$ $\mathcal{F}(\triangle)$ então pd $X \leq n-1$ (Ver [1]). Isto implica que o conjunto $\left\{\left[P_{i}\right]\right\}_{i=1}^{n}$ é uma base para $K_{0}(\triangle)$. Usando esta base para $K_{0}(\triangle)$ podemos escrever a seguinte matriz para o homomorfismo $(\underline{\operatorname{dim}})^{-1} \underline{\operatorname{dim}} \triangle$

$$
C=\left[\begin{array}{cccc}
{\left[P_{1}: S_{1}\right]} & {\left[P_{2}: S_{1}\right]} & \ldots & {\left[P_{n}: S_{1}\right]} \\
{\left[P_{1}: S_{2}\right]} & {\left[P_{2}: S_{2}\right]} & \ldots & {\left[P_{n}: S_{2}\right]} \\
{\left[P_{1}: S_{3}\right]} & {\left[P_{2}: S_{3}\right]} & \ldots & {\left[P_{n}: S_{3}\right]} \\
\vdots & \vdots & & \vdots \\
{\left[P_{1}: S_{n}\right]} & {\left[P_{2}: S_{n}\right]} & \ldots & {\left[P_{n}: S_{n}\right]}
\end{array}\right]
$$

Deste modo $C$ e $D$ são matrizes semelhantes e, portanto, $\operatorname{det} D=\operatorname{det} C$. Por outro lado, notemos que $C$ é justamente a matriz de Cartan de $A$. Portanto temos o seguinte resultado.

Corolário 2.2.12. Seja A uma álgebra estandarmente estratificada. Então o determinante da matriz de Cartan de A é positivo.

Proposição 2.2.13. Seja A uma álgebra estandarmente estratificada com dimensão global finita. Então A é uma álgebra quase-hereditária.

Demonstração. Se a dimensão global de $A$ é finita então, segundo a Proposição 3.10 do Cap. III em [2], $\operatorname{det} C \in\{-1,1\}$. De outro lado, como $\operatorname{det} C>0$, segundo o corolário acima, então $\operatorname{det} C=1$. Portanto, como consequência do Corolário 2.2.11, $A$ é uma álgebra quase-hereditária.

Proposição 2.2.14. [8] Se A uma álgebra quase-hereditária então:

1. pd $\triangle_{i} \leq n-i$.

2. $\operatorname{pd} S_{i} \leq n+i-2$.

3. $\operatorname{gd} A \leq 2(n-1)$.

Finalmente, usando a proposição acima e o Corolário 2.2.13, temos o seguinte teorema.

Teorema 2.2.15. Seja A uma K-álgebra estandarmente estratificada. Então A é quase-hereditária se, e somente se, a dimensão global de A é finita.

\subsection{Sequências excepcionais}

O conceito de sequência excepcional foi introduzido por Gordentsev e Rudakov em [9]. Tais sequências têm sido usadas para estudar fibrados vetoriais sobre $\mathbb{P}^{2}$ e categorias derivadas de 
variedades algébricas (ver por exemplo $[4,16]$ ). Além disso, Crawely-Boevey considerou a noção correspondente no contexto das representações de um carcás e, em [6], demonstrou que se $K$ é um corpo algebricamente fechado e $A$ é uma $K$-álgebra hereditária, existe uma ação transitiva do grupo de tranças gerado por $s-1$ elementos sobre o conjunto de sequências excepcionais completas de tamanho $s$. Depois Ringel, em [14], mostrou que o mesmo resultado vale se $A$ for uma álgebra de Artin.

Em [20], U. Seidel calculou o número de sequências excepcionais completas sobre as álgebras hereditárias de tipo Dynkin. Mas a descrição concreta de sequências excepcionais não tem sido estudada para álgebras hereditárias de tipo de representação infinito.

Nesta seção mostramos que o conceito de sequência excepcional e o de sistema estratificante sobre uma álgebra hereditária coincidem. Além disso, encontramos um limitante para o tamanho de um sistema estratificante sobre uma álgebra hereditária e vemos como estes sistemas estão estreitamente relacionados com os módulos inclinantes. Também, usamos resultados de [6], para calcular todos os sistemas estratificantes completos sobre a álgebra de Kronecker e sobre as álgebras de Kronecker generalizadas.

Definição 2.3.1. Sejam $K$ um corpo algebricamente fechado e $A$ uma $K$-álgebra hereditária. Dizemos que um A-módulo $X$ é excepcional se satisfaz as seguintes condições:

1. $\operatorname{End}_{A}(X) \cong K$,

2. $\operatorname{Ext}_{A}^{1}(X, X)=0$.

Uma sequência excepcional $E=\left(X_{1}, \ldots, X_{t}\right)$ de tamanho $t$ é uma sequência de A-módulos tais que:

1. $X_{i}$ é um módulo excepcional, para $1 \leq i \leq t$.

2. $\operatorname{Hom}_{A}\left(X_{i}, X_{j}\right)=0$, para $i>j$.

3. $\operatorname{Ext}_{A}^{1}\left(X_{i}, X_{j}\right)=0$, para $i \geq j$.

Destacamos que a definição acima é bem parecida à de sistema estratificante. De fato, se os elementos de uma dada sequência excepcional são módulos indecomponíveis, então tal sequência é um sistema estratificante.

A seguir, no intuito de usar livremente os resultados sobre sequências excepcionais obtidos em [6], veremos que os conceitos de sequência excepcional e sistema estratificante sobre uma álgebra hereditária coincidem. Para isto, enunciaremos o seguinte lema que será de grande utilidade.

Lema 2.3.2. (em [2], Cap.VIII, 3.3) Sejam K um corpo algebricamente fechado e A uma $K$-álgebra hereditária de dimensão finita. Se $T_{1}$ e $T_{2}$ são A-módulos indecomponíveis tais que $\operatorname{Ext}_{A}^{1}\left(T_{2}, T_{1}\right)=0$, então qualquer homomorfismo não nulo $f: T_{1} \longrightarrow T_{2}$ é ou um monomorfismo 
ou um epimorfismo. Em particular, se $T_{1}$ é um módulo indecomponivel e $\operatorname{Ext}_{A}^{1}\left(T_{1}, T_{1}\right)=0$, então $\operatorname{End}_{A}\left(T_{1}\right) \cong K$.

Como uma consequência imediata temos o seguinte corolário.

Corolário 2.3.3. Sejam $K$ um corpo algebricamente fechado, $A$ uma $K$-álgebra de dimensão finita e hereditária. Seja $M$ um A-módulo tal que $\operatorname{Ext}_{A}^{1}(M, M)=0$. Então $M$ é indecomponível se, e somente se, $\operatorname{End}_{A}(M) \cong K$.

Demonstração. Suponhamos que $M$ é indecomponível e $\operatorname{Ext}_{A}^{1}(M, M)=0$. Então, pelo lema anterior, resulta que $\operatorname{End}_{A}(M) \cong K$. Reciprocamente, se $\operatorname{End}_{A}(M) \cong K$, então $\operatorname{End}_{A}(M)$ é um anel local e, portanto, $M$ é indecomponível.

Corolário 2.3.4. Sejam K um corpo algebricamente fechado e $A$ uma $K$-álgebra de dimensão finita. Se $A$ é hereditária, então a sequência de A-módulos $\left(X_{1}, X_{2}, \ldots, X_{t}\right)$ é uma sequência excepcional se, e somente se, o conjunto $\left\{X_{1}, X_{2}, \ldots, X_{t}\right\}$ é um sistema estratificante sobre $A$.

Antes de enunciar o próximo resultado vamos lembrar as definições de módulo inclinante parcial e de módulo inclinante.

Definição 2.3.5. Seja A uma K-álgebra. Um A-módulo $T$ é dito módulo inclinante parcial se satisfaz as seguintes condições:

(T1) pd $T \leq 1$.

(T2) $\operatorname{Ext}_{A}^{1}(T, T)=0$.

Além disso, um módulo inclinante parcial é dito módulo inclinante se existir uma sequência exata da forma

(T3) $0 \longrightarrow A_{A} \longrightarrow T_{0} \longrightarrow T_{1} \longrightarrow 0$, com $T_{i} \in$ add $T$, para $i=0,1$.

Se $T$ é um módulo inclinante parcial livre de multiplicidade no número de somandos diretos indecomponíveis dizemos que $T$ é um módulo inclinante parcial básico.

Lema 2.3.6. (3.4 Cap. VI em [2]) Sejam A uma K-álgebra e T um A-módulo inclinante parcial. Então existe um A-módulo E tal que $T \oplus E$ é um A-módulo inclinante.

A proposição a seguir da um critério para saber quando um módulo inclinante parcial $T$ é um módulo inclinante em termos do número de somando diretos indecomponíveis não isomorfos de $T$. 
Proposição 2.3.7. (4.4 Cap. VI em [2]) Sejam A uma K-álgebra e T um A-módulo inclinante parcial. Então $T$ é um módulo inclinante se, e somente se, o número de somandos diretos indecomponiveis e dois a dois não isomorfos de $T$ é igual ao posto de $K_{0}(A)$.

Do Lema 2.3.6 e proposição acima podemos afirmar que se $T$ é um $A$-módulo inclinante parcial básico então o número de somandos diretos não isomorfos de $T$ é no maximo $n=K_{0}(A)$. Como consequência temos um limitante para o tamanho de um sistema estratificante sobre uma álgebra hereditária.

Lema 2.3.8. Sejam $A=K Q$, onde $Q$ é um carcás acíclico com n vértices, e $X=\left(X_{1}, \ldots, X_{t}\right)$ um sistema estratificante sobre A. Então

$$
\mathcal{F}(X) \cap \mathcal{Y}(X)=\operatorname{add} T
$$

onde $T$ é um A-módulo inclinante parcial básico, e $t \leq n$.

Demonstração. Pelo Teorema 2.1.6 sabemos que $\mathcal{F}(X) \cap \mathcal{Y}(X)=$ add $Y$, onde $Y$ é um $A$ módulo com $t$ somandos diretos indecomponíveis dois a dois não isomorfos e tal que $\operatorname{Ext}_{A}^{1}(Y, Y)=$ 0 . Isto é, $Y$ é um módulo inclinante parcial, o que implica que $t \leq n$.

A hipótese de que $A$ seja uma álgebra hereditária no anterior lema é essencial. Para verificar isto temos o seguinte exemplo.

Exemplo 2.3.9. [11] Seja $A \cong K Q / I$, onde $Q$ é o seguinte carcás

$$
3 \stackrel{\alpha}{\longrightarrow} 1 \stackrel{\beta}{\longleftarrow} 2 \stackrel{\gamma}{\longleftarrow} 4
$$

e $I=\langle\gamma \beta\rangle$. Fazendo $\theta_{1}=S_{1}=P_{1}, \theta_{2}=P_{2}, \theta_{3}=P_{3}, \theta_{4}=P_{4}=I_{2}$ e $\theta_{5}=S_{4}$ temos que o sistema estratificante $\theta=\left\{\theta_{1}, \theta_{2}, \theta_{3}, \theta_{4}, \theta_{5}\right\}$ é estândar e de tamanho 5 .

O limitante para o tamanho de um sistema estratificante sobre uma álgebra hereditária exibido no Lema 2.3.8 incentiva a seguinte definição.

Definição 2.3.10. Sejam $Q$ um carcás acíclico com $n$ vértices e $A=K Q$. Dizemos que um sistema estratificante $X=\left(X_{1}, \ldots, X_{t}\right)$ sobre $A$ é completo se tem tamanho $n$.

Observação 2.3.11. Afirmamos que, se $\theta$ é um sistema estratificante estândar sobre uma álgebra hereditária $A=K Q$, então $\theta$ é completo. De fato, se $t$ é o tamanho de $\theta$ então, pelo Lema 2.3.8, temos que $t \leq\left|Q_{0}\right|$. De outro lado, em [11], foi demonstrado que se $\theta$ é um sistema estratificante estândar então $\left|Q_{0}\right| \leq t$. 
Mas a recíproca desta afirmação não é verdadeira, isto é, nem todo sistema estratificante completo é estândar, como mostra o Exemplo 2.1.4.

Definição 2.3.12. Sejam $(\theta, \underline{Y})$ e $\left(\theta^{\prime}, \underline{Y}^{\prime}\right)$ dois sistemas estratificantes de tamanho $t, \alpha_{i}$ : $\theta_{i} \longrightarrow Y_{i}$ e $\alpha_{i}^{\prime}: \theta_{i}^{\prime} \longrightarrow Y_{i}^{\prime}$, com $i=1, \ldots, t$, morfismos como na Definição 2.1.1. Um morfismo de sistemas estratificantes, $f:(\theta, \underline{Y}) \longrightarrow\left(\theta^{\prime}, \underline{Y}^{\prime}\right)$, é um conjunto de morfismos $f=\left\{f_{1}(i), f_{2}(i)\right\}_{i=1}^{t}$, onde $f_{1}(i): \theta_{i} \longrightarrow \theta_{i}^{\prime}$ e $f_{2}(i): Y_{i} \longrightarrow Y_{i}^{\prime}$ são homomorfismos tais que

$$
f_{2}(i) \alpha_{i}=\alpha^{\prime}(i) f_{1}(i), \text { para todo } i=1, \ldots, t \text {. }
$$

0 morfismo $f=\left\{f_{1}(i), f_{2}(i)\right\}_{i=1}^{t}$ é um isomorfismo se $f_{1}(i)$ e $f_{2}(i)$ são isomorfismos para todo $i=1, \ldots, t$.

Proposição 2.3.13. [11] Sejam $(\theta, \underline{Y})$ e $\left(\theta^{\prime}, \underline{Y}\right)$ sistemas estratificantes de tamanho t. Então existe um isomorfismo de sistemas estratificantes

$$
f:(\theta, \underline{Y}) \longrightarrow\left(\theta^{\prime}, \underline{Y}\right) .
$$

Proposição 2.3.14. [11] Sejam $(\theta, \underline{Y})$ e $\left(\theta, \underline{Y}^{\prime}\right)$ dois sistemas estratificantes de tamanho $t$. Então existem A-isomorfismos $f_{i}: Y(i) \longrightarrow Y^{\prime}(i)$, para cada $i=1, \ldots, t$, tais que

$$
f=\left\{1_{\theta(i)}, f_{1}(i)\right\}_{i=1}^{t}:(\theta, \underline{Y}) \longrightarrow\left(\theta, \underline{Y}^{\prime}\right)
$$

é um isomorfismo de sistemas estratificantes.

Definição 2.3.15. Sejam $\left(T_{1}, \ldots, T_{t}\right)$ uma sequência de módulos indecomponíveis não isomorfos e $T=T_{1} \oplus \cdots \oplus T_{t}$. Dizemos que a sequência $\left(T_{1}, \ldots, T_{t}\right)$ é uma sequência especial para $T$ se existir um sistema estratificante $\left(X_{1}, \ldots, X_{t}\right)$ tal que as seguintes duas condiçôes se verificam:

1. $\mathcal{F}(X) \cap \mathcal{Y}(X)=\operatorname{add}(T)$.

2. Existem sequências exatas da forma

$$
0 \longrightarrow X_{i} \longrightarrow T_{i} \rightarrow Z_{i} \longrightarrow 0
$$

com $Z_{i} \in \mathcal{F}\left(X_{1}, \ldots, X_{i-1}\right)$, para todo $i=1, \ldots, t$.

Notemos que, segundo a Proposição 2.3.13, dada uma sequência especial $\left(T_{1}, \ldots, T_{t}\right)$ de um módulo $T$ existe um único sistema estratificante associado a esta. Mais ainda, segundo a Proposição 2.3.14, dado um sistema estratificante existe uma única sequência especial $\left(T_{1}, \ldots, T_{t}\right)$ de um módulo $T$ associada a este. Como consequência temos que, se $A$ é uma álgebra heredi- 
tária existe uma bijeção entre as sequências especiais dos $A$-módulos inclinantes parciais com $t$ somandos diretos e os sistemas estratificantes sobre $A$ de tamanho $t$.

A seguir vamos enunciar dois resultados que aparecem em [6] (Lemas 1 e 2 respectivamente) e que usaremos com muita frequência ao longo deste trabalho.

Lema 2.3.16. [6] Sejam $K$ um corpo algebricamente fechado, A uma K-álgebra hereditária e $\left(X_{1}, \ldots, X_{a}, Z_{1}, \ldots, Z_{c}\right)$ uma sequência excepcional (não completa) sobre A. Então ela pode ser estendida a uma sequência excepcional completa na forma

$$
\left(X_{1}, \ldots, X_{a}, Y_{1}, \ldots, Y_{b}, Z_{1}, \ldots, Z_{c}\right)
$$

Lema 2.3.17. [6] Sejam $K$ um corpo algebricamente fechado, A uma K-álgebra hereditária e $E=\left(X_{1}, \ldots, X_{n}\right)$ e $F=\left(Y_{1}, \ldots, Y_{n}\right)$ sequências excepcionais completas sobre $A$ tais que $X_{i} \cong Y_{i}$ para todo $i \neq j$. Então $X_{j} \cong Y_{j}$.

A seguir usaremos estes dois lemas para construir todas os sistemas estratificantes sobre um tipo muito especial de álgebra.

Teorema 2.3.18. Seja $A$ a K-álgebra de caminhos dada pelo carcás

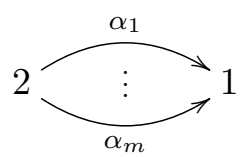

com $m \geq 2$. Então na seguinte lista estão todos os sistemas estratificantes completos sobre A.

1. $\left(P_{1}, P_{2}\right)$.

2. $\left(P_{2}, \tau^{-1} P_{2}\right)$.

3. $\left(\tau^{-i} P_{1}, \tau^{-i} P_{2}\right)$, com $i \geq 1$.

4. $\left(\tau^{-i} P_{2}, \tau^{-i-1} P_{1}\right)$, com $i \geq 1$.

5. $\left(I_{1}, I_{2}\right)$

6. $\left(I_{2}, P_{1}\right)$.

7. $\left(\tau I_{2}, I_{1}\right)$.

8. $\left(\tau^{i} I_{1}, \tau^{i} I_{2}\right)$, com $i \geq 1$.

9. $\left(\tau^{i+1} I_{2}, \tau^{i} I_{1}\right)$, com $i \geq 1$. 
Demonstração. Primeiro vamos observar que os módulos regulares indecomponíveis sobre a álgebra considerada têm auto-extensões, isto é se $M$ é um $A$-módulo regular e indecomponível então $\operatorname{Ext}_{A}^{1}(M, M) \neq 0$. De fato, para $m=2$ estes estão sobre um tubo de posto 1 do carcás de Auslander-Reiten e para $m>2$ podemos concluir que não tem auto-extensões como consequência do Corolário 2.16 Cap. XVIII em [18]. Assim qualquer sistema estratificante sobre esta álgebra não pode conter módulos regulares.

Para construir um sistema estratificante completo sobre $A$ vamos usar $A$-módulos pósprojetivos ou pré-injetivos indecomponíveis uma vez que estes não têm auto-extensões.

Vamos fazer a verificação de que os pares em 1 e 3 da lista são sistemas estratificantes completos. As outras verificações são análogas.

Para o par $\left(P_{1}, P_{2}\right)$ é só notar que $P_{1}$ é um projetivo simples e, portanto, $\operatorname{Hom}_{A}\left(P_{2}, P_{1}\right)=0$.

Para o par $\left(\tau^{-i} P_{1}, \tau^{-i} P_{2}\right)$, com $i \geq 1$, pelo Corolário 2.15 (c) em [2], temos que

$$
\operatorname{Hom}_{A}\left(\tau^{-i} P_{2}, \tau^{-i} P_{1}\right) \cong \operatorname{Hom}_{A}\left(P_{2}, P_{1}\right)
$$

e então $\operatorname{Hom}_{A}\left(\tau^{-i} P_{2}, \tau^{-i} P_{1}\right)=0$. Por outro lado, usando a fórmula de Auslander, segue que

$$
\begin{aligned}
\operatorname{Ext}_{A}^{1}\left(\tau^{-i} P_{2}, \tau^{-i} P_{1}\right) & =D \operatorname{Hom}_{A}\left(\tau^{-i} P_{1}, \tau^{-i+1} P_{2}\right) \\
& =D \operatorname{Hom}_{A}\left(\tau^{-1} P_{1}, P_{2}\right) \\
& =0
\end{aligned}
$$

Assim $\left(\tau^{-i} P_{1}, \tau^{-i} P_{2}\right)$, com $i \geq 1$, é um sistema estratificante completo sobre $A$.

Para finalizar devemos garantir que a lista está completa. Para isto, segundo o Lema 2.3.16, é suficiente com que para cada $A$-módulo pós-projetivo ou pré-injetivo $M$ existam sequências excepcionais da forma $\left(M, M^{\prime}\right)$ e $\left(M^{\prime \prime}, M\right)$. Isto de fato acontece.

Sejam $A$ uma álgebra hereditária e $X=\left(X_{1}, \ldots, X_{t}\right)$ um sistema estratificante sobre $A$. Denotamos por $C(X)$ a menor subcategoria plena de $\bmod A$ que contém $X$ e que é fechada por extensões.

Lema 2.3.19. Seja $X$ um sistema estratificante sobre a $K$-álgebra $A$ (não necessariamente hereditária), então $\mathcal{F}(X) \subseteq C(X)$.

Demonstração. Seja $M \in \mathcal{F}(X)$. Faremos a prova por indução no $X$-comprimento de $M$. Se $M \in X$, não temos nada a provar. Seja $M$ tal que $\ell_{X}(M)=m$, com $1 \leq m$ e suponhamos que a afirmação vale para todos os módulos em $\mathcal{F}(X)$ tais que seu $X$-comprimento é menor do que $m$. Consideremos a $X$-filtração para $M$ dada por

$$
0 \subseteq M_{1} \subseteq M_{2} \subseteq \ldots M_{m-1} \subseteq M_{m}=M
$$

Como $\ell_{X}\left(M_{m-1}\right)<m$ e $M_{m-1} \in \mathcal{F}(X)$, pela hipótese de indução $M_{m-1} \in C(X)$. Por outro 
lado, $M_{m} / M_{m-1} \cong X_{j}$ para algum $j \in\{1, \ldots, t\}$. Portanto, usando a sequência exata

$$
0 \longrightarrow M_{m-1} \longrightarrow M_{m} \longrightarrow M_{m} / M_{m-1} \longrightarrow 0
$$

concluímos que $M \in C(X)$, pois $C(X)$ é fechada por extensões.

Proposição 2.3.20. Sejam A uma álgebra (não necessariamente hereditária) e $X=\left(X_{1}, \ldots, X_{t}\right)$ um sistema estratificante tal que todos os seus elementos são A-módulos pós-projetivos. Então todos os módulos em $\mathcal{F}(X)$ são pós-projetivos. Em particular, se $T$ é tal que

$$
\mathcal{F}(X) \cap \mathcal{Y}(X)=\operatorname{add} T,
$$

então, $T$ é um módulo pós-projetivo.

Demonstração. Desde que $\mathcal{F}(X)$ é fechada por somas diretas é suficiente provar a afirmação para os $A$-módulos indecomponíveis nesta categoria. Suponhamos que $M \in \mathcal{F}(X)$ é um $A$ módulo indecomponível e que tem uma $X$-filtração da forma

$$
0 \subseteq M_{1} \subseteq M_{2} \subseteq \ldots M_{l-1} \subseteq M_{l}=M
$$

Então existe um epimorfismo $f: M_{l} \longrightarrow M_{l} / M_{l-1}$, tal que $M_{l} / M_{l-1} \cong X_{i}$, para algum $i \in$ $\{1, \ldots, t\}$. Como a categoria dos módulos pós-projetivos é fechada por predecessores concluímos que $M$ é pós-projetivo.

De maneira análoga provamos o seguinte resultado quando os elementos de $X$ são módulos pré-injetivos.

Proposição 2.3.21. Sejam A uma álgebra (não necessariamente hereditária) e $X=\left(X_{1}, \ldots, X_{t}\right)$ um sistema estratificante tal que todos os seus elementos são A-módulos pré-injetivos. Então todos os módulos em $\mathcal{F}(X)$ são pré-injetivos. Em particular, se $T$ é tal que

$$
\mathcal{F}(X) \cap \mathcal{Y}(X)=\operatorname{add} T,
$$

então T é um módulo pré-injetivo.

Definição 2.3.22. Seja $A$ uma $K$-álgebra. $\operatorname{Um} \operatorname{par}(\mathcal{T}, \mathcal{F})$ de subcategorias plenas de $\bmod A$ é chamado um par de torção se satisfaz as condiçôes 1, 2 e 3 abaixo:

1. $\operatorname{Hom}_{A}(M, N)=0$, para todo $M \in \mathcal{T}$ e para todo $N \in \mathcal{F}$.

2. $\left.\operatorname{Hom}_{A}(M,-)\right|_{\mathcal{F}}=0$ implica que $M \in \mathcal{T}$. 
3. $\left.\operatorname{Hom}_{A}(\ldots, N)\right|_{\mathcal{T}}=0$ implica que $M \in \mathcal{F}$.

A categoria $\mathcal{F}$ é chamada a classe livre de torção e a categoria $\mathcal{T}$ é chamada a classe de torção.

Seja $M$ é um $A$-módulo. A classe dos $A$-módulos $N$ tais que existe um inteiro $d \geq 0$ e um epimorfismo de $A$-módulos $M^{d} \longrightarrow N$ é denotada por Gen $M$. Dualmente, a classe Cogen $M$ é a classe dos $A$-módulos $N$ tais que existe um inteiro $d \geq 0$ e um monomorfismo de $A$-módulos $N \longrightarrow M^{d}$.

Proposição 2.3.23. Seja T um A-módulo inclinante parcial. Então:

1. (2.3 Cap. VI em [2])

(a) Gen T é uma classe de torção e a sua classe livre de torção correspondente é

$$
\mathcal{F}(T)=\left\{M \in \bmod A / \operatorname{Hom}_{A}(T, M)=0\right\} .
$$

(b) $\mathcal{T}(T)=\left\{M \in \bmod A / \operatorname{Ext}_{A}^{1}(T, M)=0\right\}$ é uma classe de torção e a sua classe livre de torção correspondente é Cogen $\tau T$.

2. (2.5 Cap. VI em [2]) As seguintes condiçôes são equivalentes:

(a) Té um A-módulo inclinante.

(b) Gen $T=\mathcal{T}(T)$.

(c) $\mathcal{F}(T)=$ Cogen $\tau T$.

(d) Seja $X$ um A-módulo. Então $X \in \operatorname{add} T$ se, e somente se, $X$ é relativamente projetivo em $\mathcal{T}(T)$.

(e) Para cada módulo $M \in \mathcal{T}(T)$ existe uma sequência exata

$$
0 \longrightarrow L \longrightarrow T_{0} \longrightarrow M \longrightarrow 0
$$

com $T_{0} \in \operatorname{add} T$ e $L \in \mathcal{T}(T)$.

Como consequência da anterior proposição, se $T$ é um módulo inclinante o par

$$
(\operatorname{Gen} T, \mathcal{F}(T))=(\mathcal{T}(T), \operatorname{Cogen} \tau T)
$$

é um par de torção. Tal par é chamado par de torção induzido por $T$ em $\bmod A$ e o denotamos por $(\mathcal{T}(T), \mathcal{F}(T))$.

Vamos estudar algumas relações deste par de torção com o sistema estratificante ao qual $T$ esta associado. 
Observação 2.3.24. É importante ressaltar que embora seja usada uma notação parecida para denotar a classe livre de torção induzida por um módulo inclinante $T$ e a categoria dos módulos filtrados por um sistema estratificante $X$ o contexto sempre permitirá entender a qual dos dois objetos estamos nos referindo.

Se $X$ é um sistema estratificante sobre uma álgebra hereditária e $\mathcal{F}(X) \cap \mathcal{Y}(X)=\operatorname{add} T$, então, pelo Lema 2.3.20, $X \nsubseteq \mathcal{F}(T)$. De fato, caso contrário como $\mathcal{F}(T)$ é fechada por extensões teríamos que $\mathcal{F}(X) \subseteq \mathcal{F}(T)$ e, em particular, que $T \in \mathcal{F}(T)$, o qual é impossível.

Proposição 2.3.25. Sejam A uma álgebra hereditária, $X=\left(X_{1}, \ldots, X_{n}\right)$ um sistema estratificante completo sobre $A, T$ um A-módulo inclinante básico e $T=\oplus_{i=1}^{n} T_{i}$ sua decomposição em somandos diretos indecomponíveis. Se $\left(T_{1}, \ldots, T_{n}\right)$ é uma sequência especial de $T$ associada a $X$, então as condições que seguem são equivalentes:

1. $X \subseteq \mathcal{T}(T)$.

2. $\mathcal{F}(X) \subseteq \mathcal{T}(T)$.

3. $\left.\operatorname{Ext}_{A}^{1}(T,-)\right|_{\mathcal{F}(X)}=0$.

4. $\mathcal{F}(X)=\operatorname{add} T$.

5. $X_{i}=T_{i}$, para $i=1,2, \ldots, n$.

6. $\mathcal{F}(X)=\operatorname{add}\left(X_{1} \oplus \ldots \oplus X_{n}\right)$.

Demonstração. $(1 \Rightarrow 2)$ Segue do Lema 2.3.19, pois $\mathcal{T}(T)$ é fechada por extensões.

$(2 \Leftrightarrow 3)$ Uma vez que $\mathcal{T}(T)=\left\{M \in \bmod A / \operatorname{Ext}_{A}^{1}(T, M)=0\right\}$ esta equivalência é clara.

$(2 \Rightarrow 4)$ Seja $M$ um $A$-módulo em $\mathcal{F}(X)$. Segundo a Proposição 2.3.23, para provar que $M \in \operatorname{add} T$ é suficiente mostrar que $\left.\operatorname{Ext}_{A}^{1}(M,-)\right|_{\mathcal{T}(T)}=0$. Se $N \in \mathcal{T}(T)$, pela Proposição 2.3.23, existe uma sequência exata da forma

$$
0 \longrightarrow L \longrightarrow T_{0} \longrightarrow N \longrightarrow 0
$$

com $T_{0} \in$ add $T$ e $L \in \mathcal{T}(T)$. Aplicamos o funtor $\operatorname{Hom}_{A}\left(M, \_\right.$) nesta sequência e obtemos

$$
\cdots \longrightarrow \operatorname{Ext}_{A}^{1}(M, L) \longrightarrow \operatorname{Ext}_{A}^{1}\left(M, T_{0}\right) \longrightarrow \operatorname{Ext}_{A}^{1}(M, N) \longrightarrow 0 .
$$

Como $\operatorname{Ext}_{A}^{1}\left(M, T_{0}\right)=0$, pois pela hipótese $\mathcal{F}(X) \cap \mathcal{Y}(X)=\operatorname{add} T$, então $\operatorname{Ext}_{A}^{1}(M, N)=0$, deste modo $\mathcal{F}(X) \subseteq$ add $T$. Por outro lado, como $T \in \mathcal{F}(X)$ e a categoria $\mathcal{F}(X)$ é fechada por somas diretas e por somandos diretos, então add $T \subseteq \mathcal{F}(X)$.

Para $(4 \Rightarrow 5)$ suponhamos que $\mathcal{F}(X)=\operatorname{add} T$. Como para cada $i \in\{1,2, \ldots, n\}$ o $A$-módulo $X_{i}$ é indecomponível e $X_{i} \in \mathcal{F}(X)$, então $\operatorname{add} T=\operatorname{add}\left(X_{1} \oplus \ldots \oplus X_{n}\right)$. 
Por outro lado, como $\left(T_{1}, \ldots, T_{n}\right)$ é uma sequência especial de $T$ associada ao sistema estratificante $X$, então add $T=\mathcal{Y}(X) \cap \mathcal{F}(X)$ e para $i \in\{1,2, \ldots, n\}$, existe uma sequência exata da forma

$$
0 \longrightarrow X_{i} \longrightarrow T_{i} \longrightarrow Z_{i} \longrightarrow 0
$$

onde $Z_{i} \in \mathcal{F}\left(X_{1}, \ldots, X_{i-1}\right)$. Desta maneira, temos as seguintes igualdades

$$
\operatorname{add}\left(X_{1} \oplus \ldots \oplus X_{n}\right)=\operatorname{add} T=\mathcal{Y}(X) \cap \mathcal{F}(X)=\mathcal{F}(X)
$$

Portanto, se $Z_{i} \neq 0$ a sequência exata acima cinde, pois $X_{i}$ e $Z_{i}$ estão em add $T$. Mas isto não é possível pois $T_{i}$ é um $A$-módulo indecomponível. Logo $Z_{i}=0$ e, portanto, $X_{i}=T_{i}$.

Claramente $(5 \Rightarrow 1)$.

$(5 \Rightarrow 6)$ Suponhamos que $X_{i}=T_{i}$, para $i=1, \ldots, n$. Como $\mathcal{F}(X)$ é fechada por somandos diretos então $\operatorname{add}\left(X_{1} \oplus \ldots \oplus X_{n}\right)=\operatorname{add}\left(T_{1} \oplus \ldots \oplus T_{n}\right) \subseteq \mathcal{F}(X)$.

Seja $M \in \mathcal{F}(X)$. Vamos demonstrar que $M \in \operatorname{add}\left(X_{1} \oplus \ldots \oplus X_{n}\right)$ por indução no $X$ comprimento de $M$. Se $M \in X$ não temos nada a provar. Seja $M$ tal que $\ell_{X}(M)=m$, com $m \geq 1$, e suponhamos que a afirmação vale para os módulos em $\mathcal{F}(X)$ tais que o seu $X$ comprimento é menor do que $m$. Consideramos a $X$-filtração para $M$ dada por

$$
0 \subseteq M_{1} \subseteq M_{2} \subseteq \ldots M_{m-1} \subseteq M_{m}=M
$$

para construir a sequência exata

$$
0 \longrightarrow M_{m-1} \longrightarrow M \longrightarrow M / M_{m-1} \longrightarrow 0
$$

Como $\ell_{X}\left(M_{m-1}\right)<m$, pela hipótese de indução, $M_{l-1} \in \operatorname{add}\left(T_{1} \oplus \ldots \oplus T_{n}\right)$. Por outro lado, $M / M_{m-1} \cong T_{i}$, para algum $i \in\{1, \ldots, t\}$. Portanto a sequência $(*)$ cinde, o que implica que $M \in \operatorname{add}\left(X_{1} \oplus \ldots \oplus X_{n}\right)$. Concluímos que

$$
\operatorname{add}\left(X_{1} \oplus \ldots \oplus X_{n}\right)=\operatorname{add} T=\mathcal{F}(X)
$$

$(6 \Rightarrow 5)$ Suponhamos que $\mathcal{F}(X)=\operatorname{add}\left(X_{1} \oplus \ldots, \oplus X_{n}\right)$. Como add $T \subseteq \mathcal{F}(X)$, então add $T \subseteq$ $\operatorname{add}\left(X_{1} \oplus \ldots \oplus X_{n}\right)$. Desde que $T$ tem $n$ somandos diretos não isomorfos $\mathcal{F}(X)=$ add $T$. Uma vez que a implicação $(4 \Rightarrow 5)$ é válida concluímos que $X_{i}=T_{i}$, para $i=1, \ldots, n$.

Exemplo 2.3.26. Seja $A=K Q$, onde $Q$ é o carcás

$Q:$

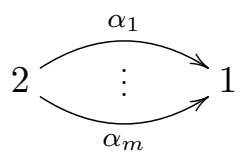


com $m \geq 2$. Usaremos a lista de sistemas estratificantes obtida na Proposição 2.3 .18 para fornecer exemplos de sistemas estratificantes, satisfazendo as condições da proposição anterior, mas cujos elementos não são todos módulos injetivos ou projetivos. Notemos que em tal lista todos os sistemas estratificantes, exceto $\left(I_{2}, P_{1}\right)$, estão formados por módulos ou somente pós-projetivos ou somente pré-injetivos. Mais ainda, se $X=\left(X_{1}, X_{2}\right)$ é um sistema estratificante completo sobre a álgebra $A$ e $X \neq\left(I_{2}, P_{1}\right)$, então $\mathcal{F}(X)=\operatorname{add}\left(X_{1} \oplus X_{2}\right)$.

Vejamos primeiro que para o sistema estratificante $\left(I_{2}, P_{1}\right)$ não vale tal igualdade. Para tal calculemos a matriz de Cartan de $A$ e a sua inversa. Temos, então, que

$$
C_{A}=\left[\begin{array}{cc}
1 & m \\
0 & 1
\end{array}\right] \quad \text { e } \quad C_{A}^{-1}=\left[\begin{array}{cc}
1 & -m \\
0 & 1
\end{array}\right]
$$

Portanto, a matriz de Coxeter de $A$, que esta definida por $\Phi_{A}=-C_{A}^{t} C_{A}^{-1}$, é igual a

$$
\Phi_{A}=\left[\begin{array}{cc}
-1 & m \\
-m & m^{2}-1
\end{array}\right]
$$

Pela Proposição 1.6.7, temos que

$$
\underline{\operatorname{dim}} \tau I_{2}=\Phi_{A}\left(\underline{\operatorname{dim}} I_{2}\right)=\left[\begin{array}{cc}
-1 & m \\
-m & m^{2}-1
\end{array}\right]\left[\begin{array}{c}
1 \\
m
\end{array}\right]=\left[\begin{array}{c}
m^{2}-1 \\
m\left(m^{2}-2\right)
\end{array}\right]
$$

e, desde que $m^{2}-1 \neq 0$ para $m \neq 1$, pelo Corolário 1.5 .5 , temos que

$$
\left[\tau I_{2}, S_{1}\right]=\operatorname{dim}_{K} \operatorname{Hom}_{A}\left(P_{1}, \tau I_{2}\right)=m^{2}-1 \neq 0 .
$$

Por outro lado, usando a fórmula de Auslander, resulta que

$$
\operatorname{Ext}_{A}^{1}\left(I_{2}, P_{1}\right) \cong D \operatorname{Hom}_{A}\left(P_{1}, \tau I_{2}\right) \neq 0
$$

Isto significa que $P_{1}$ não é um módulo relativamente injetivo na categoria $\mathcal{F}(X)$, e portanto, $\mathcal{F}\left(I_{2}, P_{1}\right) \neq \operatorname{add}\left(I_{2} \oplus P_{1}\right)$.

Para o sistema estratificante $\left(\tau^{i+1} I_{2}, \tau^{i} I_{1}\right)$, temos que $\tau^{i} I_{1}$ é relativamente injetivo em $\mathcal{F}\left(\tau^{i} I_{1}, \tau^{i+1} I_{2}\right)$. De fato, pela fórmula de Auslander, temos que

$$
\operatorname{Ext}_{A}^{1}\left(\tau^{i+1} I_{2}, \tau^{i} I_{1}\right) \cong D \operatorname{Hom}_{A}\left(\tau^{i} I_{1}, \tau^{i+2} I_{2}\right) \cong D \operatorname{Hom}_{A}\left(I_{1}, \tau^{2} I_{2}\right)=0
$$

Portanto $\mathcal{F}\left(\tau^{i+1} I_{2}, \tau^{i} I_{1}\right)=\operatorname{add}\left(\tau^{i+1} I_{2} \oplus \tau^{i} I_{1}\right)$. Para os demais sistemas estratificantes da lista vale a mesma afirmação e a verificação é análoga. 


\section{Capítulo 3}

\section{Sistemas estratificantes de módulos regulares sobre álgebras hereditárias euclidianas}

Se $A$ é uma álgebra hereditária existe uma relação estreita entre os sistemas estratificantes sobre $A$ e os $A$-módulos inclinantes. Uma questão de interesse é analisar a existência de um sistema estratificante completo formado somente de módulos regulares.

Se $A$ é uma álgebra de Kronecker ou uma Kronecker generalizada todos os módulos regulares têm auto-extensões e, portanto, um sistema estratificante sobre $A$ não tem elementos regulares.

Se $A=K Q$ e o grafo subjacente $\bar{Q}$ de $Q$ é um dos diagramas de Dynkin, então $\bmod A$ não tem módulos regulares. Assim, neste caso, também não é possível construir um sistema estratificante formado por módulos regulares.

Neste capítulo demonstraremos que, se $\bar{Q}$ é um diagrama euclidiano, não é possível construir um sistema estratificante com tais características e encontramos uma limitante para o tamanho de um sistema estratificante formado de módulos regulares. Mais ainda, mostramos que não é possível construir tal sistema estratificante em um tipo mais geral de álgebras: as álgebras disfarçadas de tipo euclideano.

\subsection{Sistemas estratificantes de módulos regulares sobre álgebras hereditárias euclidianas}

Demostraremos que em um tubo $\mathcal{T}_{\lambda}$ estândar, hereditário e estável de posto $r \geq 1 \mathrm{em}$ $\Gamma(\bmod A)$ pode-se construir um módulo inclinante parcial e básico $T$ com no máximo $r-1$ somandos em add $\mathcal{T}_{\lambda}$. Como uma consequência concluimos que, sobre uma álgebra hereditária euclidiana $A$, um sistema estratificante formado de módulos regulares tem no máximo tamanho $n-2$, onde $n$ é o posto de $K_{0}(A)$.

Começaremos enunciando alguns lemas técnicos. 
Definição 3.1.1. Seja $(\mathcal{T}, \tau)$ um tubo estável. Então

1. O conjunto dos pontos em $\mathcal{T}$ que têm exatamente um predecessor imediato (ou equivalentemente exatamente um sucessor imediato) é chamado boca de $\mathcal{T}$.

2. Dado um ponto $x$ na boca de $\mathcal{T}$, o raio começando em $x$ é o único caminho seccional infinito

$$
x=x[1] \rightarrow x[2] \rightarrow x[3] \rightarrow \cdots \rightarrow x[m] \rightarrow \cdots
$$

Lema 3.1.2. (1.4 Cap. X em [17]) Seja $A$ uma álgebra e $\mathcal{T}_{\lambda}$ um tubo estável de posto $r_{\lambda} \geq 1$ em $\Gamma(\bmod A)$. Se $\left(X_{1}, \ldots, X_{r_{\lambda}}\right)$ é um $\tau$-ciclo de A-módulos na boca do tubo $\mathcal{T}_{\lambda}$. Então

1. Para cada $i \in\left\{1, \ldots, r_{\lambda}\right\}$ existe um único raio

$$
X_{i}=X_{i}[1] \rightarrow X_{i}[2] \rightarrow X_{i}[3] \rightarrow \cdots \rightarrow X_{i}[m] \rightarrow \cdots
$$

2. Cada A-módulo indecomponivel $M$ em $\mathcal{T}_{\lambda}$ é da forma $M \cong X_{i}[m]$, para algum $i \in$ $\left\{1, \ldots, r_{\lambda}\right\}$ e $m \geq 1$.

Para um módulo indecomponível $M \cong X_{i}[j]$ num tubo estável $\mathcal{T}_{\lambda}$ o cone determinado por $M$, que denotamos por $\mathcal{C}(M)$, consiste dos $A$-módulos $X_{s}[u]$, com $s \in\{i, i+1, \ldots, i+j-1\}$ e $u \leq i+j-s$. Além disso, o inteiro $j$ é denotado por $\ell_{\lambda}(M)$. Graficamente o cone de $M$ tem a seguinte forma

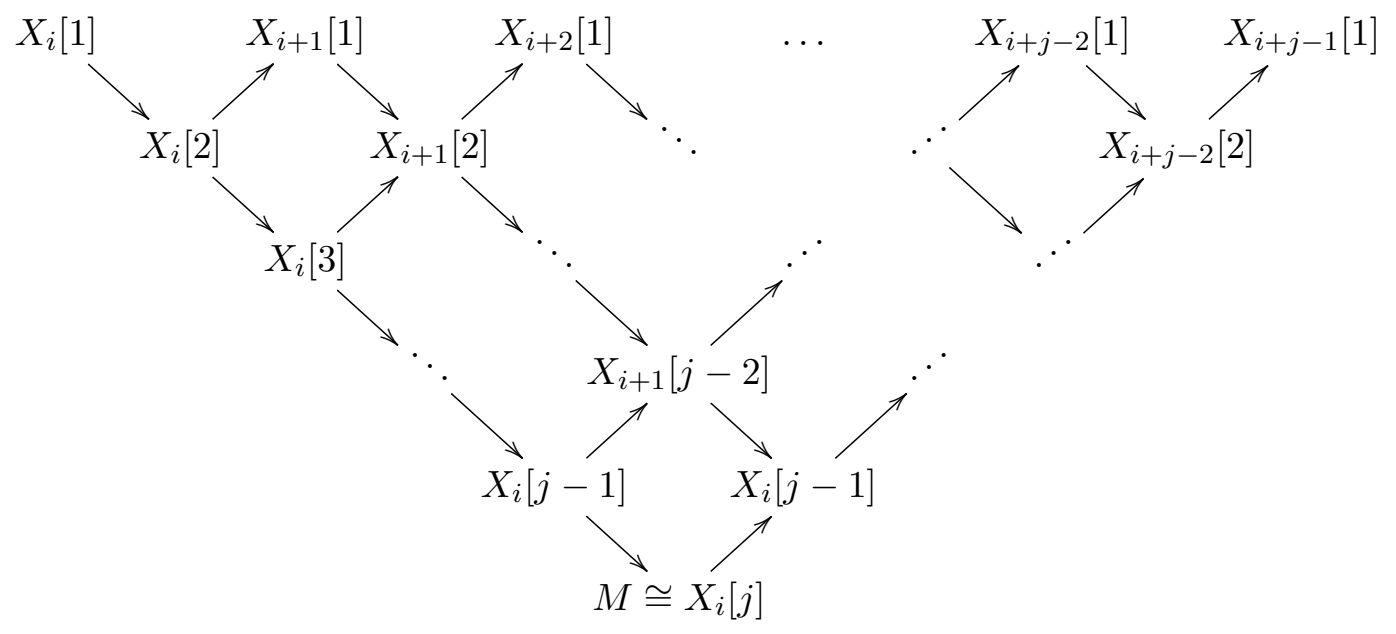

Lema 3.1.3 (1.7 Cap. XVII em [18]). Sejam A uma álgebra $e \mathcal{T}_{\lambda}$ um tubo estável, estândar e hereditário de $\Gamma(\bmod A)$. Para cada par de A-módulos indecomponíveis $M$ e $N$ em add $\mathcal{T}_{\lambda}$ tais que $\operatorname{Ext}_{A}^{1}(M \oplus N, M \oplus N)=0$, uma das seguintes condições é satisfeita: 
1. $\mathcal{C}(M) \subseteq \mathcal{C}(N)$,

2. $\mathcal{C}(N) \subseteq \mathcal{C}(M)$ ou

3. $\mathcal{C}(M) \cap \mathcal{C}(N)=\emptyset, \mathcal{C}(M) \cap \tau \mathcal{C}(N)=\emptyset$ e $\tau \mathcal{C}(M) \cap \mathcal{C}(N)=\emptyset$.

Corolário 3.1.4. Sejam A uma álgebra e $\mathcal{T}_{\lambda}$ um tubo estável, estândar e hereditário de $\Gamma(\bmod A)$ de posto $r_{\lambda} \geq 1$. Se $N_{1}, \ldots, N_{t}$ são A-módulos indecomponíveis e dois a dois não isomorfos em $\operatorname{add} \mathcal{T}_{\lambda}$, tais que $\operatorname{Ext}_{A}^{1}\left(N_{1} \oplus \ldots \oplus N_{t}, N_{1} \oplus \ldots \oplus N_{t}\right)=0$ e que $\mathcal{C}\left(N_{i}\right) \cap \mathcal{C}\left(N_{j}\right)=\emptyset$, sempre que $i \neq j$, então

$$
\ell_{\lambda}\left(N_{1}\right)+\cdots+\ell_{\lambda}\left(N_{t}\right) \leq r_{\lambda}-t
$$

Demonstração. Seja $\left(X_{1}, \ldots, X_{r_{\lambda}}\right)$ um $\tau$-ciclo na boca do tubo $\mathcal{T}_{\lambda}$. Para $i=1, \ldots, t$ vamos denotar por $\mathcal{N}_{i}$ e $\tau \mathcal{N}_{i}$ os conjuntos

$$
\mathcal{N}_{i}=\mathcal{C}\left(N_{i}\right) \cap\left\{X_{1}, \ldots, X_{r_{\lambda}}\right\} \quad \text { e } \quad \tau \mathcal{N}_{i}=\tau \mathcal{C}\left(N_{i}\right) \cap\left\{X_{1}, \ldots, X_{r_{\lambda}}\right\}
$$

Segundo o Lema 3.1.2 existem inteiros $l_{i}$ e $t_{i}$ tais que $N_{i} \cong X_{l_{i}}\left[t_{i}\right]$, para cada $i \in\{1, \ldots, t\}$. Então $\mathcal{N}_{i}=\left\{X_{l_{i}}[1], \ldots, X_{l_{i}+t_{i}-1}[1]\right\}$ e $\tau \mathcal{N}_{i}=\left\{X_{l_{i}-1}[1], \ldots, X_{l_{i}+t_{i}-2}[1]\right\}$. Portanto

$$
\left|\mathcal{N}_{i} \cup \tau \mathcal{N}_{i}\right|=t_{i}+1=\ell_{\lambda}\left(N_{i}\right)+1
$$

Como $\operatorname{Ext}_{A}^{1}\left(N_{i} \oplus N_{j}, N_{i} \oplus N_{j}\right)=0$ e $\mathcal{C}\left(N_{i}\right) \cap \mathcal{C}\left(N_{j}\right)=\emptyset$, sempre que $i \neq j$, pelo Lema 3.1.3, temos que $\mathcal{C}\left(N_{i}\right) \cap \tau \mathcal{C}\left(N_{j}\right)=\emptyset$ e que $\tau \mathcal{C}\left(N_{i}\right) \cap \mathcal{C}\left(N_{j}\right)=\emptyset$. Portanto

$$
\left|\bigcup_{i=1}^{t}\left(\mathcal{N}_{i} \cup \tau \mathcal{N}_{i}\right)\right|=\left(\sum_{i=1}^{t} \ell_{\lambda}\left(N_{i}\right)\right)+t .
$$

Mas por outro lado,

$$
\bigcup_{i=1}^{t}\left(\mathcal{N}_{i} \cup \tau \mathcal{N}_{i}\right) \subseteq\left\{X_{1}, \ldots, X_{r_{\lambda}}\right\}
$$

Logo

$$
\sum_{i=1}^{t} \ell_{\lambda}\left(N_{i}\right) \leq r_{\lambda}-t
$$

Lema 3.1.5. (1.8 Cap. XVII em [18]) Sejam A uma álgebra, $\mathcal{T}_{\lambda}$ um tubo estândar, hereditário e estável de $\Gamma(\bmod A)$ tal que $r_{\lambda} \geq 1$ e $M$ um A-módulo indecomponível em add $\mathcal{T}_{\lambda}$ tal que $\operatorname{Ext}_{A}^{1}(M, M)=0$. Se $\left\{X_{1}, \ldots, X_{p}\right\}$ é um conjunto não vazio de A-módulos indecomponíveis $e$ 
dois a dois não isomorfos no cone $\mathcal{C}(M)$ determinado por $M$ tal que

$$
\operatorname{Ext}_{A}^{1}\left(X_{1} \oplus \ldots \oplus X_{p}, X_{1} \oplus \ldots \oplus X_{p}\right)=0 .
$$

Então $p \leq \ell_{\lambda}(M)$.

Proposição 3.1.6. Sejam $A$ uma álgebra, $\mathcal{T}_{\lambda}$ um tubo estândar, hereditário e estável de $\Gamma(\bmod A)$ tal que $r_{\lambda} \geq 1$. Se Té um A-módulo inclinante básico, então $T$ tem no máximo $r_{\lambda}-1$ somandos diretos em add $\mathcal{T}_{\lambda}$.

Demonstração. Seja $T$ um $A$-módulo inclinante. Definimos as classes $\mathcal{X}$ e $\mathcal{X}_{0}$ da seguinte forma

$$
\begin{aligned}
\mathcal{X} & =\left\{X \in \operatorname{ind} A / X \in \operatorname{add} \mathcal{T}_{\lambda} \cap \operatorname{add} T\right\} \text { e } \\
\mathcal{X}_{0} & =\{X \in \mathcal{X} / \forall Y \in \mathcal{X}, \mathcal{C}(Y) \subseteq \mathcal{C}(X) \text { ou } \mathcal{C}(X) \cap \mathcal{C}(Y)=\emptyset\}
\end{aligned}
$$

Como $T$ é um módulo inclinante básico, então $T$ tem um número finito de somandos diretos indecomponíveis não isomorfos e, portanto, a classe $\mathcal{X}$ tem finitos elementos.

Notemos que se $X_{1}, X_{2} \in \mathcal{X}_{0}$ e $X_{1} \neq X_{2}$, então $\mathcal{C}\left(X_{1}\right) \cap \mathcal{C}\left(X_{2}\right)=\emptyset$. De fato, em caso contrário teriamos que $\mathcal{C}\left(X_{1}\right)=\mathcal{C}\left(X_{2}\right)$ e portanto que $X_{1}=X_{2}$.

Assim, se o número de elementos de $\mathcal{X}_{0}$ é $l$, segundo o Corolário 3.1.4, temos que

$$
\sum_{X \in \mathcal{X}_{0}} \ell_{\lambda}(X) \leq r_{\lambda}-l
$$

Por outro lado, se $X \in \mathcal{X}_{0}$ então, pelo Lema 3.1.5, o número de elementos em $\mathcal{C}(X) \cap \mathcal{X}$ é no máximo $\ell_{\lambda}(X)$. Portanto

$$
|\mathcal{X}| \leq \sum_{X \in \mathcal{X}_{0}}|\mathcal{C}(X) \cap \mathcal{X}| \leq \sum_{X \in \mathcal{X}_{0}} \ell_{\lambda}(X) \leq r_{\lambda}-l
$$

Concluímos que se $\mathcal{X} \neq \emptyset$, então $|\mathcal{X}| \leq r_{\lambda}-1$.

Proposição 3.1.7. Sejam $K$ um corpo algebricamente fechado e A uma K-álgebra de dimensão finita. Seja $\mathcal{T}_{\lambda}$ um tubo estável, estândar e hereditário em $\Gamma(\bmod A)$ de posto $r \geq 1$. Então existe um sistema estratificante de tamanho $r-1$ contido em $\mathcal{T}_{\lambda}$. Mais ainda, se $X=\left(X_{1}, \ldots, X_{t}\right)$ é um sistema estratificante tal que $X_{i} \in \mathcal{T}_{\lambda}$, para todo $i=1, \ldots, t$, então $t \leq r-1$.

Demonstração. Seja $\left(X_{1}, \ldots, X_{r}\right)$ um $\tau$-ciclo na boca do tubo $\mathcal{T}_{\lambda}$. Isto é, os módulos $X_{i}$ estão ordenados de tal forma que

$$
\tau X_{2} \cong X_{1}, \ldots, \tau X_{r} \cong X_{r-1} \text { e } \tau X_{1} \cong X_{r}
$$

Vejamos que $X=\left(X_{r-1}, \ldots, X_{1}\right)$ é um sistema estratificante. Como $\mathcal{T}_{\lambda}$ é uma componente estândar então os $A$-módulos $X_{1}, \ldots, X_{r}$ são tijolos dois a dois ortogonais. Por outro lado, para 
$1<i \leq j \leq r-1$, pela fórmula de Auslander, temos que

$$
\operatorname{Ext}_{A}^{1}\left(X_{i}, X_{j}\right) \cong D \operatorname{Hom}_{A}\left(X_{j}, \tau X_{i}\right) \cong D \operatorname{Hom}_{A}\left(X_{j}, X_{i-1}\right)
$$

Como $j \neq i-1, \operatorname{Hom}_{A}\left(X_{j}, X_{i-1}\right)=0 . \operatorname{Assim} \operatorname{Ext}_{A}^{1}\left(X_{i}, X_{j}\right)=0$.

Se $i=1$ e $1 \leq j \leq r-1$, usando de novo a fórmula de Auslander, temos o isomorfismo

$$
\operatorname{Ext}_{A}^{1}\left(X_{1}, X_{j}\right) \cong D \operatorname{Hom}_{A}\left(X_{j}, \tau X_{1}\right) \cong D \operatorname{Hom}_{A}\left(X_{j}, X_{r}\right)
$$

Como $j \neq r$, temos que $\operatorname{Hom}_{A}\left(X_{j}, X_{r}\right)=0$ e portanto $\operatorname{Ext}_{A}^{1}\left(X_{1}, X_{j}\right)=0$.

Assim $X=\left(X_{r-1}, \ldots, X_{1}\right)$ é um sistema estratificante de tamanho $r-1$ com elementos em $\operatorname{add} \mathcal{T}_{\lambda}$.

Suponhamos que $Y=\left(Y_{1}, \ldots, Y_{t}\right)$ é um sistema estratificante tal que cada $Y_{i} \in \mathcal{T}_{\lambda}$. Pelo Teorema 1.7.9, temos que add $\mathcal{T}_{\lambda}=\mathcal{E} \mathcal{X} \mathcal{T}_{A}\left(E_{1}, \ldots, E_{r}\right)$ e, portanto, que add $\mathcal{T}_{\lambda}$ é uma subcategoria abeliana e fechada por extensões de $\bmod A$. Assim, usando o Lema 2.3.19, temos a seguinte cadeia de inclusões

$$
\mathcal{F}(Y) \subseteq C\left(Y_{1}, \ldots, Y_{t}\right) \subseteq \operatorname{add} \mathcal{T}_{\lambda}
$$

Por outro lado, do Lema 2.3.8, temos que $\mathcal{F}(Y) \cap \mathcal{Y}(Y)=$ add $T$, onde $T$ é um módulo inclinante parcial básico.

Mas, pela Proposição 3.1.6, o número de somandos diretos não isomorfos de $T$ é no máximo $r$ - 1. Dado que, pelo Teorema 2.1.6, o número de somandos diretos indecomponíveis de $T$ é igual ao tamanho de $X$ temos que $t \leq r-1$.

Com o intuito de dar uma aplicação da proposição precedente vamos definir uma classe de álgebras conhecidas como álgebras disfarçadas.

Definição 3.1.8. Sejam $Q$ um carcás finito, conexo e acíclico tal que $\bar{Q}$ não é um diagrama de Dynkin e $A=K Q$. Uma álgebra $B$ é chamada disfarçada tipo $Q$ se existir um A-módulo inclinante e pós-projetivo $T$ tal que $B=\operatorname{End}_{A}(T)$.

Notemos que, $\operatorname{como} \operatorname{End}_{A}(A) \cong A_{A}$, se $A=K Q$ é $Q$ e um carcás euclidiano então $A$ é uma álgebra disfarçada de tipo $Q$.

Teorema 3.1.9. (3.4 Cap. XI em [177]) Sejam $Q$ um carcás euclidiano, $A=K Q$ e $T_{A}$ um módulo inclinante pós-projetivo e básico. Sejam $B=\operatorname{End}_{A}\left(T_{A}\right)$ uma álgebra disfarçada de tipo $Q$ e add $\mathcal{R}(B)$ a subcategoria plena de $\bmod B$ cujos objetos são todos os $B$-módulos regulares. Então:

1. A categoria $\operatorname{add} \mathcal{R}(B)$ é abeliana e fechada por extensões.

2. As componentes de $\mathcal{R}(B)$ formam uma família $\mathcal{T}^{B}=\left\{\mathcal{T}_{\lambda}^{B}\right\}_{\lambda \in \Lambda}$ de tubos estáveis, estândares e dois a dois ortogonais. Mais ainda, se $r_{\lambda}^{B}$ denota o posto de $\mathcal{T}_{\lambda}^{B}$ e $n=\left|Q_{0}\right|$, 
então

$$
\sum_{\lambda \in \Lambda}\left(r_{\lambda}^{A}-1\right) \leq n-2
$$

3. Em $\mathcal{T}^{B}$ existem como máximo $n-2$ tubos não homogêneos. Isto é, todos exceto um número finito de tubos em $\mathcal{T}^{B}$ são homogêneos.

4. Para cada $\lambda \in \Lambda$, o tubo $\mathcal{T}_{\lambda}$ está composto por objetos indecomponiveis em uma subcategoria abeliana de $\bmod B$ da forma

$$
\mathcal{E}=\mathcal{E}_{A}=\mathcal{E} \mathcal{X} \mathcal{T}_{A}\left(E_{1}, \ldots, E_{r}\right)
$$

onde $E_{1}, \ldots, E_{r}$ são tijolos dois a dois ortogonais em $\bmod B$ tais que existe um isomorfismo $\tau E_{i+1} \cong E_{i}$, para todo $i \in\left\{1, \ldots, r_{\lambda}\right\}$, e $E_{1}=E_{r_{\lambda}}$.

Lema 3.1.10. (3.3 Cap. XI de [17]) Sejam Q um carcás acíclico cujo grafo subjacente é euclidiano, $A=K Q$ e $B$ uma álgebra disfarçada de tipo euclidiano $Q$. Então gd $B \leq 2$ e todos os $B$-módulos indecomponíveis $Z$, exceto um conjunto finito que consiste de módulos pós-projetivos ou pré-injetivos, são tais que $\operatorname{pd} Z \leq 1$ e id $Z \leq 1$.

Como consequência do lema acima temos que tubos em $\mathcal{T}^{B}$ são hereditários. Com isto temos o seguinte teorema.

Teorema 3.1.11. Sejam A uma álgebra disfarçada de tipo euclidiano e $n$ o posto de $K_{0}(A)$. Então existe um sistema estratificante cujos elementos são todos A-módulos regulares de tamanho $n-2$. Além disso, se $X$ é um sistema estratificante cujos elementos são A-módulos regulares então o tamanho de $X$ é no máximo $n-2$.

Demonstração. O resultado decorre diretamente da Proposição 3.1.7, do Teorema 3.1.9 e do Lema 3.1.10.

Como um caso particular temos o seguinte resultado.

Corolário 3.1.12. Sejam A uma álgebra hereditária euclidiana e $n$ o posto de $K_{0}(A)$. Então existe um sistema estratificante cujos elementos são todos A-módulos regulares de tamanho $n-2$. Além disso, se $X$ é um sistema estratificante cujos elementos são A-módulos regulares então o tamanho de $X$ é no máximo $n-2$. Isto é, não existe um sistema estratificante completo sobre A cujos elementos sejam todos regulares. 


\subsection{Sistemas estratificantes sobre álgebras de tipo $\Delta\left(\widetilde{\mathbb{A}}_{p, q}\right)$}

Segundo o Teorema 3.1.11, se $A=K Q$ é uma álgebra hereditária de tipo euclideano então é possível construir um sistema estratificante formado só de módulos regulares de tamanho no máximo $n-2$, onde $n=\left|Q_{0}\right|$. No caso particular da álgebra $\Delta\left(\widetilde{\mathbb{A}}_{p, q}\right)$ é possível construir um sistema estratificante formado por módulos regulares de tamanho no máximo $p+q-2$.

Nosso trabalho agora consiste em construir um sistema estratificante "regular" de tamanho maximal sobre $\Delta\left(\widetilde{\mathbb{A}}_{p, q}\right)$, para depois completa-lo de todas as formas possíveis.

Para isto primeiro apresentaremos uma lista dos módulos simples regulares em $\Delta\left(\widetilde{\mathbb{A}}_{p, q}\right)$ e um teorema que descreve a estrutura tubular da parte regular de tal álgebra. Usaremos a notação do Cap. XII em [17].

Construir o sistema estratificante "regular" de tamanho maximal é simples. Basta ordenar de forma conveniente os elementos nas bocas dos tubos não homogêneos. Completa-los é mais trabalhoso, pois é preciso encontrar módulos satisfazendo muitas propriedades.

\section{Lista: Módulos simples regulares de $\Delta\left(\widetilde{\mathbb{A}}_{p, q}\right)$}

Sejam $p$ e $q$ inteiros e suponhamos que $1 \leq p \leq q$. Seja $\Delta=\Delta\left(\widetilde{\mathbb{A}}_{p, q}\right)$ o carcás euclidiano orientado de forma canônica como segue

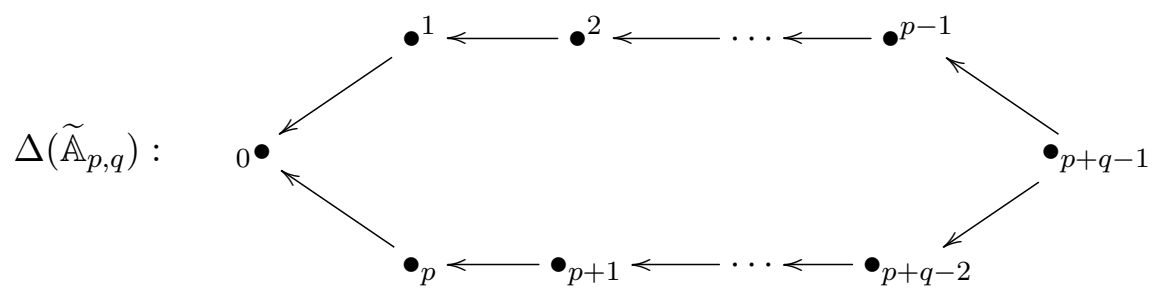

$\underline{\text { (a) Módulos simples regulares no tubo } \mathcal{T}_{\infty}^{\widetilde{\mathbb{A}}_{p, q}}}$

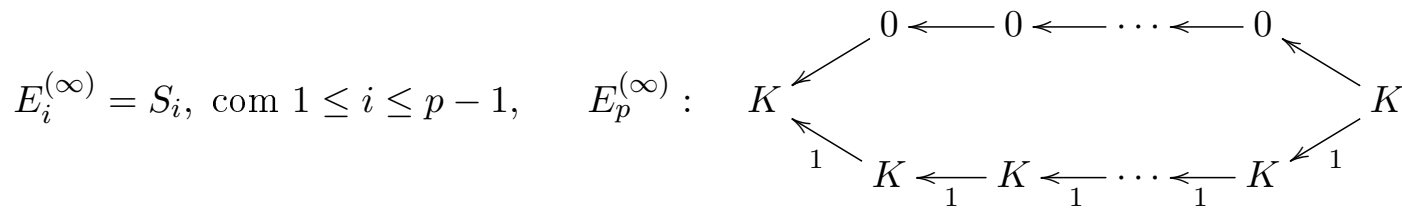

com a propriedade

$$
\tau E_{i+1}^{(\infty)}=E_{i}^{(\infty)}, \text { para } 1 \leq i \leq p-1, \quad \text { e } \quad \tau E_{1}^{(\infty)}=E_{p}^{(\infty)}
$$

(b) Módulos simples regulares no tubo $\mathcal{T}_{0}^{\widetilde{\mathbb{A}}_{p, q}}$

$E_{j}^{(0)}=S_{p+j-1}$, para $1 \leq j \leq q-1 \quad E_{q}^{(0)}:$

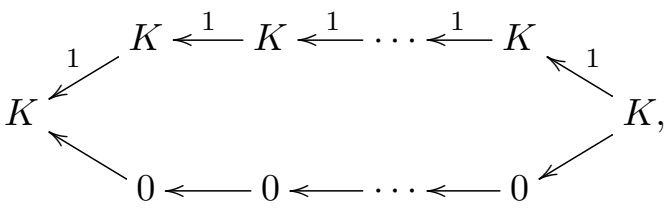


com a propriedade:

$$
\tau E_{j+1}^{0}=E_{j}^{0}, \text { para } 1 \leq j \leq q-1, \quad \text { e } \quad \tau E_{1}^{0}=E_{q}^{0} .
$$

(c) Módulos simples regulares no tubo $\mathcal{T}_{\lambda}^{\widetilde{\mathbb{A}}_{p, q}}$, com $\lambda \in K \backslash\{0\}$

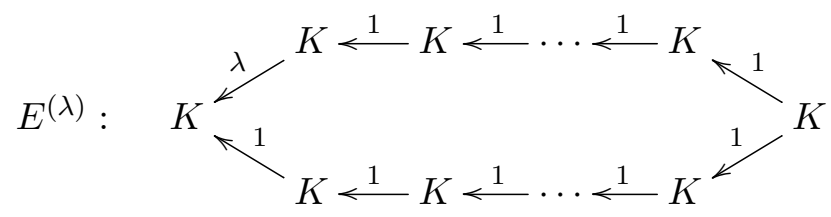

Vamos enunciar agora um teorema que descreve a estrutura tubular de $\Delta\left(\widetilde{\mathbb{A}}_{p, q}\right)$ e que usaremos para a construção de um sistema estratificante "regular"de tamanho máximo.

Teorema 3.2.1. (2.5 Cap. XIII em [177]) Assumamos que $A=K \Delta$, onde $\Delta$ é o carcás orientado em forma canônica $\Delta\left(\widetilde{\mathbb{A}}_{p, q}\right)$, com $q \geq p \geq 1$, como na lista acima.

1. Cada A-módulo simples regular é isomorfo a um dos módulos na lista acima.

2. As componentes regulares de $\mathcal{R}(A)$ em $\Gamma(\bmod A)$ são descritas na seguinte lista:

(a) O tubo $\mathcal{T}_{\infty}^{\widetilde{\mathbb{A}}_{p, q}}$ de posto $p$ contendo os A-módulos $E_{1}^{(\infty)}, \ldots, E_{p}^{(\infty)}$.

(b) $O$ tubo $\mathcal{T}_{0}^{\widetilde{\mathbb{A}}_{p, q}}$ de posto $q$ contendo os $A$-módulos $E_{1}^{(0)}, \ldots, E_{q}^{(0)}$.

(c) $O$ tubo $\mathcal{T}_{\lambda}^{\widetilde{\mathbb{A}}_{p, q}}$ de posto 1 contendo o A-módulo $E^{(\lambda)}$, com $\lambda \in K \backslash\{0\}$.

Onde $E_{j}^{(\infty)}, E_{i}^{(0)}$ e $E^{(\lambda)}$ são os A-módulos simples regulares na lista acima.

3. $O$ carcás de Auslander-Reiten $\Gamma(\bmod A)$ de $A$ consiste de uma componente pós-projetiva $\mathcal{P}(A)$, uma componente pré-injetiva $\mathcal{Q}(A)$ e a família

$$
\mathcal{T}^{Q}=\left\{\mathcal{T}_{\lambda}^{Q}\right\}_{\lambda \in \mathbb{P}_{1}(K)}
$$

de tubos estáveis separando $\mathcal{P}(A)$ de $\mathcal{Q}(A)$.

A seguir usaremos os módulos simples regulares sobre os tubos de posto $p$ e $q$ para construir um sistema estratificante de tamanho $p+q-2$ sobre $\Delta\left(\widetilde{\mathbb{A}}_{p, q}\right)$.

Por conveniência, denotaremos o módulo $E_{p-i}^{(\infty)}$ por $F_{i}$, para $i=1, \ldots, p-1$, e o módulo $E_{q-i}^{(0)}$ por $G_{i}$, para $i=1, \ldots, q-1$. 
Com esta notação temos, para os simples regulares no tubo $\mathcal{T}_{\infty}^{\widetilde{\mathbb{A}}_{p, q}}$, que

$$
\begin{aligned}
& \tau F_{i}=\tau E_{p-i}^{(\infty)}=E_{p-i-1}^{(\infty)}=F_{i+1}, \text { para } 1 \leq i \leq p-2 \\
& \tau F_{p-1}=\tau E_{1}^{(\infty)}=E_{p}^{(\infty)} \\
& \tau E_{p}=F_{1}
\end{aligned}
$$

E para os simples regulares no tubo $\mathcal{T}_{0}^{\widetilde{\mathbb{A}}_{p, q}}$ temos que

$$
\begin{aligned}
\tau G_{i} & =\tau E_{q-i}^{(0)}=E_{q-i-1}^{(\infty)}=G_{i+1}, \text { para } 1 \leq i \leq q-2 \\
\tau G_{q-1} & =\tau E_{1}^{(0)}=E_{q}^{(0)} \\
\tau E_{q} & =G_{1} .
\end{aligned}
$$

Denotaremos as sequências ordenadas

$$
\begin{gathered}
\left(F_{1}, F_{2}, \ldots, F_{p-1}\right)=\left(S_{p-1}, S_{p-2}, \ldots, S_{1}\right)=F \\
\left(G_{1}, G_{2}, \ldots, G_{q-1}\right)=\left(S_{p+q-2}, S_{p+q-3}, \ldots, S_{p}\right)=G
\end{gathered}
$$

Afirmamos que $F$ e $G$ são sistemas estratificantes. De fato, $\operatorname{Hom}_{A}\left(F_{i}, F_{j}\right)=0$ para $i>j$, pois não existem morfismos entre módulos simples, e, para $i \geq j$,

$$
\operatorname{Ext}_{A}^{1}\left(F_{i}, F_{j}\right) \cong D \operatorname{Hom}_{A}\left(F_{j}, \tau F_{i}\right) \cong D \operatorname{Hom}_{A}\left(F_{j}, F_{i+1}\right)=0
$$

De forma análoga obtemos também que $G$ é um sistema estratificante.

Vamos denotar por $\mathcal{F}$ o conjunto $\left\{F_{1}, \ldots, F_{p-1}\right\}$ e por $\mathcal{G}$ o conjunto $\left\{G_{1}, \ldots, G_{q-1}\right\}$.

Adotaremos a seguinte convenção: dado um $A$-módulo $M$ escrevemos $\operatorname{Hom}_{A}(\mathcal{F}, M)=0$ significando que $\operatorname{Hom}_{A}(X, M)=0$, para todo elemento $X \in \mathcal{F}$.

Dado um conjunto de $A$-módulos regulares $\mathcal{X}$ denotaremos para cada $i \in \mathbb{Z}, i \neq 0$ por $\tau^{i}(\mathcal{X})$ o conjunto $\tau^{i}(\mathcal{X})=\left\{\tau^{i} X, X \in \mathcal{X}\right\}$, onde $\tau$ denota o transladado de Auslander-Reiten.

Usando as notações acima enunciamos o seguinte lema.

Lema 3.2.2. Sejam $\mathcal{F}$ e $\mathcal{G}$ os conjuntos definidos acima. Então:

1. (a) $\tau^{i}(\mathcal{F})=\left(\mathcal{F} \backslash\left\{F_{i}\right\}\right) \cup\left\{E_{p}^{(\infty)}\right\}$, para $1 \leq i \leq p-1$.

(b) $\tau^{-i}(\mathcal{F})=\left(\mathcal{F} \backslash\left\{F_{p-i}\right\}\right) \cup\left\{E_{p}^{(\infty)}\right\}$, para $1 \leq i \leq p-1$.

(c) $\tau^{p}(\mathcal{F})=\tau^{-p}(\mathcal{F})=\mathcal{F}$.

(d) Para cada $n \in \mathbb{N}, n \neq 0$, temos que

$$
\tau^{n}(\mathcal{F})=\left\{\begin{array}{l}
\mathcal{F}, \text { se } p \mid n \\
\tau^{r}(\mathcal{F}), \text { se } n \equiv r(\bmod p), \text { com } 1 \leq r \leq p-1
\end{array}\right.
$$




$$
\tau^{-n}(\mathcal{F})=\left\{\begin{array}{l}
\mathcal{F}, \text { se } p \mid n \\
\tau^{-r}(\mathcal{F}), \text { se } n \equiv r(\bmod p), \text { com } 1 \leq r \leq p-1
\end{array}\right.
$$

2. (a) $\tau^{i}(\mathcal{G})=\left(\mathcal{G} \backslash\left\{G_{i}\right\}\right) \cup\left\{E_{q}^{(0)}\right\}$, para $1 \leq i \leq q-1$.

(b) $\tau^{-i}(\mathcal{G})=\left(\mathcal{G} \backslash\left\{G_{q-i}\right\}\right) \cup\left\{E_{q}^{(0)}\right\}$, para $1 \leq i \leq q-1$.

(c) $\tau^{q}(\mathcal{G})=\tau^{-q}(\mathcal{G})=\mathcal{G}$.

(d) Para cada $n \in \mathbb{N}, n \neq 0$, temos que

$$
\begin{aligned}
\tau^{n}(\mathcal{G}) & =\left\{\begin{array}{l}
\mathcal{G}, \text { se } q \mid n \\
\tau^{r}(\mathcal{G}), \text { se } n \equiv r(\bmod q), \text { com } 1 \leq r \leq q-1
\end{array}\right. \\
\tau^{-n}(\mathcal{G}) & =\left\{\begin{array}{l}
\mathcal{G}, \text { se } q \mid n \\
\tau^{-r}(\mathcal{G}), \text { se } n \equiv r(\bmod q), \text { com } 1 \leq r \leq q-1
\end{array}\right.
\end{aligned}
$$

Demonstração. Vamos demonstrar 1 (a) por indução. Para $i=1$, pela definição, temos que $\tau(\mathcal{F})=\left\{\tau F_{1}, \ldots, \tau F_{p-1}\right\}$. Mas, por (3.3), temos que

$$
\tau(\mathcal{F})=\left\{F_{2}, \ldots, F_{p-1}, E_{p}^{\infty}\right\}=\left(\mathcal{F} \backslash\left\{F_{1}\right\}\right) \cup\left\{E_{p}^{(\infty)}\right\} .
$$

Suponhamos que a afirmação vale para todo $j$, com $1<j<p-1$. Isto é, suponhamos que $\tau^{i}(\mathcal{F})=\left(\mathcal{F} \backslash\left\{F_{i}\right\}\right) \cup\left\{E_{p}^{(\infty)}\right\}$, para $1<j<p-1$. Vamos calcular $\tau^{j+1}(\mathcal{F})$.

$$
\begin{aligned}
\tau^{j+1}(\mathcal{F}) & =\tau\left(\tau^{j}(\mathcal{F})\right) \\
& =\tau\left(\left(\mathcal{F} \backslash\left\{F_{j}\right\}\right) \cup\left\{E_{p}^{(\infty)}\right\}\right) \\
& =\tau\left(\mathcal{F} \backslash\left\{F_{j}\right\}\right) \cup \tau\left(\left\{E_{p}^{(\infty)}\right\}\right) \\
& \left.=\left[\left(\mathcal{F} \backslash\left\{F_{1}\right\}\right) \cup\left\{E_{p}^{(\infty)}\right\}\right) \backslash\left\{\tau F_{j}\right\}\right] \cup\left\{F_{1}\right\} \\
& =\left(\mathcal{F} \backslash\left\{F_{j+1}\right\}\right) \cup\left\{E_{p}^{(\infty)}\right\}
\end{aligned}
$$

Isto completa a prova de nossa afirmação.

A demonstração de 1(b) é análoga. A afirmação em 1(c) é clara, pois os elementos de $\mathcal{F}$ estão em tubo de posto $p$.

Vejamos $1(\mathrm{~d})$. Se $p \mid n$, então $n=k p$ para algum $k \geq 1$. Faremos indução sobre $k$. Por 1(c) temos que $\tau^{p} \mathcal{F}=\mathcal{F}$. Suponhamos que $\tau^{j p} \mathcal{F}=\mathcal{F}$, para todo $j$ tal que $1 \leq j<k$. Logo

$$
\begin{array}{rlrl}
\tau^{k p} \mathcal{F} & =\tau^{p}\left(\tau^{(k-1) p} \mathcal{F}\right) & \\
& =\tau^{p}(\mathcal{F}) & & \text { (pela hipótese) } \\
& =\mathcal{F} & & (\text { por } 1(\mathrm{c})) .
\end{array}
$$

Por outro lado, se $n \equiv r(\bmod p)$, com $1 \leq r \leq p-1$, então existe $k \geq 1$ tal que $n=k p+r$. Portanto $\tau^{(k p+r)}(\mathcal{F})=\tau^{r}\left(\tau^{k p}(\mathcal{F})\right)=\tau^{r}(\mathcal{F})$. 
Definição 3.2.3. Sejam A uma $K$-álgebra, $\left\{S_{1}, \ldots, S_{n}\right\}$ o conjunto de todos os A-módulos simples e dois a dois não isomorfos sobre $A$ e $M$ um A-módulo. $O$ suporte de $M$, que denotaremos por supp $M$, é o conjunto

$$
\operatorname{supp} M=\left\{i \in\{1, \ldots, n\}:\left[M: S_{i}\right] \neq 0\right\} .
$$

Dado um conjunto de A-módulos $\mathcal{X}$ o suporte de $\mathcal{X}$, que denotaremos por $\operatorname{Supp} \mathcal{X}$, é o conjunto

$$
\text { Supp } \mathcal{X}=\left\{i \in\{1, \ldots, n\}:\left[M: S_{i}\right] \neq 0, M \in \mathcal{X}\right\} \text {. }
$$

Reescrevendo o Lema 3.2.2 em termos do suporte dos conjuntos $\mathcal{F}$ e $\mathcal{G}$ temos o seguinte corolário.

Corolário 3.2.4. Seja $n \in \mathbb{N}, n \neq 0$. Então:

1. (a) Se $p \mid n$, então $\operatorname{Supp} \tau^{n} \mathcal{F}=\{1, \ldots, p-1\}=\operatorname{Supp} \mathcal{F}$.

(b) Se $n \equiv r(\bmod p) e 1 \leq r \leq p-1$, então

$$
\begin{aligned}
\operatorname{Supp} \tau^{n} \mathcal{F}=\tau^{r} \mathcal{F} & =\{0, \ldots, p+q-1\} \backslash\{p-r\} \\
\operatorname{Supp} \tau^{-n} \mathcal{F}=\tau^{-r} \mathcal{F} & =\{0, \ldots, p+q-1\} \backslash\{r\} .
\end{aligned}
$$

2. (a) Se $q \mid n$, então $\operatorname{Supp} \tau^{n} \mathcal{G}=\mathcal{G}=\{p, \ldots, p+q-2\}$.

(b) Se $n \equiv r(\bmod q) e 1 \leq r \leq q-1$, então

$$
\begin{aligned}
\operatorname{Supp} \tau^{n} \mathcal{G}=\tau^{r} \mathcal{G} & =\{0, \ldots, p+q-1\} \backslash\{p+q-r-1\} \\
\operatorname{Supp} \tau^{-n} \mathcal{G}=\tau^{-r} \mathcal{G} & =\{0, \ldots, p+q-1\} \backslash\{p+r-1\} .
\end{aligned}
$$

Observação 3.2.5. Como as componentes regulares sobre uma álgebra hereditária euclideana são duas a duas ortogonais (Ver Teorema 3.1.9) e os sistemas estratificantes $F$ e $G$ estão sobre duas componentes diferentes, então $(F, G)$ e $(G, F)$ são também sistemas estratificantes.

Na sequência, vamos completar $(F, G)$ encontrando todos os possíveis módulos $X$ e $Y$ de tal forma que $(X, F, G, Y)$ seja um sistema estratificante completo. Para isto, procuramos inicialmente todos os possíveis módulos pós-projetivos de tal forma que $(F, G, Y)$ seja um sistema estratificante.

Usando a Proposição 3.1.7 podemos afirmar que o módulo $Y$ tem que ser ou pós-projetivo ou pré-injetivo. Vamos procurar primeiro os módulos pós-projetivos tais que $(X, F, G, Y)$ é um sistema estratificante.

Antes de fazer tal busca, enunciamos alguns resultados básicos que usaremos com frequência. 
Lema 3.2.6. Seja A uma álgebra hereditária. São válidas as seguintes afirmaçôes:

1. Se $P_{j}$ e $P_{m}$ são A-módulos projetivos indecomponíveis quaisquer, então

$$
\operatorname{Ext}_{A}^{1}\left(\tau^{-t} P_{j}, \tau^{-t-r} P_{m}\right)=0, \text { para todo } t, r \geq 0
$$

2. Se $I_{j}$ e $I_{m}$ são A-módulos injetivos indecomponíveis quaisquer, então

$$
\operatorname{Ext}_{A}^{1}\left(\tau^{t} I_{j}, \tau^{t-r} I_{m}\right)=0, \text { para todo } t \geq r \geq 0
$$

Demonstração. Para demonstrar 1. usamos a fórmula de Auslander e obtemos que

$$
\begin{aligned}
\operatorname{Ext}_{A}^{1}\left(\tau^{-t} P_{j}, \tau^{-t-r} P_{m}\right) & \cong D \operatorname{Hom}_{A}\left(\tau^{-t-r} P_{m}, \tau^{-t+1} P_{j}\right) \\
& \cong D \operatorname{Hom}_{A}\left(\tau^{-r-1} P_{m}, P_{j}\right)
\end{aligned}
$$

Mas $\operatorname{Hom}_{A}\left(\tau^{-r-1} P_{m}, P_{j}\right)=0$, pois caso contrário $P_{j}$ teria um predecessor não projetivo em $\Gamma(\bmod A)$, o que não acontece em uma álgebra hereditária. Portanto

$$
\operatorname{Ext}_{A}^{1}\left(\tau^{-t} P_{j}, \tau^{-t-r} P_{m}\right)=0
$$

Para a afirmação 2. usamos de novo a fórmula de Auslander e obtemos que

$$
\begin{aligned}
\operatorname{Ext}_{A}^{1}\left(\tau^{t} I_{j}, \tau^{t-r} I_{m}\right) & \cong D \operatorname{Hom}_{A}\left(\tau^{t-r} I_{m}, \tau^{t+1} I_{j}\right) \\
& \cong \operatorname{Hom}_{A}\left(I_{m}, \tau^{r+1} I_{j}\right)
\end{aligned}
$$

Mas $\operatorname{Hom}_{A}\left(I_{m}, \tau^{r+1} I_{j}\right)=0$, pois caso contrário $I_{m}$ teria um sucessor não injetivo em $\Gamma(\bmod A)$. Portanto

$$
\operatorname{Ext}_{A}^{1}\left(\tau^{t} I_{j}, \tau^{t-r} I_{m}\right)=0
$$

Proposição 3.2.7. Se Y é A-módulo pós-projetivo tal que $(F, G, Y)$ é um sistema estratificante, então $Y$ é um dos módulos na seguinte lista:

1. $P_{0}$.

2. $P_{p+q-1}$.

3. $\tau^{-t} P_{0}$, com $t \geq 1$ tal que $p \mid t$ e $q \mid t$.

4. $\tau^{-t} P_{p+q-1}$,com $t \geq 1$ tal que $p \mid t$ e $q \mid t$.

5. $\tau^{-t} P_{p-r}$, com $t \geq 1$ tal que $q \mid t, t \equiv r(\bmod p) e 1 \leq r \leq p-1$.

6. $\tau^{-t} P_{p+q-r-1}$, com $t \geq 1$ tal que $p \mid t, t \equiv r(\bmod q)$ e $1 \leq r \leq q-1$. 
Demonstração. Se $M$ é um $A$-módulo regular então, pela fórmula de Auslander,

$$
\operatorname{Ext}_{A}^{1}\left(\tau^{-t} P_{j}, M\right) \cong D \operatorname{Hom}_{A}\left(\tau^{-1} M, \tau^{-t} P_{j}\right), \text { para } t \geq 0 .
$$

Mas $\operatorname{Hom}_{A}\left(\tau^{-1} M, \tau^{-t} P_{j}\right)=0$ pois, segundo Corolário 2.13 do Cap. VIII em [2], não existem morfismos não nulos de módulos regulares a módulos pós-projetivos. Portanto

$$
\operatorname{Ext}_{A}^{1}\left(\tau^{-t} P_{j}, M\right)=0 \text {, para } t \geq 0 .
$$

Assim para verificar que $\left(F, G, \tau^{-t} P_{j}\right)$ é um sistema estratificante é suficiente mostrar que

$$
\operatorname{Hom}_{A}\left(\tau^{-t} P_{j}, \mathcal{F}\right)=0=\operatorname{Hom}_{A}\left(\tau^{-t} P_{j}, \mathcal{G}\right) .
$$

Por outro lado, pelo Corolário 2.15 Cap. IV em [2], temos que

$$
\operatorname{Hom}_{A}\left(\tau^{-t} P_{j}, \mathcal{F}\right) \cong \operatorname{Hom}_{A}\left(P_{j}, \tau^{t} \mathcal{F}\right) \text { e que } \operatorname{Hom}_{A}\left(\tau^{-t} P_{j}, \mathcal{G}\right) \cong \operatorname{Hom}_{A}\left(P_{j}, \tau^{t} \mathcal{G}\right) \text {. }
$$

Além disso, pelo Corolário 1.5.5, para um $A$-módulo $M$ vale que

$$
\operatorname{Hom}_{A}\left(P_{j}, M\right)=0 \text { se, e somente se, } j \notin \operatorname{supp} M \text {. }
$$

Assim,

$$
\begin{gathered}
\operatorname{Hom}_{A}\left(P_{j}, \tau^{t} \mathcal{F}\right)=0 \text { se, e somente se, } j \notin \operatorname{Supp} \tau^{t} \mathcal{F} \text { e } \\
\operatorname{Hom}_{A}\left(P_{j}, \tau^{t} \mathcal{G}\right)=0 \text { se, e somente se, } j \notin \operatorname{Supp} \tau^{t} \mathcal{G} .
\end{gathered}
$$

Portanto, podemos afirmar que

$$
\left(F, G, \tau^{-t} P_{j}\right) \text { é um sistema estratificante } \Leftrightarrow j \in\left(\operatorname{Supp} \tau^{t} \mathcal{G}\right)^{\prime} \cap\left(\operatorname{Supp} \tau^{t} \mathcal{F}\right)^{\prime},
$$

onde $\left(\operatorname{Supp} \tau^{t} \mathcal{G}\right)^{\prime}$ denota o complemento de $\left(\operatorname{Supp} \tau^{t} \mathcal{G}\right)$ com relação ao conjunto $\{1, \ldots, p+q-1\}$.

Tendo em vista esta última afirmação vamos encontrar os sistemas estratificantes da forma $(F, G, Y)$, com $Y \cong \tau^{-t} P_{j}$, considerando as distintas possibilidades para o inteiro $t$.

Caso 1. Se $t=0$, então $Y \cong P_{j}$ e $\left(F, G, P_{j}\right)$ é um sistema estratificante se, e somente se, $j \in(\operatorname{Supp} \mathcal{F})^{\prime} \cap(\operatorname{Supp} \mathcal{G})^{\prime}$.

Mas, $\operatorname{Supp} \mathcal{F}=\{1, \ldots, p-1\}$ e $\operatorname{Supp} \mathcal{G}=\{p, \ldots, p+q-2\}$. Então, $\left(F, G, P_{j}\right)$ é um sistema estratificante se, e somente se, $j=0$ ou $j=p+q-1$.

Caso 2. Se $t$ é tal que $p \mid t$ e $q \mid t$. Então, pelo Corolário 3.2.4, 1.(a) e 2.(a), temos que

$$
\operatorname{Supp} \tau^{t} \mathcal{F}=\operatorname{Supp} \mathcal{F} \text { e } \operatorname{Supp} \tau^{t} \mathcal{G}=\operatorname{Supp} \mathcal{G}
$$

Assim este caso se reduz ao caso anterior. Ou seja $\left(F, G, \tau^{-t} P_{j}\right)$, onde $p \mid t$ e $q \mid t$, é um sistema 
estratificante se, e somente se, $j=0$ ou $j=p+q-1$.

Caso 3. Se $t$ é tal que $q \mid t$ e $t \equiv r(\bmod p)$, com $1 \leq r \leq p-1$, então pelo Corolário 3.2.4, 1.(b) e 2.(a), temos que

$$
\begin{gathered}
\left(\operatorname{Supp} \tau^{t} \mathcal{F}\right)^{\prime}=\left(\operatorname{Supp} \tau^{r} \mathcal{F}\right)^{\prime}=\{p-r\} \text { e } \\
\left(\operatorname{Supp} \tau^{t} \mathcal{G}\right)^{\prime}=(\operatorname{Supp} \mathcal{G})^{\prime}=\{0, \ldots, p-1\} \cup\{p+q-1\} .
\end{gathered}
$$

Por tanto $\left(F, G, \tau^{-t} P_{j}\right)$ é um sistema estratificante se, e somente se, $j=p-r$.

Caso 4. Se $t$ é tal que $p \mid t$ e $q \backslash\langle t$. De forma análoga ao caso anterior podemos concluir que, sob estas hipóteses, $\left(F, G, \tau^{-t} P_{j}\right)$ é um sistema estratificante se, e somente se, $j=p+q-r-1$, onde $r$ é tal que $t \equiv r(\bmod q)$ e $1 \leq r \leq q-1$.

Caso 5. Se $t \equiv r_{1}(\bmod p)$ e $t \equiv r_{2}(\bmod q)$, com $0<r_{1}<p$ e $0<r_{2}<q$. Então usando argumentos análogos aos casos anteriores, basta analisar o conjunto (Supp $\left.\tau^{r_{1}} \mathcal{F}\right)^{\prime} \cap\left(\operatorname{Supp} \tau^{r_{2}} \mathcal{G}\right)^{\prime}$.

Mas segundo Lema 3.2.4, temos que

$$
\left(\operatorname{Supp} \tau^{r_{1}} \mathcal{F}\right)^{\prime} \cap\left(\operatorname{Supp} \tau^{r_{2}} \mathcal{G}\right)^{\prime}=\left\{p-r_{1}\right\} \cap\left\{p+q-r_{2}-1\right\}=\phi .
$$

Portanto não existe um sistema estratificante neste caso.

Dessa forma $Y$ é um dos módulos da lista apresentada.

Analisemos agora o caso em que $Y$ é pré-injetivo, que resulta na seguinte proposição.

Proposição 3.2.8. Se $Y$ é A-módulo pré-injetivo tal que $(F, G, Y)$ é um sistema estratificante. Então $Y$ está na seguinte lista:

1. $\tau^{t} I_{p}$, com $t \geq 1$ tal que $t \equiv p-1(\bmod p)$ e $q \mid t$.

2. $\tau^{t} I_{1}$, com $t \geq 1$ tal que $t \equiv q-1(\bmod q)$ e $p \mid t$.

3. $\tau^{t} I_{0}$, com $t \geq 1$ tal que $t \equiv p-1(\bmod p)$ e $t \equiv q-1(\bmod q)$.

4. $\tau^{t} I_{p+q-1}$, com $t \geq 1$ tal que $t \equiv p-1(\bmod p)$ et $t \equiv q-1(\bmod q)$.

5. $\tau^{t} I_{r+1}$, com $t \geq 1$ tal que $t \equiv r(\bmod p), r \neq p-1$ e $t \equiv q-1(\bmod q)$.

6. $\tau^{t} I_{p+r}$, com $t \geq 1$ tal que $t \equiv r(\bmod q), r \neq q-1$ e $t \equiv p-1(\bmod p)$.

Demonstração. Seja $Y$ um $A$-módulo pré-injetivo. Então $Y \cong \tau^{k} I_{j}$, para algum $k \geq 0$ e algum $j \in\{0,1, \ldots, p, \ldots, p+q-1\}$. Notemos que, pelo Corolário 2.13 (VIII) em [2], se $M$ é um $A$-módulo regular e $Y$ é um $A$-módulo pré-injetivo, então $\operatorname{Hom}_{A}(Y, M)=0$. Por outro lado, pela fórmula de Auslander, temos que para $k \geq 1$

$$
\operatorname{Ext}_{A}^{1}\left(\tau^{k} I_{j}, M\right) \cong D \operatorname{Hom}_{A}\left(\tau^{-1} M, \tau^{k} I_{j}\right) \cong D \operatorname{Hom}_{A}\left(\tau^{-(k+1)} M, I_{j}\right)
$$


Além disso, usando o isomorfismo acima e Corolário 1.5.5, temos que

$$
\operatorname{Ext}_{A}^{1}\left(\tau^{k} I_{j}, M\right)=0 \text { se, e somente se, } j \notin \operatorname{supp} \tau^{-(k+1)} M \text {. }
$$

Das afirmações anteriores podemos concluir que

$$
\left(F, G, \tau^{k} I_{j}\right) \text { é um sistema estratificante } \Leftrightarrow j \notin \operatorname{Supp} \tau^{-(k+1)} \mathcal{F} \cup \operatorname{Supp} \tau^{-(k+1)} \mathcal{G} \text {. }
$$

Usaremos esta afirmação para encontrar todos os sistemas estratificantes da forma $(F, G, Y)$ com $Y$ pré-injetivo.

Caso a. Suponhamos que $Y \cong I_{j}$, para algum $j \in\{0,1, \ldots, p, \ldots, p+q-1\}$, ou seja, $Y$ é um injetivo indecomponível. Pelo Lema 3.2.4, temos que

$$
\left(\operatorname{Supp} \tau^{-1} \mathcal{F}\right)^{\prime}=\{1\} \text { e que }\left(\operatorname{Supp} \tau^{-1} \mathcal{G}\right)^{\prime}=\{p\}
$$

Então Supp $\tau^{-1} \mathcal{F} \cup \operatorname{Supp} \tau^{-1} \mathcal{G}=\{0, \ldots, p+q-1\}$. Portanto não existe um $j$ tal que $\left(F, G, I_{j}\right)$ seja um sistema estratificante.

Caso b. Suponhamos que $Y \cong \tau^{t} I_{j}$, com $t \geq 1$ e que $p \mid t$ e $q \mid t$. Logo

$$
\operatorname{Supp} \tau^{-(t+1)} \mathcal{F}=\operatorname{Supp} \tau^{-1} \mathcal{F} \text { e } \operatorname{Supp} \tau^{-(t+1)} \mathcal{G}=\operatorname{Supp} \tau^{-1} \mathcal{G}
$$

Assim este caso se reduz ao anterior e, então não existe um $j$ tal que $\left(F, G, \tau^{t} I_{j}\right)$ seja um sistema estratificante, nas condições em que $p \mid t$ e $q \mid t$.

Caso c. Suponhamos que $Y \cong \tau^{t} I_{j}$, com $t$ tal que $p \not t$ e $q \mid t$. Seja $t \equiv r(\bmod p), 1 \leq r \leq p-1$. Devemos considerar dois casos: quando $r=p-1$ e quando $r \neq p-1$.

- Se $r=p-1$, então

$$
\operatorname{Supp} \tau^{-(t+1)} \mathcal{F}=\operatorname{Supp} \tau^{-(r+1)} \mathcal{F}=\operatorname{Supp} \tau^{-p} \mathcal{F}=\operatorname{Supp} \mathcal{F}
$$

Por outro lado, pelo Corolário 3.2.4, 2.b, temos

$$
\operatorname{Supp} \tau^{-(t+1)} \mathcal{G}=\operatorname{Supp} \tau^{-1} \mathcal{G}=\{0, \ldots, p+q-1\} \backslash\{p\} .
$$

Assim,

$$
\operatorname{Supp} \tau^{-(t+1)} \mathcal{F} \cup \operatorname{Supp} \tau^{-(t+1)} \mathcal{G}=\{0, \ldots, p+q-1\} \backslash\{p\} .
$$

Portanto $\left(F, G, \tau^{t} I_{p}\right)$ é um sistema estratificante, é o caso 1 da lista.

- Se $r \neq p-1$, temos que

$$
\operatorname{Supp} \tau^{-(t+1)} \mathcal{F}=\operatorname{Supp} \tau^{-(r+1)} \mathcal{F}=\{0, \ldots, p+q-1\} \backslash\{r+1\} .
$$


Então Supp $\tau^{-(t+1)} \cup \operatorname{Supp} \tau^{-(t+1)} \mathcal{G}=\{0, \ldots, p+q-1\}$ e, portanto, não existe um $j$ tal que $\left(F, G, \tau^{t} I_{j}\right)$ seja um sistema estratificante.

Caso d. Suponhamos que $Y \cong \tau^{t} I_{j}$, com $t \geq 1$ tal que $q \backslash t$ e que $p \mid t$. Seja $r \equiv t(\bmod q)$, $1 \leq r \leq q-1$. Devemos considerar aqui também dois casos: quando $r=q-1$ e quando $r \neq q-1$.

- Se $r=q-1$, então

$$
\operatorname{Supp} \tau^{-(t+1)} \mathcal{G}=\operatorname{Supp} \tau^{-(r+1)} \mathcal{G}=\operatorname{Supp} \tau^{-q} \mathcal{G}=\operatorname{Supp} \mathcal{G}
$$

Por outro lado,

$$
\operatorname{Supp} \tau^{-(t+1)} \mathcal{F}=\operatorname{Supp} \tau^{-1} \mathcal{F}=\{0, \ldots, p+q-1\} \backslash\{1\}
$$

Assim,

$$
\operatorname{Supp} \tau^{-(t+1)} \mathcal{F} \cup \operatorname{Supp} \tau^{-(t+1)} \mathcal{G}=\{0, \ldots, p+q-1\} \backslash\{1\} .
$$

Por tanto $\left(F, G, \tau^{t} I_{1}\right)$ é um sistema estratificante, que é o caso 2 da lista.

- Se $r \neq q-1$, então $1 \leq r \leq q-2$, temos pois que

$$
\operatorname{Supp} \tau^{-(t+1)} \mathcal{G}=\operatorname{Supp} \tau^{-(r+1)} \mathcal{G}=\{0, \ldots p+q-1\} \backslash\{p+r\} .
$$

Então Supp $\tau^{-(t+1)} \mathcal{F} \cup \operatorname{Supp} \tau^{-(t+1)} \mathcal{G}=\{0, \ldots, p+q-1\}$ e, por tanto, não existe um $j$ tal que $\left(F, G, \tau^{t} I_{j}\right)$ seja um sistema estratificante.

Caso e. Suponhamos que $Y \cong \tau^{t} I_{j}$, com $t$ tal que $q \Lambda t$ e que $p \bigwedge t$. Sejam $r_{1}$ e $r_{2}$ tais que $t \equiv r_{1}(\bmod p), t \equiv r_{2}(\bmod q), 1 \leq r_{1} \leq p-1$ e $1 \leq r_{2} \leq q-1$. Vamos considerar varias situações.

- Sejam $r_{1}=p-1$ e $r_{2}=q-1$. Temos que:

$$
\operatorname{Supp} \tau^{-(t+1)} \mathcal{F} \cup \operatorname{Supp} \tau^{-(t+1)} \mathcal{G}=\operatorname{Supp} \mathcal{F} \cup \operatorname{Supp} \mathcal{G}=\{1, \ldots, p+q-2\}
$$

Por tanto, neste caso, os possíveis sistemas estratificantes são $\left(F, G, \tau^{t} I_{0}\right)$ e $\left(F, G, \tau^{t} I_{p+q-1}\right)$, que constituem os casos 3 e 4 da lista.

- Sejam $r_{2}=q-1$ e $r_{1} \neq p-1$. Então temos que

$$
\begin{gathered}
\operatorname{Supp} \tau^{-(t+1)} \mathcal{G}=\operatorname{Supp} \mathcal{G} \text { e } \\
\operatorname{Supp} \tau^{-(t+1)} \mathcal{F}=\operatorname{Supp} \tau^{-\left(r_{1}+1\right)} \mathcal{F}=\{0, \ldots, p+q-1\} \backslash\left\{r_{1}+1\right\} .
\end{gathered}
$$

Logo $\left(F, G, \tau^{t} I_{r_{1}+1}\right)$ é um sistema estratificante originando o caso 5 da lista. 
- Suponhamos que $r_{1}=p-1$ e que $r_{2} \neq q-1$. Neste caso temos que

$$
\begin{gathered}
\operatorname{Supp} \tau^{-(t+1)} \mathcal{F}=\operatorname{Supp} \mathcal{F} \text { e } \\
\operatorname{Supp} \tau^{-(t+1)} \mathcal{G}=\operatorname{Supp} \tau^{-\left(r_{2}+1\right)} \mathcal{G}=\{0, \ldots p+q-1\} \backslash\left\{p+r_{2}\right\} .
\end{gathered}
$$

Logo $\left(F, G, \tau^{t} I_{p+r_{2}}\right)$ é um sistema estratificante, dando origem ao caso 6 da lista.

- Se $r_{1} \neq p-1$ e $r_{2} \neq q-1$, neste caso temos que:

$$
\begin{aligned}
& \operatorname{Supp} \tau^{-(t+1)} \mathcal{G}=\operatorname{Supp} \tau^{-\left(r_{2}+1\right)} \mathcal{G}=\{0, \ldots p+q-1\} \backslash\left\{p+r_{2}\right\} \text { e } \\
& \operatorname{Supp} \tau^{-(t+1)} \mathcal{F}=\operatorname{Supp} \tau^{-\left(r_{1}+1\right)} \mathcal{F}=\{0, \ldots, p+q-1\} \backslash\left\{r_{1}+1\right\}
\end{aligned}
$$

Portanto não existe $j$ tal que $\left(F, G, \tau^{t} I_{j}\right)$ seja um sistema estratificante.

Lema 3.2.9. Sejam A uma K-álgebra e

$$
0 \longrightarrow L \stackrel{\left(f^{\prime} g^{\prime}\right)^{t}}{\longrightarrow} X \oplus Y \stackrel{(f g)}{\longrightarrow} M \longrightarrow 0
$$

uma sequência quase-cindida em $\bmod A$. Então:

1. Se $f^{\prime}$ e $g^{\prime}$ são monomorfismos, então $f$ e $g$ são monomorfismos.

2. Se $f^{\prime}$ e $g^{\prime}$ são epimorfismos, então $f$ e $g$ são epimorfismos.

Demonstração. Vamos demonstrar 1, pois 2 é análoga. Como $(*)$ é uma sequência quasecindida se $f^{\prime}$ e $g^{\prime}$ são morfismos irredutíveis, então são ou monomorfismos próprios ou epimorfismos próprios. Portanto se $f^{\prime}$ e $g^{\prime}$ são monomorfismos, então são monomorfismos próprios. Então temos que $\ell(L)<\ell(X)$ e que $\ell(L)<\ell(Y)$.

Por outro lado, como $(*)$ é uma sequência exata, temos que $\ell(L)+\ell(M)=\ell(X)+\ell(Y)$. Logo, $\ell(M)=\ell(X)+(\ell(Y)-\ell(L))$. Mas $\ell(Y)-\ell(L)>0$, portanto $\ell(M)>\ell(X)$.

O morfismo $f: X \longrightarrow M$ também é ou monomorfismo próprio ou epimorfismo próprio, por ser irredutível. Como $\ell(M)>\ell(X)$ então $f$ tem que ser monomorfismo. De forma análoga se demonstra que $g$ deve ser monomorfismo.

Para continuar o trabalho será muito útil conhecer os fatores de composição dos $A$-módulos pós-projetivos sobre a álgebra $K \triangle\left(\widetilde{\mathbb{A}}_{p, q}\right)$, justamente isto o que estudamos na proposição que se segue.

Proposição 3.2.10. Seja $A$ a álgebra $K \triangle\left(\widetilde{\mathbb{A}}_{p, q}\right)$. Então a componente pós-projetiva de $\Gamma(\bmod A)$ tem as seguintes propriedades: 
1. Todos os morfismos na componente pós-projetiva de $\Gamma\left(\bmod \triangle\left(\widetilde{\mathbb{A}}_{p, q}\right)\right)$ são monomorfismos.

2. O módulo $P_{p+q-1}$ e todos seus sucessores são módulos sinceros.

3. O minimo inteiro $r$ com a propriedade de que todos os módulos da forma $\tau^{-r} P_{i}$ com $i \in\{0, \ldots, p+q-1\}$ são sinceros é $p$.

4. Se $0 \leq i \leq p-1$, então

- O menor inteiro $r$ tal que $\tau^{-r} P_{i}$ é um módulo sincero é $r=p-i$. Além disso, todos os A-módulos da forma $\tau^{-k} P_{i}$, com $k>p-i$, são A-módulos sinceros.

- Se $0<k<p-i$, então os fatores de composiçãa de $\tau^{-k} P_{i}$ são os A-módulos simples $S_{j} \operatorname{com} 0 \leq j \leq i+k$ e $p \leq j \leq p+k-1$.

- Os fatores de composição do projetivo $P_{i}$ são os simples $S_{j}$ com $0 \leq j \leq i$.

5. Se $p \leq i \leq p+q-2$, então:

- se $i \leq q-1$, o menor inteiro $r$ tal que $\tau^{-r} P_{i}$ é sincero é $r=p$. Além disso, todos os A-módulos da forma $\tau^{-k} P_{i}$ com $k>p$ são sinceros.

- se $q-1 \leq i \leq p+q-1$, o menor inteiro tal que $\tau^{-r} P_{i}$ é sincero é $r=p+q-1-i$. Ademais, todos os A-módulos da forma $\tau^{-k} P_{i}$ com $k>p+q-1-i$ são sinceros.

- se $\tau^{-k} P_{i}$ não é um A-módulo sincero, seus fatores de composição são os A-módulos simples $S_{j}$ com $p \leq j \leq i+k$ e $0 \leq j \leq k$.

6. Se $1 \leq k \leq p-1$, então os fatores de composição de $\tau^{-k} P_{0}$ são os A-módulos simples $S_{j}$ com $0 \leq j \leq k$ e $p \leq j \leq p-1+k$.

Demonstração. Para a demonstração desta proposição usamos a estrutura da componente pós-projetiva de $\Gamma(\bmod A)$, que desenhamos abaixo

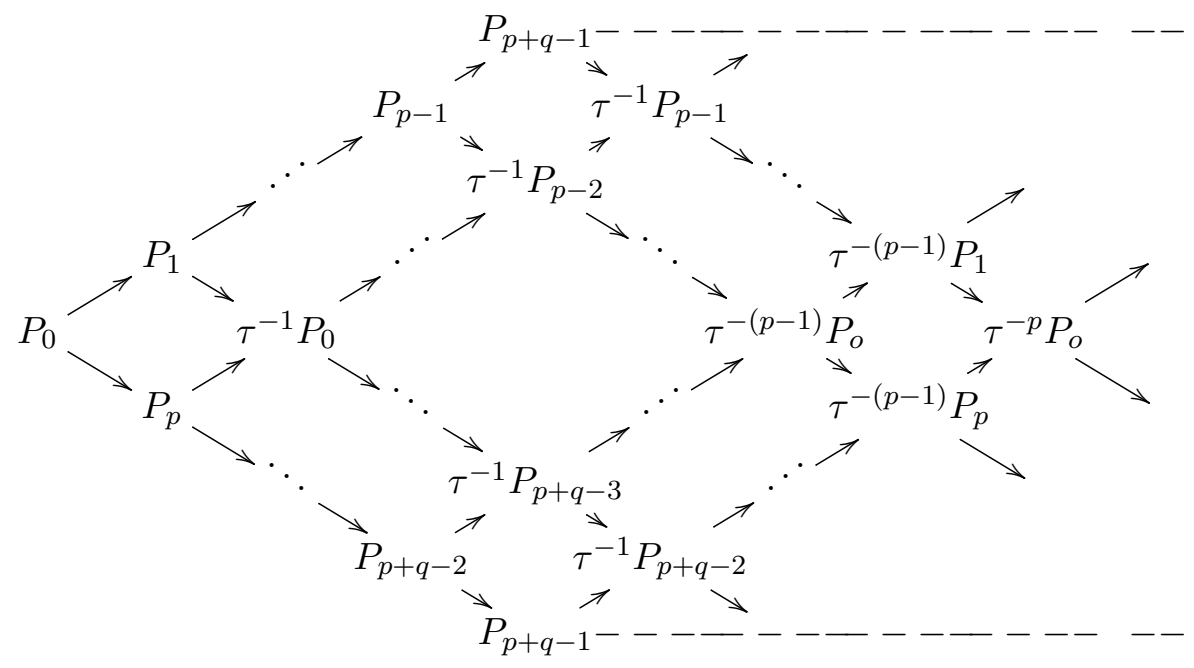


Sistemas estratificantes sobre álgebras de tipo $\Delta\left(\widetilde{\mathbb{A}}_{p, q}\right)$

Para provar 1, observemos que na sequência quase-cindida

$$
0 \longrightarrow P_{0} \stackrel{\left(\alpha^{\prime} \beta^{\prime}\right)^{t}}{\longrightarrow} P_{1} \oplus P_{p} \stackrel{(\alpha \beta)}{\longrightarrow} \tau^{-1} P_{0} \longrightarrow 0
$$

os morfismos $\alpha^{\prime}$ e $\beta^{\prime}$ são monomorfismos, pois são morfismos irredutíveis entre módulos projetivos. Então, segundo o Lema 3.2.9, $\alpha$ e $\beta$ também são monomorfismos. De forma análoga, nas sequências quase-cindidas sobre $A$ começando num projetivo as flechas representam monomorfismos. Por isto, de acordo com a forma da componente pós-projetiva de $\Gamma(\bmod A)$ todos os morfismos nesta componente representam monomorfismos.

Para 2 , é suficiente ver que, em $\Gamma(\bmod A)$, existem os caminhos

$$
P_{0} \longrightarrow P_{1} \longrightarrow \cdots \longrightarrow P_{p-1} \longrightarrow P_{p+q-1} \text { e } P_{0} \longrightarrow P_{p} \longrightarrow \cdots \longrightarrow P_{p+q-1} \longrightarrow P_{p+q-1},
$$

onde, segundo 1, as fechas representam monomorfismos. Portanto, segundo o Corolário 1.5.5, $\left[P_{p+q-1}: S_{i}\right] \neq 0$, para $i=0,1, \ldots, p+q-1$, isto é $P_{p+q-1}$ é um $A$-módulo sincero. Uma vez que $P_{p+q-1}$ é sincero segue, de 1 , que todos os seus sucessores são também módulos sinceros.

Para a afirmação 3 , observemos que nenhum dos antecessores em $\Gamma(\bmod A)$ dos módulos que aparecem nos caminhos abaixo

$$
\begin{gathered}
P_{p+q-1} \longrightarrow \tau^{-1} P_{p-1} \longrightarrow \tau^{-2} P_{p-2} \longrightarrow \cdots \longrightarrow \tau^{-(p-1)} P_{0} \longrightarrow \tau^{-p} P_{0} \\
P_{p+q-1} \longrightarrow \tau^{-1} P_{p+q-2} \longrightarrow \tau^{-2} P_{p+q-3} \longrightarrow \cdots \longrightarrow \tau^{-(p-1)} P_{p} \longrightarrow \tau^{-p} P_{0}
\end{gathered}
$$

têm $S_{p+q-1}$ como fator de composição. Em particular, $\tau^{-(p-1)} P_{0}$ não tem $S_{p+q-1}$ como fator de composição. Portanto, de 1, temos que nenhum dos seus antecessores o têm. Mas, por outro lado, qualquer sucessor de $\tau^{-(p-1)} P_{0}$, e de qualquer dos módulos em (3.7) e (3.8), é sincero.

Para demonstrar 4, notemos que todos os módulos da forma $\tau^{-(p-i)} P_{i}$, com $0 \leq i \leq p-1$, estão no caminho (3.7) e, portanto, são módulos sinceros. Além disso, como dito anteriormente, qualquer predecessor de algum dos módulos que aparecem em (3.7) não tem $S_{p+q-1}$ como fator de composição. Deste modo, se $0 \leq i \leq p-1$ nenhum dos módulos da forma $\tau^{-k} P_{i}$, com $k<p-i$ é sincero.

Por outro lado, notemos que os predecessores de $P_{i}$, com $0 \leq i \leq p-1$, são os projetivos $P_{0}, P_{1}, \ldots, P_{i-1}$. Portanto, os fatores de composição de $P_{i}$, com $0 \leq i \leq p-1$, são os simples $S_{j}$ com $0 \leq j \leq i$. De forma análoga, para $p \leq i<p+q-1$, os fatores de composição de $P_{i}$ são $S_{j}, \operatorname{com} p \leq j \leq i$ e $j=0$.

Seja $0 \leq i \leq p-1$. Então em $\Gamma(\bmod A)$ existem os seguintes caminhos

$$
\begin{aligned}
& P_{i+k} \longrightarrow \tau^{-1} P_{i+k-1} \longrightarrow \tau^{-2} P_{i+k-2} \longrightarrow \cdots \longrightarrow \tau^{-(k-1)} P_{i+1} \longrightarrow \tau^{-k} P_{i} \\
& P_{p+k-1} \longrightarrow \tau^{-1} P_{p+k-2} \longrightarrow \tau^{-2} P_{p+k-3} \longrightarrow \cdots \longrightarrow \tau^{-(k-1)} P_{p} \longrightarrow \tau^{-k} P_{0} \longrightarrow \\
& \tau^{-k} P_{1} \longrightarrow \tau^{-k} P_{2} \longrightarrow \cdots \longrightarrow \tau^{-k} P_{i}
\end{aligned}
$$


Além disso, não existem caminhos de $P_{j}$, com $j \geq i+k$, para $\tau^{-k} P_{i}$. Portanto, os fatores de composição de $\tau^{-k} P_{i}$, exceto multiplicidades, são os fatores de composição de $P_{i+k}$ e os de $P_{p+k-1}$. Isto é, os fatores de composição de $\tau^{-k} P_{i}$ são $S_{j}$ com $0 \leq j \leq i$ e $p \leq i<p+q-1$.

A parte 5 é demonstrada de forma análoga à anterior.

Proposição 3.2.11. Seja $A=\triangle\left(\widetilde{\mathbb{A}}_{p, q}\right)$. A lista completa dos sistemas estratificantes completos da forma $(X, F, G, Y)$, onde $Y$ é um A-módulo pós-projetivo é a seguinte:

1. $\left(S_{p+q-1}, F, G, P_{0}\right)$.

2. $\left(X, F, G, P_{p+q-1}\right)$, onde

$$
X \cong\left\{\begin{array}{l}
\tau^{-p+1} P_{0}, \text { se } p=q \\
\tau^{-p+1} P_{q-1}, \text { se } p \neq q
\end{array}\right.
$$

3. $\left(X, F, G, \tau^{-t} P_{p+q-1}\right)$ com $t \in \mathbb{N}$ tal que $p \mid t$ e $q \mid t$, onde

$$
X \cong\left\{\begin{array}{l}
\tau^{-t-p+1} P_{0}, \text { se } p=q \\
\tau^{-t-p+1} P_{q-1}, \text { se } p \neq q
\end{array}\right.
$$

4. $\left(\tau^{-t+1} P_{p+q-1}, F, G, \tau^{-t} P_{0}\right)$, com $t \in \mathbb{N}$ tal que $p \mid t$ e $q \mid t$.

5. $\left(\tau^{-t-(p-r-1)} P_{q+r-1}, F, G, \tau^{-t} P_{p-r}\right)$, com $t, r \in \mathbb{N}$ tais que $q \mid t, t \equiv r(\bmod p)$ e $1 \leq r \leq$ $p-1$.

6. $\left(X, F, G, \tau^{-t} P_{p+q-r-1}\right)$, com $t, r \in \mathbb{N}$ tais que $p \mid t, t \equiv r(\bmod q) e 1 \leq r \leq q-1$, onde

$$
X \cong\left\{\begin{array}{l}
\tau^{-t-q+r+1} P_{p-q+r}, \text { se } p \leq q-r \\
\tau^{-t-p+1} P_{q-r-1}, \text { se } p>q-r
\end{array}\right.
$$

Demonstração. A Proposição 3.2.7 caracteriza os sistemas estratificantes da forma $(F, G, Y)$, com $Y$ um $A$-módulo pós-projetivo. Usando tal caracteização obtemos os sistemas estratificantes do tipó $(X, F, G, Y)$.

Em virtude dos Lemas 2.3.16 e 2.3.17, como $\triangle\left(\widetilde{\mathbb{A}}_{p, q}\right)$ tem $p+q$ vértices, o módulo $X$ tal que a sequência $\left(X, F, G, \tau^{-t} P_{l}\right)$ é um sistema estratificante completo é único. Portanto a demonstração consistirá em verificar que o módulo indecomponível $X$ satisfaz as seguintes condições:

- $\operatorname{Ext}_{A}^{1}(X, X)=0$.

- $\operatorname{Hom}_{A}(\mathcal{F}, X)=0$ е $\operatorname{Hom}_{A}(\mathcal{G}, X)=0$.

- $\operatorname{Ext}_{A}^{1}(\mathcal{F}, X)=0$ e $\operatorname{Ext}_{A}^{1}(\mathcal{G}, X)=0$. 
- $\operatorname{Hom}_{A}\left(\tau^{-t} P_{l}, X\right)=0$ e $\operatorname{Ext}_{A}^{1}\left(\tau^{-t} P_{l}, X\right)=0$.

A primeira observação a se fazer é que todos os módulos $X$ que vamos considerar, exceto $I_{p+q-1}$, são da forma $X \cong \tau^{-k} P_{j}$, com $k \geq 0$, e, portanto, são indecomponíveis e não têm auto-extensões. Além disso, pelo Corolário 2.13 Cap. VIII em [2], não existem morfismos não nulos de um módulo regular a um módulo pós-projetivo, isto é, se $R$ é um módulo regular então $\operatorname{Hom}_{A}\left(R, \tau^{-k} P_{j}\right)=0$.

Por outro lado, pela fórmula de Auslander, temos que

$$
\begin{aligned}
\operatorname{Ext}_{A}^{1}\left(R, \tau^{-k} P_{j}\right) & \cong D \operatorname{Hom}_{A}\left(\tau^{-k} P_{j}, \tau R\right) \\
& \cong D \operatorname{Hom}_{A}\left(P_{j}, \tau^{k+1} R\right) .
\end{aligned}
$$

Portanto, teremos que

$$
\begin{aligned}
\operatorname{Ext}_{A}^{1}\left(\mathcal{F}, \tau^{-k} P_{j}\right)=0 \text { e } \operatorname{Ext}_{A}^{1}\left(\mathcal{G}, \tau^{-k} P_{j}\right)=0 & \Leftrightarrow j \notin \operatorname{Supp} \tau^{k+1} \mathcal{F} \cup \operatorname{Supp} \tau^{k+1} \mathcal{G} \\
& \Leftrightarrow j \in\left(\operatorname{Supp} \tau^{k+1} \mathcal{F}\right)^{\prime} \cap\left(\operatorname{Supp} \tau^{k+1} \mathcal{G}\right)^{\prime}
\end{aligned}
$$

Tendo em vista tais observações, se $\left(F, G, \tau^{-t} P_{l}\right)$ é um sistema estratificante e $X \cong \tau^{-k} P_{j}$, para mostrar que $\left(X \cong \tau^{-k} P_{j}, F, G, \tau^{-t} P_{l}\right)$ é um sistema estratificante é suficiente verificar as seguintes condições:

- $j \in\left(\operatorname{Supp} \tau^{k+1} \mathcal{F}\right)^{\prime} \cap\left(\operatorname{Supp} \tau^{k+1} \mathcal{G}\right)^{\prime}$.

- $\operatorname{Hom}_{A}\left(\tau^{-t} P_{l}, X\right)=0$ e $\operatorname{Ext}_{A}^{1}\left(\tau^{-t} P_{l}, X\right)=0$.

No que segue verificamos estas condições para cada uma das sequências no enunciado da proposição.

1. Vamos ver que $\left(S_{p+q-1}, F, G, P_{0}\right)$ é um sistema estratificante. Como o vértice $p+q-1$ é uma fonte o módulo simples $S_{p+q-1}$ é injetivo e, portanto, claramente

$$
\operatorname{Ext}_{A}^{1}\left(\mathcal{F}, S_{p+q-1}\right)=0, \operatorname{Ext}_{A}^{1}\left(\mathcal{G}, S_{p+q-1}\right)=0 \text { e } \operatorname{Ext}_{A}^{1}\left(P_{0}, S_{p+q-1}\right)=0 .
$$

Por outro lado, usando o Corolário 1.5.5, como $p+q-1 \notin(\operatorname{Supp} \mathcal{F} \cup \operatorname{Supp} \mathcal{G})$ e $S_{p+q-1} \cong$ $I_{p+q-1}$, temos que

$$
\operatorname{Hom}_{A}\left(\mathcal{F}, S_{p+q-1}\right)=0, \operatorname{Hom}_{A}\left(\mathcal{G}, S_{p+q-1}\right)=0 \text { e } \operatorname{Hom}_{A}\left(P_{0}, S_{p+q-1}\right)=0 .
$$

Assim nossa afirmação, que é o caso 1 da lista, está demonstrada.

2. Vamos encontrar agora um $A$-módulo $X$ tal que $\left(X, F, G, P_{p+q-1}\right)$ seja um sistema estratificante. Para isto vamos considerar duas situações: 
- Se $p=q$. Seja $X \cong \tau^{-p+1} P_{0}$. Conforme observamos anteriormente

$$
\left(\operatorname{Supp} \tau^{p} \mathcal{F}\right)^{\prime} \cap\left(\operatorname{Supp} \tau^{p} \mathcal{G}\right)^{\prime}=\{0, p+q-1\}
$$

Além disso, conforme a Proposição $3.2 .10(6), \tau^{-p+1} P_{0}$ não tem $S_{p+q-1}$ como fator de composição. Portanto

$$
\operatorname{Hom}_{A}\left(P_{p+q-1}, \tau^{-p+1} P_{0}\right)=0 .
$$

Assim, neste caso $\tau^{-p+1} P_{0}$ é o módulo que completa nosso sistema estratificante.

- Se $p \neq q$. Consideremos $X=\tau^{-p+1} P_{q-1}$. Desde que

$$
\left(\operatorname{Supp} \tau^{p} \mathcal{F}\right)^{\prime} \cap\left(\operatorname{Supp} \tau^{p} \mathcal{G}\right)^{\prime}=\{q-1\}
$$

e da Proposição 3.2.10 (5), temos que $S_{p+q-1}$ não é fator de composição de $\tau^{-p+1} P_{q-1}$, concluímos que

$$
\operatorname{Hom}_{A}\left(P_{p+q-1}, \tau^{-p+1} P_{q-1}\right)=0 .
$$

Assim, $\left(\tau^{-p+1} P_{q-1}, F, G, P_{p+q-1}\right)$ é um sistema estratificante completo quando $p \neq q$. Em ambos os casos, temos o item 2 da lista.

3. Para completar o sistema estratificante $\left(F, G, \tau^{-t} P_{p+q-1}\right)$ com $t \geq 1$ tal que $p \mid t$ e $q \mid t$ vamos considerar dois casos:

- Se $p=q$. Afirmamos que $\left(\tau^{-t-p+1} P_{0}, F, G, \tau^{-t} P_{p+q-1}\right)$, com $t \geq 1$ tal que $p \mid t$ e $q \mid t$ é um sistema estratificante. De fato,

$$
\begin{aligned}
\left(\operatorname{Supp} \tau^{t+p} \mathcal{F}\right)^{\prime} \cap\left(\operatorname{Supp} \tau^{t+p} \mathcal{G}\right)^{\prime} & =(\operatorname{Supp} \mathcal{F})^{\prime} \cap(\operatorname{Supp} \mathcal{G})^{\prime} \\
& =\{0, p+q-1\} .
\end{aligned}
$$

Por outro lado,

$$
\operatorname{Hom}_{A}\left(\tau^{-t} P_{p+q-1}, \tau^{-t-p+1} P_{0}\right) \cong \operatorname{Hom}_{A}\left(P_{p+q-1}, \tau^{-p+1} P_{0}\right) .
$$

Porém, pela Proposição $3.2 .10(6), S_{p+q-1}$ não é um fator de composição de $\tau^{-p+1} P_{0}$, portanto,

$$
\operatorname{Hom}_{A}\left(\tau^{-t} P_{p+q-1}, \tau^{-t-p+1} P_{0}\right)=0 .
$$

Além disso, pelo Lema 3.2.6 (1), vale que

$$
\operatorname{Ext}_{A}^{1}\left(\tau^{-t} P_{0}, \tau^{-t-p+1} P_{q-1}\right)=0
$$

Tais condições completam a verificação de nossa afirmação. 
- Suponhamos que $p \neq q$. Mostraremos que se $t \geq 1$ e tal que $p \mid t$ e $q \mid t$, então $\left(\tau^{-t-p+1} P_{q-1}, F, G, \tau^{-t} P_{p+q-1}\right)$ é um sistema estratificante completo. De fato,

$$
\begin{aligned}
\left(\operatorname{Supp} \tau^{t+p} \mathcal{F}\right)^{\prime} \cap\left(\operatorname{Supp} \tau^{t+p} \mathcal{G}\right)^{\prime} & =\left(\operatorname{Supp} \tau^{p} \mathcal{F}\right)^{\prime} \cap\left(\operatorname{Supp} \tau^{p} \mathcal{G}\right)^{\prime} \\
& =\{q-1\}
\end{aligned}
$$

e, por outro lado,

$$
\operatorname{Hom}_{A}\left(\tau^{-t} P_{p+q-1}, \tau^{-t-p+1} P_{q-1}\right) \cong \operatorname{Hom}_{A}\left(P_{p+q-1}, \tau^{-p+1} P_{q-1}\right)
$$

Mas da Proposição 3.2.10, (5), como o módulo $\tau^{-p+1} P_{q-1}$ não tem o simples $S_{p+q-1}$ como fator de composição, temos que

$$
\operatorname{Hom}_{A}\left(\tau^{-t} P_{p+q-1}, \tau^{-t-p+1} P_{q-1}\right)=0
$$

Também temos, pelo Lema 3.2.6 parte (1), que

$$
\operatorname{Ext}_{A}^{1}\left(\tau^{-t} P_{p+q-1}, \tau^{-t-p+1} P_{q-1}\right)=0
$$

logo a afirmação está verificada e os casos estudados formam o caso 3 da lista.

4. Mostraremos que $\left(\tau^{-t+1} P_{p+q-1}, F, G, \tau^{-t} P_{0}\right)$, com $t \geq 1$ tal que $p \mid t$ e $q \mid t$ é um sistema estratificante completo. De fato,

$$
\left(\operatorname{Supp} \tau^{t} \mathcal{F}\right)^{\prime} \cap\left(\operatorname{Supp} \tau^{t} \mathcal{G}\right)^{\prime}=(\operatorname{Supp} \mathcal{F})^{\prime} \cap(\operatorname{Supp} \mathcal{G})=\{0, p+q-1\}
$$

Por outro lado,

$$
\begin{aligned}
\operatorname{Hom}_{A}\left(\tau^{-t} P_{0}, \tau^{-t+1} P_{p+q-1}\right) & \cong \operatorname{Hom}_{A}\left(\tau^{-1} P_{0}, P_{p+q-1}\right) \\
& \cong D \operatorname{Ext}_{A}^{1}\left(P_{p+q-1}, \tau^{-1} P_{0}\right) \\
& \cong 0
\end{aligned}
$$

Usando a fórmula de Auslander, obtemos

$$
\begin{aligned}
\operatorname{Ext}_{A}^{1}\left(\tau^{-t} P_{0}, \tau^{-t+1} P_{p+q-1}\right) & \cong D \operatorname{Hom}_{A}\left(\tau^{-t+1} P_{p+q-1}, \tau^{-t+1} P_{0}\right) \\
& \cong D \operatorname{Hom}_{A}\left(P_{p+q-1}, P_{0}\right) \\
& \cong 0
\end{aligned}
$$

pois $P_{0}$ é um projetivo simples. Logo as condições para o sistema estratificante estão verificadas.

5. Provaremos que $\left(\tau^{-t-(p-r-1)} P_{q+r-1}, F, G, \tau^{-t} P_{p-r}\right)$, com $t, r \in \mathbb{N}$ tais que $q \mid t, t \equiv r(\bmod p)$ 
é um sistema estratificante. Pelas hipóteses, temos que

$$
\begin{aligned}
\left(\operatorname{Supp} \tau^{t+(p-r)} \mathcal{F}\right)^{\prime} \cap\left(\operatorname{Supp} \tau^{t+(p-r)} \mathcal{G}\right)^{\prime} & =(\operatorname{Supp} \mathcal{F})^{\prime} \cap\left(\operatorname{Supp} \tau^{p-r} \mathcal{G}\right)^{\prime} \\
& =\{q+r-1\}
\end{aligned}
$$

O Lema 3.2.6, (1), nos garante que $\operatorname{Ext}_{A}^{1}\left(\tau^{-t} P_{p-r}, \tau^{-t-(p-r-1)} P_{q+r-1}\right)=0$.

E finalmente, por termos

$$
\operatorname{Hom}_{A}\left(\tau^{-t} P_{p-r}, \tau^{-t-(p-r-1)} P_{q+r-1}\right) \cong \operatorname{Hom}_{A}\left(P_{p-r}, \tau^{-(p-r-1)} P_{q+r-1}\right)
$$

e segundo a Proposição 3.2.10, (7), por $S_{p-r}$ não ser fator de composição de $\tau^{-(p-r-1)} P_{q+r-1}$, resulta que

$$
\operatorname{Hom}_{A}\left(\tau^{-t} P_{p-r}, \tau^{-t-(p-r-1)} P_{q+r-1}\right)=0
$$

6. Suponhamos que $t, r \in \mathbb{N}$ são tais que $p \mid t, t \equiv r(\bmod q)$ e $1 \leq r \leq q-1$. Vamos considerar dois casos:

- Suponhamos que $p \leq q-r$. Então, pelas hipóteses sobre $t$, temos que

$$
\begin{aligned}
\left(\operatorname{Supp} \tau^{t+q-r} \mathcal{F}\right)^{\prime} \cap\left(\operatorname{Supp} \tau^{t+q-r} \mathcal{G}\right)^{\prime} & =\left(\operatorname{Supp} \tau^{q-r} \mathcal{F}\right)^{\prime} \cap(\operatorname{Supp} \mathcal{G})^{\prime} \\
& =\{p-q+r\}
\end{aligned}
$$

Por outro lado, como $q-r-1 \geq 0$, pelo Lema 3.2.6, (1), garante que

$$
\operatorname{Ext}_{A}^{1}\left(\tau^{-t} P_{p+q-r-1}, \tau^{-t-q+r+1} P_{p-q+r}\right)=0
$$

Além disso, de

$$
\operatorname{Hom}_{A}\left(\tau^{-t} P_{p+q-r-1}, \tau^{-t-q+r+1} P_{p-q+r}\right) \cong \operatorname{Hom}_{A}\left(P_{p+q-r-1}, \tau^{-q+r+1} P_{p-q+r}\right)
$$

e da Proposição 3.2.10, (4), em que $S_{p+q-r-1}$ não é fator de composição de $\tau^{-q+r+1} P_{p-q+r}$, resulta que

$$
\operatorname{Hom}_{A}\left(\tau^{-t} P_{p+q-r-1}, \tau^{-t-q+r+1} P_{p-q+r}\right)=0 .
$$

Dessa forma a sequência $\left(\tau^{-t-q+r+1} P_{p-q+r}, F, G, \tau^{-t} P_{p+q-r-1}\right)$, com $t \geq 1$ e $r$ tais que $p \mid t, t \equiv r(\bmod q), 1 \leq r \leq q-1$ e $q-r \geq p$ é um sistema estratificante completo.

- Suponhamos agora que $q-r>p$ e seja $l$ tal que $p+l=q-r$. Nessas condições,

$$
\begin{aligned}
\left(\operatorname{Supp} \tau^{t+p-1} \mathcal{F}\right)^{\prime} \cap\left(\operatorname{Supp} \tau^{t+p-1} \mathcal{G}\right)^{\prime} & =\left(\operatorname{Supp} \tau^{-l} \mathcal{F}\right)^{\prime} \cap(\operatorname{Supp} \mathcal{G})^{\prime} \\
& =\{p+l-1\} \\
& =\{q-r-1\}
\end{aligned}
$$


Agora, pelo Lema 3.2.6, (1), temos que

$$
\operatorname{Ext}_{A}^{1}\left(\tau^{-t} P_{p+q-r-1}, \tau^{-t-p-1} P_{q-r-1}\right)=0 .
$$

O fato de que

$$
\operatorname{Hom}_{A}\left(\tau^{-t} P_{p+q-r-1}, \tau^{-t-p+1} P_{q-r-1}\right) \cong \operatorname{Hom}_{A}\left(P_{p+q-r-1}, \tau^{-p+1} P_{q-r-1}\right) .
$$

e da Proposição $3.2 .10,(4)$, que garante que $S_{p+q-r-1}$ não é fator de composição de $\left.\tau^{-p+1} P_{q-r-1}\right)$, temos que $\operatorname{Hom}_{A}\left(\tau^{-t} P_{p+q-r-1}, \tau^{-t-p+1} P_{q-r-1}\right)=0$.

$\operatorname{Assim}\left(\tau^{-t-p+1} P_{q-r-1}, F, G, \tau^{-t} P_{p+q-r-1}\right) \operatorname{com} t \geq 1$, tal que $p \mid t, t \equiv r(\bmod q)$, com $1 \leq r \leq q-1$ e $q-r>p$ é um sistema estratificante completo.

Usando o carcás $\Gamma\left(\bmod \triangle\left(\widetilde{\mathbb{A}}_{p, q}\right)\right)$ e o Lema 3.2.9, é possível obter informações sobre os fatores de composição dos $A$-módulos pré-injetivos. Reunimos tais informações na seguinte proposição, cuja prova é análoga à prova da Proposição 3.2.10.

Proposição 3.2.12. Seja $A$ é a $K$-álgebra $K \triangle\left(\widetilde{\mathbb{A}}_{p, q}\right)$. Então a componente pré-injetiva de $\Gamma(\bmod A)$ satisfaz as condições abaixo:

1. Todos os morfismos irredutiveis na componente pré-injetiva do carcás $\Gamma\left(\bmod \triangle\left(\widetilde{\mathbb{A}}_{p, q}\right)\right)$ são epimorfismos.

2. O módulo $I_{0}$ e todos seus antecessores são módulos sinceros.

3. O inteiro $r$ minimal com a propriedade de que todos os módulos da forma $\tau^{r} I_{i}$, com $i \in$ $\{0, \ldots, p+q-1\}$, são sinceros é $r=p$.

4. Se $1 \leq i \leq p-1$, então:

- o menor inteiro $r$ tal que $\tau^{r} I_{i}$ é um módulo sincero é $r=i$. Ademais, todos os $A$ módulos da forma $\tau^{k} I_{i}$, com $k \geq i$, são A-módulos sinceros.

- se $k<i$ então os fatores de composição de $\tau^{k} I_{i}$ são os A-módulos simples $S_{j}$ com $i-k \leq j \leq p-1$ e $p+q-k \leq j \leq p+q-1$.

5. Se $p \leq i<p+q-1$, então:

- se $p \leq i<q-1$, o menor inteiro $r$ tal que $\tau^{r} I_{i}$ é um módulo sincero é $r=i-p+1$. Ademais, todo módulo da forma $\tau^{r} I_{i}$ com $r \geq i-p+1$ é um módulo sincero.

- se $q-1 \leq i \leq p+q-1$, então o menor inteiro $r$ tal que $\tau^{r} I_{i}$ é sincero é $r=p$. Ademais, todo módulo da forma $\tau^{r} I_{i}$, com $r \geq p$ é sincero. 
- Se $p \leq i \leq p+q-2$ e $k$ é tal que $\tau^{k} I_{i}$ não é um módulo sincero, então os fatores de composição de $\tau^{k} I_{i}$ são os A-módulos simples $S_{j}$ com $i-k \leq j \leq p+q-1$ e $p-k \leq j \leq p-1$.

6. Se $k<p$, então os fatores de composição de $\tau^{k} I_{p+q-1}$ são os simples $S_{j}$ com $p-k \leq j \leq$ $p-1$ e $p+q-k \leq j \leq p+q-1$.

Usando a Proposição 3.2.12 e a Proposição 3.2.8, que caracteriza os sistemas estratificantes da forma $(F, G, Y)$, onde $Y$ é um $A$-módulo pré-injetivo, podemos obter os sistemas estratificantes completos sobre $A$ do tipo $(X, F, G, Y)$.

Notemos que, uma vez que o Lema 2.3.17 garante que o módulo $X$ que completa o sistema estratificante $(F, G, Y)$ é único, quando encontrado um módulo $X$ satisfazendo todas as condições necessárias a busca está terminada. Assim, o trabalho consistiu em procurar todos os tais módulos, que resultaram ser todos módulos pré-injetivos, e a prova consistirá de verificar que os módulos encontrados completam o sistema estratificante.

Proposição 3.2.13. Seja $A=K Q$, onde $Q=\triangle\left(\widetilde{\mathbb{A}}_{p, q}\right)$. Então a lista de todos os sistemas estratificantes da forma $(X, F, G, Y)$, onde $Y$ é um A-módulo pré-injetivo, é a seguinte:

1. $\left(\tau^{t} I_{p-1}, F, G, \tau^{t} I_{p}\right)$, com $t \geq 1$ tal que $t \equiv p-1(\bmod p) e q \mid t$.

2. $\left(\tau^{t} I_{p+q-2}, F, G, \tau^{t} I_{1}\right)$, com $t \geq 1$ tal que $t \equiv q-1(\bmod q)$ e $p \mid t$.

3. $\left(\tau^{t+1} I_{p+q-1}, F, G, \tau^{t} I_{0}\right)$, com $t \geq 1$ tal que $t \equiv p-1(\bmod p) e$ $t \equiv q-1(\bmod q)$.

4. $\left(\tau^{t-p+1} I_{q-1}, F, G, \tau^{t} I_{p+q-1}\right)$, com $t \geq 1$ tal que $t \equiv p-1(\bmod p)$ e $t \equiv q-1(\bmod q)$.

5. $\left(\tau^{t-r} I_{p+q-r-2}, F, G, \tau^{t} I_{r+1}\right)$, com $t \geq 1$ tal que $t \equiv r(\bmod p), 0<r<p-1$ e $t \equiv$ $q-1(\bmod q)$.

6. $\left(X, F, G, \tau^{t} I_{p+r}\right)$, com $t \geq 1$ tal que $t \equiv r(\bmod q), 0<r<q-1$ e $t \equiv p-1(\bmod p)$, onde

$$
X \cong\left\{\begin{array}{l}
\tau^{t-r} I_{p-(r+1)}, \text { se } r<p \\
\tau^{t-(p-1)} I_{r}, \text { se } r \geq p
\end{array}\right.
$$

Demonstração. Os Lemas 2.3.16 e 2.3.17 garantem que existe um único módulo $X$ tal que $\left(X, F, G, \tau^{m} I_{i}\right)$ é um sistema estratificante. Portanto a demonstração consistirá em verificar que o módulo indecomponível $X$ satisfaz as seguintes condições:

- $\operatorname{Ext}_{A}^{1}(X, X)=0$.

- $\operatorname{Hom}_{A}(\mathcal{F}, X)=0$ e $\operatorname{Hom}_{A}(\mathcal{G}, X)=0$. 
- $\operatorname{Ext}_{A}^{1}(\mathcal{F}, X)=0$ e $\operatorname{Ext}_{A}^{1}(\mathcal{G}, X)=0$.

- $\operatorname{Hom}_{A}\left(\tau^{t} I_{i}, X\right)=0$ e $\operatorname{Ext}_{A}^{1}\left(\tau^{t} I_{i}, X\right)=0$.

Todos os módulos $X$ que vamos considerar são da forma $X \cong \tau^{l} I_{j}$ e, portanto, são indecomponíveis e não têm auto-extensões. Além disso, notemos que se $X$ é um $A$-módulo pré-injetivo e $R$ é um $A$-módulo regular então, usando a fórmula de Auslander temos que $\operatorname{Ext}_{A}^{1}(R, X) \cong$ $D \operatorname{Hom}_{A}(X, \tau R)$. Mas, segundo o Corolário 2.13 (VIII) em [2], não existem morfismos não nulos de um módulo pré-injetivo a um módulo regular, portanto

$$
\operatorname{Ext}_{A}^{1}(R, X) \cong D \operatorname{Hom}_{A}(X, \tau R)=0 .
$$

Por outro lado, para $l \geq 0$, temos que $\operatorname{Hom}_{A}\left(R, \tau^{l} I_{j}\right) \cong \operatorname{Hom}_{A}\left(\tau^{-l} R, I_{j}\right)$. Segundo o Corolário 1.5.5, segue que

$$
\operatorname{Hom}_{A}\left(R, \tau^{l} I_{j}\right)=0 \Leftrightarrow j \notin \operatorname{supp} \tau^{-l} R
$$

Portanto, teremos que

$$
\operatorname{Hom}_{A}\left(\mathcal{F}, \tau^{l} I_{j}\right)=0 \text { e } \operatorname{Hom}_{A}\left(\mathcal{G}, \tau^{l} I_{j}\right)=0 \Leftrightarrow j \in\left(\operatorname{Supp} \tau^{-l} \mathcal{F}\right)^{\prime} \cap\left(\operatorname{Supp} \tau^{-l} \mathcal{G}\right)^{\prime} .
$$

Tendo em vista as anteriores observações, se $\left(F, G, \tau^{t} I_{i}\right)$ é um sistema estratificante e $X \cong$ $\tau^{l} I_{j}$, para mostrar que $\left(X, F, G, \tau^{t} I_{i}\right)$ é um sistema estratificante é suficiente verificar que:
a. $\quad j \in\left(\operatorname{Supp} \tau^{-l} \mathcal{F}\right)^{\prime} \cap\left(\operatorname{Supp} \tau^{-l} \mathcal{G}\right)^{\prime}$
b. $\operatorname{Hom}_{A}\left(\tau^{t} I_{i}, X\right)=0$ e $\operatorname{Ext}_{A}^{1}\left(\tau^{t} I_{i}, X\right)=0$.

Consideraremos aqui os vários casos, que dependem da forma de $t$, de acordo com a lista no enunciado da Proposição 3.2.8.

1. Seja $t \geq 1$ tal que $t \equiv p-1(\bmod p)$ e $q \mid t$. Então, pelo Corolário 3.2.4, (1b), seguem as igualdades

$$
\left(\operatorname{Supp} \tau^{-t} \mathcal{F}\right)^{\prime} \cap\left(\operatorname{Supp} \tau^{-t} \mathcal{G}\right)^{\prime}=\left(\operatorname{Supp} \tau^{-(p-1)} \mathcal{F}\right)^{\prime} \cap\left(\operatorname{Supp} \tau^{-t} \mathcal{G}\right)^{\prime}=\{p-1\} .
$$

Observemos que $\operatorname{Hom}_{A}\left(\tau^{t} I_{p}, \tau^{t} I_{p-1}\right) \cong \operatorname{Hom}_{A}\left(I_{p}, I_{p-1}\right)$ e que $I_{p}$ tem a seguinte representação

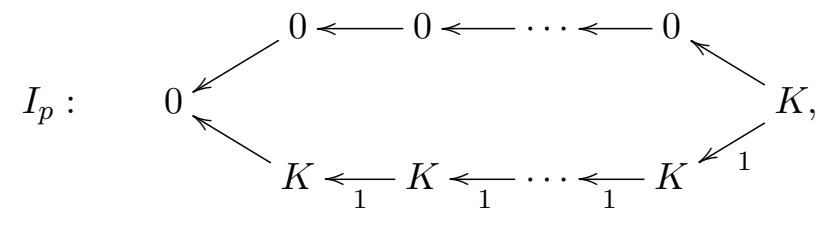

o que mostra que $S_{p-1}$ não é fator de composição $I_{p}$. Portanto, $\operatorname{Hom}_{A}\left(\tau^{t} I_{p}, \tau^{t} I_{p-1}\right)=0$.

Além disso, o Lema 3.2.6, (2), estabelece que $\operatorname{Ext}_{A}^{1}\left(\tau^{t} I_{p}, \tau^{t} I_{p-1}\right)=0$. 
Assim tomando $X \cong \tau^{t} I_{p-1}$, temos pelas condições (a) e (b) que $\left(\tau^{t} I_{p-1}, F, G, \tau^{t} I_{p}\right)$, com $t \geq 1$ tal que $t \equiv p-1(\bmod p)$ e $q \mid t$, é um sistema estratificante completo sobre $A$.

2. Sejam $t \geq 1$ tal que $t \equiv q-1(\bmod q)$ e $p \mid t$ e $X \cong \tau^{t} I_{p+q-2}$. Pelo Corolário 3.2.4, (2b), temos que

$$
\left(\operatorname{Supp} \tau^{-t} \mathcal{F}\right)^{\prime} \cap\left(\operatorname{Supp} \tau^{-t} \mathcal{G}\right)^{\prime}=\left(\operatorname{Supp} \tau^{-t} \mathcal{F}\right)^{\prime} \cap\left(\operatorname{Supp} \tau^{-(q-1)} \mathcal{G}\right)^{\prime}=\{p+q-2\}
$$

Por outro lado, $\operatorname{Hom}_{A}\left(\tau^{t} I_{1}, \tau^{t} I_{p+q-2}\right) \cong \operatorname{Hom}_{A}\left(I_{1}, I_{p+q-2}\right)$ e como $I_{1}$ é o módulo associado à representação

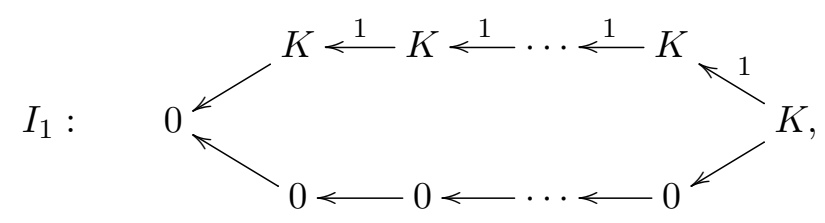

que não tem $S_{p+q-2}$ como fator de composição, então $\operatorname{Hom}_{A}\left(\tau^{t} I_{1}, \tau^{t} I_{p+q-2}\right)=0$.

O Lema 3.2.6, (2), garante que $\operatorname{Ext}_{A}^{1}\left(\tau^{t} I_{1}, \tau^{t} I_{p+q-2}\right)=0$.

Assim $\left(\tau^{t} I_{p+q-2}, F, G, \tau^{t} I_{1}\right)$, com $t \geq 1$ tal que $t \equiv q-1(\bmod q)$ e $p \mid t$, é um sistema estratificante completo sobre $A$.

3. Sejam $t \geq 1$ tal que $t \equiv p-1(\bmod p)$ e $t \equiv q-1(\bmod q)$ e $X \cong \tau^{t+1} I_{p+q-1}$. Pelo Corolário 3.2.4, temos que

$$
\left(\operatorname{Supp} \tau^{-t-1} \mathcal{F}\right)^{\prime} \cap\left(\operatorname{Supp} \tau^{-t-1} \mathcal{G}\right)^{\prime}=(\operatorname{Supp} \mathcal{F})^{\prime} \cap(\operatorname{Supp} \mathcal{G})^{\prime}=\{0, p+q-1\}
$$

o que mostra que a condição (a) está verificada. Por outro lado, pela fórmula de Auslander, temos que

$$
\operatorname{Hom}_{A}\left(\tau^{t} I_{0}, \tau^{t+1} I_{p+q-1}\right) \cong \operatorname{Hom}_{A}\left(I_{0}, \tau I_{p+q-1}\right) \cong D \operatorname{Ext}_{A}^{1}\left(I_{p+q-1}, I_{0}\right)=0
$$

A fórmula de Auslander também mostra que

$$
\begin{aligned}
\operatorname{Ext}_{A}^{1}\left(\tau^{t} I_{0}, \tau^{t+1} I_{p+q-1}\right) & \cong D \operatorname{Hom}_{A}\left(\tau^{t+1} I_{p+q-1}, \tau^{t+1} I_{0}\right) \\
& \cong \operatorname{Hom}_{A}\left(I_{p+q-1}, I_{0}\right) \\
& =0
\end{aligned}
$$

pois $I_{p+q-1}$ é um injetivo simples, completando a condição (b).

Assim $\left(\tau^{t+1} I_{p+q-1}, F, G, \tau^{t} I_{0}\right)$, com $t \geq 1$ tal que $t \equiv q-1(\bmod q)$ e $t \equiv p-1(\bmod p)$ é um sistema estratificante completo sobre $A$.

4. Sejam $t \geq 1$ tal que $t \equiv p-1(\bmod p)$ e $t \equiv q-1(\bmod q)$. Vamos mostrar que a sequência $\left(\tau^{t-p+1} I_{q-1}, F, G, \tau^{t} I_{p+q-1}\right)$ é um sistema estratificante completo sobre A. Pelo Corolário 
3.2 .4 , temos que

$$
\begin{aligned}
\left(\operatorname{Supp} \tau^{-t+p-1} \mathcal{F}\right)^{\prime} \cap\left(\operatorname{Supp} \tau^{-t+p-1} \mathcal{G}\right)^{\prime} & =(\operatorname{Supp} \mathcal{F})^{\prime} \cap\left(\operatorname{Supp} \tau^{-(q-p)} \mathcal{G}\right)^{\prime} \\
& =\{q-1\}
\end{aligned}
$$

comprovando (a). Por outro lado

$$
\operatorname{Hom}_{A}\left(\tau^{t} I_{p+q-1}, \tau^{t-(p-1)} I_{q-1}\right) \cong \operatorname{Hom}_{A}\left(\tau^{p-1} I_{p+q-1}, I_{q-1}\right)=0,
$$

pois, pela Proposição 3.2.12, (5), $S_{q-1}$ não é um fator de composição de $\tau^{p-1} I_{p+q-1}$.

Além disso, pelo Lema 3.2.6, (2), vale que

$$
\operatorname{Ext}_{A}^{1}\left(\tau^{t} I_{p+q-1}, \tau^{t-(p-1)} I_{q-1}\right)=0
$$

e a condição (b) está verificada. Resultando que $\left(\tau^{t-p+1} I_{q-1}, F, G, \tau^{t} I_{p+q-1}\right)$, com $t \geq 1$ tal que $t \equiv p-1(\bmod p)$ e $t \equiv q-1(\bmod q)$, é um sistema estratificante completo sobre $A$.

5. Consideremos $t \geq 1$ tal que $t \equiv r(\bmod p), 0<r<p-1$ e $t \equiv q-1(\bmod q)$. Seja $X \cong \tau^{t-r} I_{p+q-r-2}$. Para a condição (a), usando o Corolário 3.2.4, temos que

$$
\begin{aligned}
\left(\operatorname{Supp} \tau^{-(t-r)} \mathcal{F}\right)^{\prime} \cap\left(\operatorname{Supp} \tau^{-(t-r)} \mathcal{G}\right)^{\prime} & =(\operatorname{Supp} \mathcal{F})^{\prime} \cap\left(\operatorname{Supp} \tau^{-(t-r)} \mathcal{G}\right)^{\prime} \\
& =\left(\operatorname{Supp} \tau^{-(q-1-r)} \mathcal{G}\right)^{\prime} \\
& =\{p+q-r-2\}
\end{aligned}
$$

Para a condição (b), temos primeiramente

$$
\operatorname{Hom}_{A}\left(\tau^{t} I_{r+1}, \tau^{t-r} I_{p+q-r-2}\right) \cong \operatorname{Hom}_{A}\left(\tau^{r} I_{r+1}, I_{p+q-r-2}\right)=0,
$$

uma vez que pela Proposição 3.2 .12 parte $(5), \tau^{r} I_{r+1}$ não tem $S_{p+q-r-2}$ como fator de composição.

Temos também do Lema 3.2.6, (2), que $\operatorname{Ext}_{A}^{1}\left(\tau^{t} I_{r+1}, \tau^{t-r} I_{p+q-r-2}\right)=0$. Logo de (a) e (b) estarem verificadas, temos que $\left(\tau^{t-r} I_{p+q-r-2}, F, G, \tau^{t} I_{r+1}\right)$ é um sistema estratificante completo sobre $A$.

6. Seja $t \geq 1$ tal que $t \equiv r(\bmod q), 0<r<q-1$ e $t \equiv p-1(\bmod p)$. Para completar o sitema estratificante $\left(F, G, \tau^{t} I_{p+r}\right)$ vamos considerar duas situações:

- Suponhamos que $r<p$. Seja $X \cong \tau^{t-r} I_{p-(r+1)}$. Temos que

$$
\begin{aligned}
& \left.\operatorname{Supp} \tau^{-(t-r)} \mathcal{F}\right)^{\prime} \cap\left(\operatorname{Supp} \tau^{-(t-r)} \mathcal{G}\right)^{\prime}=\left(\operatorname{Supp} \tau^{-(p-1-r)} \mathcal{F}\right)^{\prime} \cap(\operatorname{Supp} \mathcal{G})^{\prime} \\
& =\left(\operatorname{Supp} \tau^{(r+1)} \mathcal{F}\right)^{\prime} \\
& =\{p-(r+1)\} \text {, }
\end{aligned}
$$


com o que a condição (a) está verificada.

Também temos, neste caso, que

$$
\operatorname{Hom}_{A}\left(\tau^{t} I_{p+r}, \tau^{t-r} I_{p-(r+1)}\right) \cong \operatorname{Hom}_{A}\left(\tau^{r} I_{p+r}, I_{p-(r+1)}\right)=0
$$

pois, pela Proposição 3.2.12, $(5), \tau^{r} I_{p+r}$ não tem $S_{p-(r+1)}$ como fator de composição. O Lema 3.2.6, (2), garante por sua vez que

$$
\operatorname{Ext}_{A}^{1}\left(\tau^{t} I_{p+r}, \tau^{t-r} I_{p-(r+1)}\right)=0
$$

e então a condição (b) também está verificada.

Asim temos que $\left(\tau^{t-r} I_{p-(r+1)}, F, G, \tau^{t} I_{p+r}\right) \operatorname{com} t \equiv r(\bmod q), 0<r<q-1$, $t \equiv p-1(\bmod p)$ e $r<p$ é um sistema estratificante completo.

- Suponhamos que $r \geq p$ e seja $X \cong \tau^{t-(p-1)} I_{r}$. Sob as hipóteses de $t, r$ e $p$, temos que

$$
\begin{aligned}
\left.\operatorname{Supp} \tau^{-[t-(p-1)]} \mathcal{F}\right)^{\prime} \cap\left(\operatorname{Supp} \tau^{-[t-(p-1)]} \mathcal{G}\right)^{\prime} & =\left(\operatorname{Supp} \tau^{-[r-(p-1)]} \mathcal{G}\right)^{\prime} \\
& =\{p+(r-p+1)-1\} \\
& =\{r\},(\operatorname{condição~(a)).~}
\end{aligned}
$$

Do isomorfismo $\operatorname{Hom}_{A}\left(\tau^{t} I_{p+r}, \tau^{t-(p-1)} I_{r}\right) \cong \operatorname{Hom}_{A}\left(\tau^{p-1} I_{p+r}, I_{r}\right)$ e da Proposição 3.2 .12 , (5), por $S_{r}$ não ser fator de composição de $\tau^{r} I_{p+r}$ temos que

$$
\operatorname{Hom}_{A}\left(\tau^{t} I_{p+r}, \tau^{t-(p-1)} I_{r}\right)=0
$$

Também o Lema 3.2.6, (2.b), diz que

$$
\operatorname{Ext}_{A}^{1}\left(\tau^{t} I_{p+r}, \tau^{t-(p-1)} I_{r}\right)=0
$$

garantindo a validade de (b). Ou seja, $\left(\tau^{t-(p-1)} I_{r}, F, G, \tau^{t} I_{p+r}\right)$ com $t \equiv r(\bmod q)$, $0<r<q-1, t \equiv p-1(\bmod p)$ e $r \geq p$ é um sistema estratificante completo sobre $A$, completando a lista no enunciado da proposição.

As Proposições 3.2.11 e 3.2.13 fornecem una lista completa dos sistemas estratificantes completos da forma $(X, F, G, Y)$ sobre $A=K Q$, onde $Q=\triangle\left(\widetilde{\mathbb{A}}_{p, q}\right)$. Apresentamos tal lista no seguinte teorema.

Teorema 3.2.14. Sejam $A=K \triangle\left(\widetilde{\mathbb{A}}_{p, q}\right)$ e $F$ e $G$ sistemas estratificantes como definidos em 3.5 e 3.6. A lista completa dos sistemas estratificantes da forma $(X, F, G, Y)$ é a seguinte:

1. $\left(S_{p+q-1}, F, G, P_{0}\right)$. 
2. $\left(X, F, G, P_{p+q-1}\right)$, onde

$$
X \cong\left\{\begin{array}{l}
\tau^{-p+1} P_{0}, \text { se } p=q \\
\tau^{-p+1} P_{q-1}, \text { se } p \neq q
\end{array}\right.
$$

3. $\left(X, F, G, \tau^{-t} P_{p+q-1}\right)$ com $t \geq 1$ tal que $p \mid t$ e $q \mid t$, onde

$$
X \cong\left\{\begin{array}{l}
\tau^{-t-p+1} P_{0}, \text { se } p=q \\
\tau^{-t-p+1} P_{q-1}, \text { se } p \neq q
\end{array}\right.
$$

4. $\left(\tau^{-t+1} P_{p+q-1}, F, G, \tau^{-t} P_{0}\right)$, com $t \geq 1$ tal que $p \mid t$ e $q \mid t$.

5. $\left(\tau^{-t-(p-r-1)} P_{q+r-1}, F, G, \tau^{-t} P_{p-r}\right)$, com $t \geq 1$ tal que $q \mid t$ e $r$ tal que $t \equiv r(\bmod p) e$ $1 \leq r \leq p-1$.

6. $\left(X, F, G, \tau^{-t} P_{p+q-r-1}\right)$, com $t \geq 1$ tal que $p \mid t$ e $r$ tal que $t \equiv r(\bmod q)$ e $1 \leq r \leq q-1$.

$$
X \cong\left\{\begin{array}{l}
\tau^{-t-q+r+1} P_{p-q+r}, \text { se } p \leq q-r \\
\tau^{-t-p+1} P_{q-r-1}, \text { se } p>q-r
\end{array}\right.
$$

7. $\left(\tau^{t} I_{p-1}, F, G, \tau^{t} I_{p}\right)$, com $t \geq 1$ tal que $t \equiv p-1(\bmod p)$ e $q \mid t$.

8. $\left(\tau^{t} I_{p+q-2}, F, G, \tau^{t} I_{1}\right)$, com $t \geq 1$ tal que $t \equiv q-1(\bmod q)$ e $p \mid t$.

9. $\left(\tau^{t+1} I_{p+q-1}, F, G, \tau^{t} I_{0}\right)$, com $t \geq 1$ tal que $t \equiv p-1(\bmod p)$ e $t \equiv q-1(\bmod q)$.

10. $\left(\tau^{t-p+1} I_{q-1}, F, G, \tau^{t} I_{p+q-1}\right)$, com $t \geq 1$ tal que $t \equiv p-1(\bmod p)$ e $t \equiv q-1(\bmod q)$.

11. $\left(\tau^{t-r} I_{p+q-r-2}, F, G, \tau^{t} I_{r+1}\right)$, com $t \geq 1$ tal que $t \equiv r(\bmod p), 0<r<p-1$ et $t \equiv$ $q-1(\bmod q)$.

12. $\left(X, F, G, \tau^{t} I_{p+r}\right)$, com $t \geq 1$ tal que $t \equiv r(\bmod q), 0<r<q-1 e t \equiv p-1(\bmod p)$, onde

$$
X \cong\left\{\begin{array}{l}
\tau^{t-r} I_{p-(r+1)}, \text { se } r<p \\
\tau^{t-(p-1)} I_{r}, \text { se } r \geq p
\end{array}\right.
$$




\section{Capítulo 4}

\section{Sistemas estratificantes via módulos inclinantes em álgebras hereditárias}

Sejam $A$ uma álgebra hereditária, $T$ é um $A$-módulo inclinante parcial básico e $\oplus_{i=1}^{n} T_{i}$ sua decomposição em somandos diretos indecomponíveis. Surge a seguinte pergunta, se $\left(T_{1}, \ldots, T_{n}\right)$ é sequência dos somandos de $T$, será que ela é uma sequência especial?

Embora não tenha sido possível responder plenamente a questão, neste capítulo estabelecemos que dado um $A$-módulo inclinante parcial básico $T$ sempre existe uma sequência especial dos seus somandos diretos. Depois, encontramos condições, suficientes e necessárias para que uma sequência da formma $\left(T_{1}, T_{2}\right)$, onde $T=T_{1} \oplus T_{2}$ é um módulo incliante parcial básico, seja uma sequência especial. Finalmente, tratamos o problema análogo para uma sequência de três módulos.

Começaremos enunciando resultados da teoria de inclinação que usaremos como ferramenta ao longo deste capítulo.

Lema 4.1.1. (3.2 Cap. VI em [2]) Sejam A uma K-álgebra, T um A-módulo inclinante e $B=\operatorname{End}_{A}(T) . S e M, N \in \mathcal{T}(T)=\left\{X \in \bmod A / \operatorname{Ext}_{A}^{1}(T, X)=0\right\}$, então existem isomorfismos funtoriais:

1. $\left.\operatorname{Hom}_{A}(M, N) \cong \operatorname{Hom}_{B}\left(\operatorname{Hom}_{A}(T, M)\right), \operatorname{Hom}_{A}(T, N)\right)$.

2. $\left.\operatorname{Ext}_{A}^{1}(M, N) \cong \operatorname{Ext}_{B}^{1}\left(\operatorname{Hom}_{A}(T, M)\right), \operatorname{Hom}_{A}(T, N)\right)$.

Lema 4.1.2. (3.10 Cap. VI em [2]) Sejam $A$ uma $K$-álgebra, $T_{A}$ um A-módulo inclinante básico, $T_{A}=T_{1} \oplus \ldots \oplus T_{n}$ sua decomposição em somandos diretos indecomponíveis e dois a dois não isomorfos, e $B=\operatorname{End}_{A}(T)$. Seja $e_{i} \in B=\operatorname{End}_{A}(T)$ a composição da projeção canônica $p_{i}: T \longrightarrow T_{i}$ com a inclusão canônica $u_{i}: T_{i} \longrightarrow T$. Então o conjunto $\left\{e_{1}, \ldots, e_{n}\right\}$ é um conjunto 
completo de idempotentes ortogonais e primitivos de $B$ e existe um isomorfismo de B-módulos

$$
e_{a} B \cong \operatorname{Hom}_{A}\left(T, T_{a}\right), \text { para todo } a \in\{1, \ldots n\}
$$

Lema 4.1.3. (3.4 Cap. VIII em [2]) Sejam $A=K Q$, onde $Q$ é um carcás finito, conexo e acíclico e $T$ um $A$-módulo inclinante. Se $B=\operatorname{End}_{A}(T)$, então o carcás ordinário $Q_{B}$ de $B$ é acíclico.

Se $A$ é uma álgebra hereditária, dado um conjunto completo de idempotentes ortogonais e primitivos $\left\{e_{a_{1}}, \ldots, e_{a_{n}}\right\}$ de $A$ existe uma enumeração dos seus elementos de tal forma que o módulo estândar $\triangle_{i}$ coincide com o projetivo $P_{i}$, para todo $i=1, \ldots, n$. Tal enumeração é conhecida como enumeração admissível, cuja definição é a seguinte.

Definição 4.1.4. Seja $Q=\left(Q_{0}, Q_{1}\right)$ um carcás tal que $\left|Q_{0}\right|=n$. Uma enumeração admissível de $Q$ é uma bijeção entre $Q_{0}$ e o conjunto $\{1,2, \ldots n\}$ tal que se existir uma flecha $v_{i} \longrightarrow v_{j}$, então $i>j$.

Na seguinte observação veremos que, se $Q$ é um carcás finito e acíclico sempre é possível construir uma enumeração admissível para $Q$.

Observação 4.1.5. Seja $Q=\left(Q_{0}, Q_{1}\right)$ um carcás finito, conexo e acíclico tal que $\left|Q_{0}\right|=n$. Então podemos construir uma enumeração admissível de $Q$ da seguinte forma: seja $v_{1}$ um poço em $Q$. Então consideramos o sub-carcás pleno $Q(1)$ de $Q$ tal que seu conjunto de vértices é $Q_{0}-\left\{v_{1}\right\}$. Seja $v_{2}$ um poço em $Q(1)$. Continuamos indutivamente.

Proposição 4.1.6. Sejam A uma álgebra hereditária e T um A-módulo inclinante básico. Então existe uma sequência especial de $T$.

Demonstração. Seja $B=\operatorname{End}_{A}(T)$. Então, pelo Lema 4.1.3, o carcás ordinário $Q_{B}$ de $B$ é acíclico e então, pela observação acima, existe uma enumeração admissível de $Q_{B}$. Seja $\left\{e_{1}, \ldots, e_{n}\right\}$ um conjunto completo de idempotentes ortogonais e primitivos de $B$ enumerados de tal forma que os vértices correspondentes estão enumerados de forma admissível. Seja $\boldsymbol{e}=\left(e_{1}, \ldots, e_{n}\right)$. Notemos que, com a ordem $e$, se $j>i$ então não existem morfismos não nulos do projetivo $P_{j}$ para o projetivo $P_{i}$ e, portanto, $\triangle_{i}=P_{i}$, para $i=1, \ldots, n$. Por outro lado, pelo Lema 4.1.2, temos que $P_{a} \cong e_{a} B \cong \operatorname{Hom}_{A}\left(T, T_{a}\right)$. Assim, na ordem e a sequência $\left(\operatorname{Hom}_{A}\left(T, T_{1}\right), \ldots, \operatorname{Hom}_{A}\left(T, T_{n}\right)\right)$ é a sequência de $B$-módulos estândares à esquerda. Portanto, temos que

$$
\begin{gathered}
\operatorname{Hom}_{B}\left(\operatorname{Hom}_{A}\left(T, T_{i}\right), \operatorname{Hom}_{A}\left(T, T_{j}\right)\right)=0, \text { se } i>j, \text { e } \\
\operatorname{Ext}_{B}^{1}\left(\operatorname{Hom}_{A}\left(T, T_{i}\right), \operatorname{Hom}_{A}\left(T, T_{j}\right)\right)=0, \text { se } i \geq j
\end{gathered}
$$


Mas, por outro lado, pelo Lema 4.1.1, vale que

$$
\operatorname{Hom}_{B}\left(\operatorname{Hom}_{A}\left(T, T_{i}\right), \operatorname{Hom}_{A}\left(T, T_{j}\right)\right) \cong \operatorname{Hom}_{A}\left(T_{i}, T_{j}\right)
$$

e que

$$
\operatorname{Ext}_{B}^{1}\left(\operatorname{Hom}_{A}\left(T, T_{i}\right), \operatorname{Hom}_{A}\left(T, T_{j}\right)\right) \cong \operatorname{Ext}_{A}^{1}\left(T_{i}, T_{j}\right) .
$$

Concluímos que $\left(T_{1}, \ldots, T_{n}\right)$ é um sistema estratificante sobre $A$ e, portanto, que existe uma sequência especial de $T$.

Observemos que, se $T$ é um módulo inclinante parcial básico então existe uma sequência especial de $T$. Para constuir tal sequência primeiro completamos o módulo $T$ a um módulo inclinante $T^{\prime}$ básico (o que é possível pelo Lema 2.3.6). Segundo a proposição anterior, existe uma sequência especial de $T^{\prime}$ da forma $\left(T_{1}^{\prime}, \ldots, T_{n}^{\prime}\right)$, onde $T^{\prime}=\oplus_{i=1}^{n} T_{i}$, é a decomposição de $T^{\prime}$ em somandos diretos indecomponíveis. Se $T=\oplus_{i=1}^{k} T_{\ell_{i}}^{\prime}$, então $\left(T_{\ell_{1}}^{\prime}, \ldots, T_{\ell_{k}}^{\prime}\right)$ é uma sequência especial de $T$ se $u<v$ implicar que $\ell_{u}<\ell_{v}$, para $u, v \in\{1, \ldots, k\}$.

Lema 4.1.7. Sejam $K$ um corpo, $A$ uma $K$-álgebra (não necessariamente hereditária). Se $X$ e $Y$ são A-módulos tais que $\operatorname{Hom}_{A}(X, Y)=0$ e existir uma sequência exata da forma

$$
0 \longrightarrow X \stackrel{i}{\longrightarrow} L \stackrel{\phi}{\longrightarrow} Y^{n} \longrightarrow 0
$$

para algum $n \in \mathbb{N}, n \neq 0$, então não existe um epimorfismo $f: L \longrightarrow Y^{n+1}$.

Demonstração. Suponhamos que existe um epimorfismo $f: L \longrightarrow Y^{n+1}$. Então $f i=0$, pois caso contrário existiria um elemento não nulo em $\operatorname{Hom}_{A}(X, Y)$ pela definição de conúcleo existirá um homomorfismo $h: Y^{n} \longrightarrow Y^{n+1}$ tal que $\phi h=f$. Logo, como $\phi$ e $f$ são epimorfismos, temos que $h$ é um epimorfismo o que é uma contradição.

Lema 4.1.8. Sejam A uma K-álgebra hereditária e de dimensão finita sobre um corpo algebricamente fechado $K$ e $T=T_{1} \oplus T_{2}$ um A-módulo inclinante parcial, onde $T_{1}$ e $T_{2}$ são indecomponiveis não isomorfos. Se existir uma sequência exata curta da forma

$$
0 \longrightarrow N \longrightarrow T_{2} \longrightarrow T_{1}^{n} \longrightarrow 0
$$

Então:

1. $\operatorname{Ext}_{A}^{1}\left(N, T_{1}\right)=0$.

2. $\operatorname{Ext}_{A}^{1}\left(N, T_{2}\right)=0$.

3. $\operatorname{Hom}_{A}\left(N, T_{1}\right)=0$ se, e somente se, $\operatorname{dim}_{K} \operatorname{Hom}_{A}\left(T_{2}, T_{1}\right)=n$. 
Demonstração. Para 1 aplicamos o funtor $\operatorname{Hom}_{A}\left(\ldots, T_{1}\right)$ em (4.1) e obtemos a seguinte sequência exata

$$
\cdots \longrightarrow \operatorname{Ext}_{A}^{1}\left(T_{2}, T_{1}\right) \longrightarrow \operatorname{Ext}_{A}^{1}\left(N, T_{1}\right) \longrightarrow \operatorname{Ext}_{A}^{2}\left(T_{1}^{n}, T_{1}\right) \longrightarrow \cdots
$$

Como, por hipótese, $\operatorname{Ext}_{A}^{1}\left(T_{2}, T_{1}\right)=0$ e $\operatorname{Ext}_{A}^{2}\left(T_{1}^{n}, T_{1}\right)=0$, então temos que $\operatorname{Ext}_{A}^{1}\left(N, T_{1}\right)=0$.

Para a demonstração da parte 2, que é análoga à anterior, aplicamos o funtor $\operatorname{Hom}_{A}\left(\ldots, T_{2}\right)$ em (4.1) e obtemos

$$
\cdots \longrightarrow \operatorname{Ext}_{A}^{1}\left(T_{2}, T_{2}\right) \longrightarrow \operatorname{Ext}_{A}^{1}\left(N, T_{2}\right) \longrightarrow \operatorname{Ext}_{A}^{2}\left(T_{1}^{n}, T_{2}\right) \longrightarrow \cdots
$$

Das hipóteses de que $\operatorname{Ext}_{A}^{1}\left(T_{2}, T_{2}\right)=0=\operatorname{Ext}_{A}^{2}\left(T_{1}^{n}, T_{2}\right)=0$, resulta que $\operatorname{Ext}_{A}^{1}\left(N, T_{2}\right)=0$.

Para provar 3 aplicamos $\operatorname{Hom}_{A}\left(\ldots, T_{1}\right)$ em $(4.1)$ e obtemos

$$
0 \longrightarrow \operatorname{Hom}_{A}\left(T_{1}^{n}, T_{1}\right) \longrightarrow \operatorname{Hom}_{A}\left(T_{2}, T_{1}\right) \longrightarrow \operatorname{Hom}_{A}\left(N, T_{1}\right) \longrightarrow 0
$$

$\operatorname{Logo~}_{\operatorname{Hom}}\left(N, T_{1}\right)=0$ se, e somente se, $\operatorname{Hom}_{A}\left(T_{1}^{n}, T_{1}\right) \cong \operatorname{Hom}_{A}\left(T_{2}, T_{1}\right)$. Por outro lado,

$$
\operatorname{dim}_{K} \operatorname{Hom}_{A}\left(T_{1}^{n}, T_{1}\right)=n \operatorname{dim}_{K} \operatorname{End}_{A}\left(T_{1}\right) .
$$

Além disso, pelo Lema 2.3.2, $\operatorname{End}_{A}\left(T_{1}\right) \cong K$.

Portanto, $\operatorname{Hom}_{A}\left(N, T_{1}\right)=0$ se, e somente se, $\operatorname{dim}_{K} \operatorname{Hom}_{A}\left(T_{2}, T_{1}\right)=n$.

Antes de enunciar a próximo lema, notemos que se $\left(T_{1}, \ldots, T_{n}\right)$ é uma sequência especial do $A$-módulo inclinante $T$ associada a sistema estratificante $\left(X_{1}, \ldots, X_{2}\right)$, então $X_{1}=T_{1}$.

Lema 4.1.9. Sejam A uma álgebra hereditária e $T=T_{1} \oplus T_{2}$ um A-módulo inclinante parcial com $T_{1}$ e $T_{2}$ módulos indecomponíveis não isomorfos. Se existe um monomorfismo $f: T_{2} \rightarrow T_{1}$, então $\left(T_{1}, T_{2}\right)$ não é uma sequência especial de $T$.

Demonstração. Suponhamos que $\left(T_{1}, T_{2}\right)$ seja uma sequência especial de $T$ e que $f: T_{2} \rightarrow T_{1}$ é um monomorfismo. Então, pela definição, existe um sistema estratificante $X=\left(X_{1}, X_{2}\right)$ com $X_{1}=T_{1}$, tal que $\mathcal{F}(X) \cap \mathcal{Y}(X)=\operatorname{add} T$ e uma sequência exata da forma

$$
0 \longrightarrow X_{2} \stackrel{\alpha}{\longrightarrow} T_{2} \longrightarrow X_{1}^{n} \longrightarrow 0
$$

Portanto $f \alpha: X_{2} \longrightarrow X_{1}$ é um monomorfismo, $\log$ $\operatorname{Hom}_{A}\left(X_{2}, X_{1}\right) \neq 0$, o que contraria a hipótese de que $\operatorname{Hom}_{A}\left(X_{2}, X_{1}\right)=0$.

Corolário 4.1.10. Sejam $A$ uma $K$-álgebra hereditária, $T=T_{1} \oplus T_{2}$ um A-módulo inclinante parcial, com $T_{1}$ e $T_{2}$ A-módulos indecomponíveis, e $\left(T_{1}, T_{2}\right)$ uma sequência especial de $T$. Então: 
1. Todos os morfismos não nulos em $\operatorname{Hom}_{A}\left(T_{2}, T_{1}\right)$ são epimorfismos.

2. Existe um epimorfismo da forma $f: T_{2} \rightarrow T_{1}^{n}$, com $n=\operatorname{dim}_{K} \operatorname{Hom}_{A}\left(T_{2}, T_{1}\right)$.

3. O máximo inteiro $n$ com a propriedade de que existe um epimorfismo $f: T_{2} \longrightarrow T_{1}^{n}$ é $n=\operatorname{dim}_{K} \operatorname{Hom}_{A}\left(T_{2}, T_{1}\right)$.

Demonstração. O Lema 4.1 .9 garante que não existem monomorfismos em $\operatorname{Hom}_{A}\left(T_{2}, T_{1}\right)$. Então, pelo Lema 2.3.2, qualquer elemento não nulo em $\operatorname{Hom}_{A}\left(T_{2}, T_{1}\right)$ é um epimorfismo. Por outro lado, desde que $\left(T_{1}, T_{2}\right)$ é uma sequência especial de $T$, existe uma sequência exata da forma

$$
0 \longrightarrow X_{2} \longrightarrow T_{2} \longrightarrow T_{1}^{m} \longrightarrow 0 .
$$

Então, pelo Lema 4.1.8, $m=\operatorname{dim}_{K} \operatorname{Hom}_{A}\left(T_{2}, T_{1}\right)$. Se $m=0$, não temos mais nada a provar. Se $m \neq 0$, pelo Lema 4.1.7, $m$ satisfaz as condições requeridas.

Notemos que se $T=T_{1} \oplus T_{2}$ é um $A$-módulo inclinante parcial, $\left(T_{1}, T_{2}\right)$ é uma sequência especial de $T$ e $\operatorname{Hom}_{A}\left(T_{2}, T_{1}\right)=0$, então $\left(T_{1}, T_{2}\right)$ é o sistema estratificante associado a $\left(T_{1}, T_{2}\right)$.

Lema 4.1.11. Sejam $A$ uma $K$-álgebra hereditária, $T=T_{1} \oplus T_{2}$ um $A$-módulo inclinante parcial e $T_{1}$ e $T_{2}$ módulos indecomponíveis não isomorfos. Se existe uma sequência exata curta da forma

$$
0 \longrightarrow N \longrightarrow T_{2} \longrightarrow T_{1}^{n} \longrightarrow 0
$$

$e \operatorname{Hom}_{A}\left(N, T_{1}\right)=0$. Então:

1. $\operatorname{Ext}_{A}^{1}(N, N)=0$.

2. As seguintes condições são equivalentes:

(a) $N$ é um A-módulo indecomponível.

(b) $\operatorname{dim}_{K} \operatorname{Hom}_{A}\left(N, T_{2}\right)=1$.

(c) $\operatorname{Hom}_{A}\left(T_{1}, T_{2}\right)=0$.

Demonstração. Aplicamos o funtor $\operatorname{Hom}_{A}(N$, _ $)$ na sequência (4.1) e como, pelo Lema 4.1.8, $\operatorname{Ext}_{A}^{1}\left(N, T_{1}\right)=0$ obtemos a seguinte sequência exata

$$
0 \rightarrow \operatorname{End}_{A}(N) \rightarrow \operatorname{Hom}_{A}\left(N, T_{2}\right) \rightarrow \operatorname{Hom}_{A}\left(N, T_{1}\right) \rightarrow \operatorname{Ext}_{A}^{1}(N, N) \rightarrow 0 .
$$

Por hipótese $\operatorname{Hom}_{A}\left(N, T_{1}\right)=0$, então, usando a sequência exata acima, temos que $\operatorname{Ext}_{A}^{1}(N, N)=$ 0 (que é nossa primeira afirmação) e que $\operatorname{End}_{A}(N) \cong \operatorname{Hom}_{A}\left(N, T_{2}\right)$.

Vamos provar que $(a \Leftrightarrow b)$. Para isto notemos que, o fato de que $N$ não tem auto-extensões junto com o Lema 2.3.3, implicam que $N$ é indecomponível se, e somente se, $\operatorname{End}_{A}(N) \cong K$. 
Mas, por outro lado $\operatorname{End}_{A}(N) \cong \operatorname{Hom}_{A}\left(N, T_{2}\right)$, então podemos concluir que, $N$ é indecomponível se, e somente se, $\operatorname{dim}_{K} \operatorname{Hom}_{A}\left(N, T_{2}\right)=1$.

Para mostrar $(b \Leftrightarrow c)$, aplicamos o funtor $\operatorname{Hom}_{A}\left(\ldots, T_{2}\right)$ em $(4.1)$ e obtemos a sequência exata curta

$$
0 \longrightarrow \operatorname{Hom}_{A}\left(T_{1}^{n}, T_{2}\right) \longrightarrow \operatorname{End}_{A}\left(T_{2}\right) \longrightarrow \operatorname{Hom}_{A}\left(N, T_{2}\right) \longrightarrow 0
$$

Como $T_{2}$ é um $A$-módulo indecomponível e sem auto-extensões, pelo Lema 2.3.3, temos que $\operatorname{dim}_{K} \operatorname{End}_{A}\left(T_{2}\right)=1$. Portanto, usando a sequência exata acima, é possível afirmar que

$$
\operatorname{dim}_{K} \operatorname{Hom}_{A}\left(N, T_{2}\right)=1 \text { se, e somente se, } \operatorname{Hom}_{A}\left(T_{1}, T_{2}\right)=0 .
$$

Teorema 4.1.12. Sejam $K$ um corpo algebricamente fechado, $A$ uma $K$-álgebra hereditária e $T=T_{1} \oplus T_{2}$ um A-módulo inclinante parcial com $T_{1}$ e $T_{2}$ A-módulos indecomponíveis. Então $\left(T_{1}, T_{2}\right)$ é uma sequência especial de $T$ se, e somente se, os morfismos não nulos em $\operatorname{Hom}_{A}\left(T_{2}, T_{1}\right)$ são epimorfismos e existe um epimorfismo da forma $f: T_{2} \longrightarrow T_{1}^{n}$, onde $n=$ $\operatorname{dim}_{K} \operatorname{Hom}_{A}\left(T_{2}, T_{1}\right)$. Neste caso, $\left(T_{1}, T_{2}\right)$ é a sequência especial de $T$ associada ao sistema estratificante $\left(T_{1}\right.$, Nuc $\left.f\right)$.

Demonstração. Se $\left(T_{1}, T_{2}\right)$ é uma sequência especial de $T$ a tese segue diretamente do Corolário 4.1.10. Para a demonstração do recíproco, suponhamos que existe um epimorfismo da forma $f: T_{2} \longrightarrow T_{1}^{n}$, onde $n=\operatorname{dim}_{K} \operatorname{Hom}_{A}\left(T_{2}, T_{1}\right)$. Se $n=0$, não temos nada a provar. Suponhamos que $n \neq 0$. Seja $N=$ Nuc $f$. Então, pelo Lema 4.1.8, $\operatorname{Hom}_{A}\left(N, T_{1}\right)=0$ e $\operatorname{Ext}_{A}^{1}\left(N, T_{1}\right)=0$.

Por outro lado, Lema 4.1.3, $\operatorname{Hom}_{A}\left(T_{1}, T_{2}\right)=0$. Logo, pelo Lema 4.1.11, $N$ é um $A$-módulo indecomponível e $\operatorname{Ext}_{A}^{1}(N, N)=0$. Assim temos que $\left(T_{1}, N\right)$ é um sistema estratificante. Por outro lado temos que $\operatorname{Ext}_{A}^{1}\left(T_{1}, T_{1} \oplus T_{2}\right)=0$, pois $T$ é inclinante parcial e, pelo Lema 4.1.8, que $\operatorname{Ext}_{A}^{1}\left(N, T_{1} \oplus T_{2}\right)=0$. Assim podemos afirmar que $\mathcal{F}\left(T_{1}, N\right) \cap \mathcal{Y}\left(T_{1}, N\right)=$ add $T$, e portanto que $\left(T_{1}, N\right)$ é o sistema estratificante associado à sequência especial $\left(T_{1}, T_{2}\right)$ de $T$.

Lema 4.1.13. Sejam A uma $K$-álgebra hereditária, $T=T_{1} \oplus T_{2}$ um A-módulo inclinante parcial, com A-módulos $T_{1}$ e $T_{2}$ indecomponiveis de forma que $\left(T_{1}, T_{2}\right)$ é uma sequência especial de $T$, associada ao sistema estratificante $\left(X_{1}, X_{2}\right)$. Se $\operatorname{Hom}_{A}\left(T_{2}, T_{1}\right) \neq 0$, então $\operatorname{Hom}_{A}\left(X_{1}, X_{2}\right)=$ 0 e $\operatorname{dim}_{K} \operatorname{Ext}_{A}^{1}\left(X_{1}, X_{2}\right)=\operatorname{dim}_{K} \operatorname{Hom}_{A}\left(T_{2}, T_{1}\right)$.

Demonstração. Como $\operatorname{Hom}_{A}\left(T_{2}, T_{1}\right) \neq 0$ indiquemos por $n_{1}=\operatorname{dim}_{K} \operatorname{Hom}_{A}\left(T_{2}, T_{1}\right) \geq 1$. A hipótese de $\left(T_{1}, T_{2}\right)$ ser uma sequência especial associada ao sistema estratificante $\left(X_{1}, X_{2}\right)$, $X_{1}=T_{1}$, implica, pelo Teorema 4.1.12, que existe uma sequência exata curta da forma

$$
0 \longrightarrow X_{2} \longrightarrow T_{2} \longrightarrow T_{1}^{n_{1}} \longrightarrow 0
$$


Aplicando o funtor $\operatorname{Hom}_{A}\left(T_{1}, \ldots\right)$ à sequência exata acima, obtemos que

$$
0 \rightarrow \operatorname{Hom}_{A}\left(T_{1}, X_{2}\right) \rightarrow \operatorname{Hom}_{A}\left(T_{1}, T_{2}\right) \rightarrow \operatorname{Hom}_{A}\left(T_{1}, T_{1}^{n}\right) \rightarrow \operatorname{Ext}_{A}^{1}\left(T_{1}, X_{2}\right) \rightarrow 0 .
$$

Porém, como consequência do Lema 4.1.3, temos que $\operatorname{Hom}_{A}\left(T_{1}, T_{2}\right)=0$. Portanto da última sequência resulta que $\operatorname{Hom}_{A}\left(X_{1}, X_{2}\right)=\operatorname{Hom}_{A}\left(T_{1}, X_{2}\right)=0$ e que $\operatorname{dim}_{K} \operatorname{Ext}^{1}\left(X_{1}, X_{2}\right)=$ $\operatorname{dim}_{K} \operatorname{Hom}\left(T_{1}, T_{1}\right)^{n_{1}}=n_{1}$.

Proposição 4.1.14. Sejam $K$ um corpo algebricamente fechado, A uma K-álgebra hereditária e $T=T_{1} \oplus T_{2} \oplus T_{3}$ um A-módulo inclinante parcial básico tal que $T_{1}, T_{2}$ e $T_{3}$ são A-módulos indecomponiveis. Se $\left(T_{1}, T_{2}\right)$ é uma sequência especial de $T_{1} \oplus T_{2}$ associada ao sistema estratificante $\left(X_{1}, X_{2}\right)$, onde $X_{2} \neq T_{2}$, e existem sequências exatas da forma

$$
\begin{gathered}
0 \longrightarrow K_{1} \longrightarrow T_{3} \longrightarrow T_{1}^{n_{1}} \longrightarrow 0 \\
0 \longrightarrow K_{2} \longrightarrow K_{1} \longrightarrow X_{2}^{n_{2}} \longrightarrow 0
\end{gathered}
$$

onde $n_{1}=\operatorname{dim}_{K} \operatorname{Hom}_{A}\left(T_{3}, T_{1}\right)$ e $n_{2}=\operatorname{dim}_{K} \operatorname{Hom}_{A}\left(T_{3}, T_{2}\right)$. Então $\left(T_{1}, T_{2}, T_{3}\right)$ é uma sequência especial de $T$ associada ao sistema estratificante $\left(X_{1}, X_{2}, K_{2}\right)$.

Demonstração. Primeiro vamos aplicar o funtor $\operatorname{Hom}_{A}\left(\ldots, T_{1}\right)$ em $(4.3)$ e obtemos

$$
\begin{aligned}
& 0 \longrightarrow \operatorname{Hom}_{A}\left(X_{2}^{n_{2}}, T_{1}\right)\left.\left.\longrightarrow \operatorname{Hom}_{A}\left(K_{1}, T_{1}\right) \longrightarrow \operatorname{Hom}_{A}\left(K_{2}, T_{1}\right) \longrightarrow \operatorname{Ext}_{A}^{1}\left(K_{1}, T_{1}\right) \longrightarrow K_{1}\right) \longrightarrow T_{1}\right) \longrightarrow 0 \\
& \operatorname{Ext}_{A}^{1}\left(X_{2}^{n_{2}}, T_{1}\right) \longrightarrow \operatorname{Ext}_{A}^{1} \longrightarrow
\end{aligned}
$$

Como $\left(X_{1}, X_{2}\right)$ é um sistema estratificante associado a sequência especial $\left(T_{1}, T_{2}\right)$ de $T_{1} \oplus T_{2}$, então $X_{1}=T_{1}, \operatorname{Hom}_{A}\left(X_{2}, T_{1}\right)=0$ e $\operatorname{Ext}_{A}^{1}\left(X_{2}, T_{1}\right)=0$. Logo, da sequência acima, podemos concluir que

$$
\operatorname{Hom}_{A}\left(K_{1}, T_{1}\right) \cong \operatorname{Hom}_{A}\left(K_{2}, T_{1}\right) \text { e que } \operatorname{Ext}_{A}^{1}\left(K_{1}, T_{1}\right) \cong \operatorname{Ext}_{A}^{1}\left(K_{2}, T_{1}\right)
$$

Aplicamos agora o funtor $\operatorname{Hom}_{A}\left(\ldots, T_{1}\right)$ em (4.2) e obtemos

$$
\begin{aligned}
& 0 \longrightarrow \operatorname{Hom}_{A}\left(T_{1}^{n_{1}}, T_{1}\right)\left.\longrightarrow \operatorname{Hom}_{A}\left(T_{3}, T_{1}\right) \longrightarrow \operatorname{Hom}_{A}\left(K_{1}, T_{1}\right) \longrightarrow \operatorname{Ext}_{A}^{1}\left(T_{3}, T_{1}\right) \longrightarrow K_{1}, T_{1}\right) \longrightarrow 0 . \\
& \operatorname{Ext}_{A}^{1}\left(T_{1}^{n_{1}}, T_{1}\right) \longrightarrow \operatorname{Ext}_{A} \longrightarrow 0 .
\end{aligned}
$$

Como $\operatorname{Ext}_{A}^{1}\left(T_{3}, T_{1}\right)=0$, então $\operatorname{Ext}_{A}^{1}\left(K_{1}, T_{1}\right)=0$ e, usando (4.4), temos que

$$
\operatorname{Ext}_{A}^{1}\left(K_{2}, T_{1}\right)=0 \text { e } \operatorname{Hom}_{A}\left(K_{2}, T_{1}\right)=0 \Leftrightarrow \operatorname{dim}_{K} \operatorname{Hom}_{A}\left(T_{3}, T_{1}\right)=n_{1} .
$$


Agora aplicamos $\operatorname{Hom}_{A}\left(\ldots, T_{3}\right)$ em (4.2) e obtemos

$$
\cdots \longrightarrow \operatorname{Ext}_{A}^{1}\left(T_{1}^{n_{1}}, T_{3}\right) \longrightarrow \operatorname{Ext}_{A}^{1}\left(T_{3}, T_{3}\right) \longrightarrow \operatorname{Ext}_{A}^{1}\left(K_{1}, T_{3}\right) \longrightarrow 0
$$

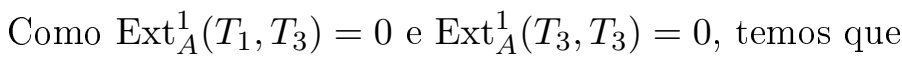

$$
\operatorname{Ext}_{A}^{1}\left(K_{1}, T_{3}\right)=0
$$

Aplicando $\operatorname{Hom}_{A}\left(\ldots, T_{3}\right)$ em (4.3) e obtemos

$$
\cdots \longrightarrow \operatorname{Ext}_{A}^{1}\left(X_{2}, T_{3}\right) \longrightarrow \operatorname{Ext}_{A}^{1}\left(K_{1}, T_{3}\right) \longrightarrow \operatorname{Ext}_{A}^{1}\left(K_{2}, T_{3}\right) \longrightarrow 0
$$

Da sequência exata acima e (4.6) concluímos que

$$
\operatorname{Ext}_{A}^{1}\left(K_{2}, T_{3}\right)=0
$$

Aplicamos $\operatorname{Hom}_{A}\left(K_{2}, \ldots\right)$ em (4.2) e temos

$$
\begin{aligned}
& 0 \longrightarrow \operatorname{Hom}_{A}\left(K_{2}, K_{1}\right) \longrightarrow \operatorname{Hom}_{A}\left(K_{2}, T_{3}\right) \longrightarrow \operatorname{Hom}_{A}\left(K_{2}, T_{1}^{n_{1}}\right) \longrightarrow \operatorname{Ext}_{A}^{1}\left(K_{2}, T_{3}\right) \longrightarrow \operatorname{Ext}_{A}^{1}\left(K_{2}, T_{1}^{n_{1}}\right) \longrightarrow 0 \\
& \operatorname{Ext}_{A}^{1}\left(K_{2}, K_{1}\right) \longrightarrow{ }_{0} \longrightarrow
\end{aligned}
$$

Mas, usando (4.7) e (4.6), temos que

$$
\operatorname{Ext}_{A}^{1}\left(K_{2}, K_{1}\right)=0 \text { e que } \operatorname{Hom}_{A}\left(K_{2}, K_{1}\right) \cong \operatorname{Hom}_{A}\left(K_{2}, T_{3}\right)
$$

Aplicamos $\operatorname{Hom}_{A}\left(K_{2}, \ldots\right)$ em (4.3)

$$
\begin{aligned}
& 0 \longrightarrow \operatorname{Hom}_{A}\left(K_{2}, K_{2}\right) \longrightarrow \operatorname{Hom}_{A}\left(K_{2}, K_{1}\right) \longrightarrow \operatorname{Hom}_{A}\left(K_{2}, X_{2}^{n_{2}}\right) \longrightarrow \\
& \operatorname{Ext}_{A}^{1}\left(K_{2}, K_{2}\right) \longrightarrow \operatorname{Ext}_{A}^{1}\left(K_{2}, K_{1}\right) \longrightarrow \operatorname{Ext}_{A}^{1}\left(K_{2}, X_{2}^{n_{2}}\right) \longrightarrow 0
\end{aligned}
$$

Usando (4.9), na sequência exata acima, temos que

$$
\operatorname{Ext}_{A}^{1}\left(K_{2}, X_{2}\right)=0
$$

Aplicamos $\operatorname{Hom}_{A}\left(\ldots, X_{2}\right)$ em (4.3)

$$
\begin{aligned}
0 \longrightarrow \operatorname{Hom}_{A}\left(X_{2}^{n_{2}}, X_{2}\right) & \longrightarrow \operatorname{Hom}_{A}\left(K_{1}, X_{2}\right) \longrightarrow \operatorname{Hom}_{A}\left(K_{2}, X_{2}\right) \longrightarrow \\
\operatorname{Ext}_{A}^{1}\left(X_{2}^{n_{2}}, X_{2}\right) & \longrightarrow \operatorname{Ext}_{A}^{1}\left(K_{1}, X_{2}\right) \longrightarrow \operatorname{Ext}_{A}^{1}\left(K_{2}, X_{2}\right) \longrightarrow 0
\end{aligned}
$$

Como $\left(X_{1}, X_{2}\right)$ é um sistema estratificante, então $X_{2}$ não tem auto-extensões e por outro lado, de (4.11), temos que

$$
\operatorname{Ext}_{A}^{1}\left(K_{1}, X_{2}\right)=0 \text { e que } \operatorname{Hom}_{A}\left(K_{2}, X_{2}\right)=0 \Leftrightarrow \operatorname{dim}_{K} \operatorname{Hom}_{A}\left(K_{1}, X_{2}\right)=n_{2} \text {. }
$$


Agora aplicamos $\operatorname{Hom}_{A}\left(\ldots, X_{2}\right)$ em (4.2) e obtemos

$$
\begin{aligned}
& 0 \longrightarrow \operatorname{Hom}_{A}\left(T_{1}^{n_{1}}, X_{2}\right) \longrightarrow \operatorname{Hom}_{A}\left(T_{3}, X_{2}\right) \longrightarrow \operatorname{Hom}_{A}\left(K_{1}, X_{2}\right) \longrightarrow \operatorname{Ext}_{A}^{1}\left(T_{3}, X_{2}\right) \longrightarrow \operatorname{Ext}_{A}^{1}\left(K_{1}, X_{2}\right) \longrightarrow 0 . \\
& \operatorname{Ext}_{A}^{1}\left(T_{1}^{n_{1}}, X_{2}\right) \longrightarrow{ }^{2} \longrightarrow
\end{aligned}
$$

Seja $n=\operatorname{dim}_{K} \operatorname{Hom}_{A}\left(T_{2}, T_{1}\right)$. Como, por hipótese $X_{2} \nsucceq T_{2}$, então $n \neq 0$. Assim, pelo Lema 4.1.13, $\operatorname{Hom}_{A}\left(T_{1}, X_{2}\right)=0$ e $\operatorname{dim}_{K} \operatorname{Ext}_{A}^{1}\left(T_{1}, X_{2}\right)=n$. Por outro lado, de (4.12), temos que $\operatorname{Ext}_{A}^{1}\left(K_{1}, X_{2}\right)=0$. Portanto, da sequência exata acima temos que

$$
\begin{aligned}
\operatorname{dim}_{K} \operatorname{Hom}_{A}\left(T_{3}, X_{2}\right) & =\operatorname{dim}_{K} \operatorname{Hom}_{A}\left(K_{1}, X_{2}\right)-\operatorname{dim}_{K} \operatorname{Ext}_{A}^{1}\left(T_{1}^{n_{1}}, X_{2}\right)+\operatorname{dim}_{K} \operatorname{Ext}_{A}^{1}\left(T_{3}, X_{2}\right) \\
& =\operatorname{dim}_{K} \operatorname{Hom}_{A}\left(K_{1}, X_{2}\right)+\operatorname{dim}_{K} \operatorname{Ext}_{A}^{1}\left(T_{3}, X_{2}\right)-n n_{1} .
\end{aligned}
$$

Mas, por outro lado, pelo Teorema 4.1.12, existe uma sequência exata da forma

$$
0 \longrightarrow X_{2} \longrightarrow T_{2} \longrightarrow T_{1}^{n} \longrightarrow 0
$$

Se aplicamos o funtor $\operatorname{Hom}_{A}\left(T_{3}, \ldots\right)$, na sequência acima, obtemos

$$
0 \rightarrow \operatorname{Hom}_{A}\left(T_{3}, X_{2}\right) \rightarrow \operatorname{Hom}_{A}\left(T_{3}, T_{2}\right) \rightarrow \operatorname{Hom}_{A}\left(T_{3}, T_{1}^{n}\right) \rightarrow \operatorname{Ext}_{A}^{1}\left(T_{3}, X_{2}\right) \rightarrow 0 .
$$

Assim, em termos de dimensão, temos que

$$
\operatorname{dim}_{K} \operatorname{Hom}_{A}\left(T_{3}, T_{2}\right)=\operatorname{dim}_{K} \operatorname{Hom}_{A}\left(T_{3}, X_{2}\right)+\operatorname{dim}_{K} \operatorname{Hom}_{A}\left(T_{3}, X_{1}^{n}\right)-\operatorname{dim}_{K} \operatorname{Ext}_{A}^{1}\left(T_{3}, X_{2}\right) .
$$

Notemos que $\operatorname{dim}_{K} \operatorname{Hom}_{A}\left(T_{3}, X_{1}^{n}\right)=n n_{1}$. Agora, sustituindo (4.13) em (4.15), temos que

$$
\operatorname{dim}_{K} \operatorname{Hom}_{A}\left(T_{3}, T_{2}\right)=\operatorname{dim}_{K} \operatorname{Hom}_{A}\left(K_{1}, X_{2}\right) .
$$

Mas, usando (4.12) e (4.16) podemos afirmar que

$$
\operatorname{Hom}_{A}\left(K_{2}, X_{2}\right)=0 \Leftrightarrow \operatorname{dim}_{K} \operatorname{Hom}_{A}\left(T_{3}, T_{2}\right)=n_{2} .
$$

Até agora, temos demonstrado que

$$
\begin{aligned}
& \operatorname{Hom}_{A}\left(K_{2}, X_{1}\right)=0, \\
& \operatorname{Hom}_{A}\left(K_{2}, X_{2}\right)=0, \\
& \operatorname{Ext}_{A}^{1}\left(K_{2}, X_{1}\right)=0, \\
& \operatorname{Ext}_{A}^{1}\left(K_{2}, X_{2}\right)=0 .
\end{aligned}
$$

Diante disto, para demonstrar que $\left(X_{1}, X_{2}, K_{2}\right)$ é um sistema estratificante, é suficiente verificar que o módulo $K_{2}$ não tem auto-extensões e que é um $A$-módulo indecomponível.

Como $\operatorname{Hom}_{A}\left(K_{2}, X_{1}\right)=0$, segundo (4.5), e $\operatorname{Ext}_{A}^{1}\left(K_{2}, T_{3}\right)=0$, por (4.7), então, usando (4.8), 
podemos afirmar que

$$
\operatorname{Ext}_{A}^{1}\left(K_{2}, K_{1}\right)=0 \text {. }
$$

Usando (4.9), (4.17) e (4.18) na sequência exata em (4.10), podemos dizer que

$$
\operatorname{Ext}_{A}^{1}\left(K_{2}, K_{2}\right)=0 \text { e que } \operatorname{Hom}_{A}\left(K_{2}, K_{2}\right) \cong \operatorname{Hom}_{A}\left(K_{2}, K_{1}\right) \cong \operatorname{Hom}_{A}\left(K_{2}, T_{3}\right) \text {. }
$$

Agora, usando o Corolário 2.3.3 e a cadeia de isomorfismos acima em (4.19), afirmamos que

$$
K_{2} \text { é indecomponível } \Leftrightarrow \operatorname{dim}_{K} \operatorname{Hom}_{A}\left(K_{2}, T_{3}\right)=1 \text {. }
$$

Por outro lado, aplicando $\operatorname{Hom}_{A}\left(\ldots, T_{3}\right)$ em (4.14), temos que

$$
\begin{aligned}
0 \longrightarrow \operatorname{Hom}_{A}\left(T_{1}^{n}, T_{3}\right) & \longrightarrow \operatorname{Hom}_{A}\left(T_{2}, T_{3}\right) \longrightarrow \operatorname{Hom}_{A}\left(X_{2}, T_{3}\right) \longrightarrow \operatorname{Ext}_{A}^{1}\left(T_{2}, T_{3}\right) \longrightarrow \operatorname{Ext}_{A}^{1}\left(X_{2}, T_{3}\right) \longrightarrow 0 \\
\operatorname{Ext}_{A}^{1}\left(T_{1}^{n}, T_{3}\right) \longrightarrow & \longrightarrow
\end{aligned}
$$

Como $\operatorname{Ext}_{A}^{1}\left(T_{2}, T_{3}\right)=0$, então

$$
\operatorname{Ext}_{A}^{1}\left(X_{2}, T_{3}\right)=0
$$

Usando as sequências em (4.2) e (4.3) construímos seguinte o diagrama de pust-out

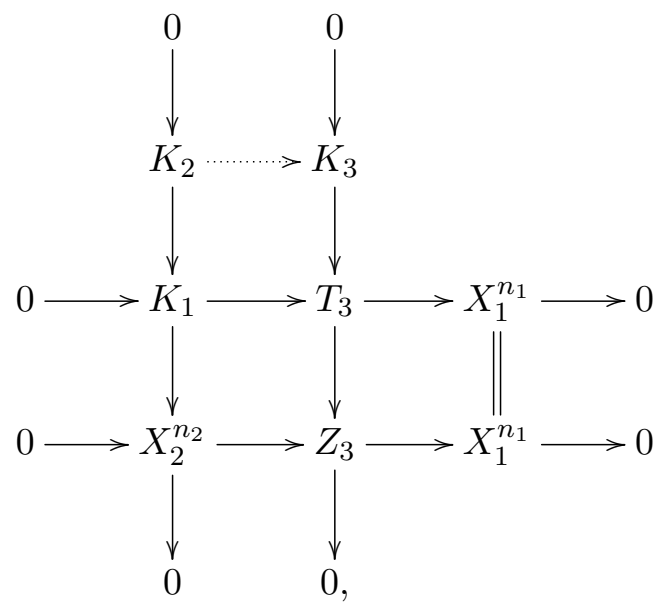

onde, como consequência do Lema da serpente, a linha pontilhada é um isomorfismo.

Aplicamos em $0 \longrightarrow X_{2}^{n_{2}} \longrightarrow Z_{3} \longrightarrow X_{1}^{n_{1}} \longrightarrow 0$, que é a segunda sequência horizontal no diagrama acima, o funtor $\operatorname{Hom}_{A}\left(\ldots, T_{3}\right)$ e obtemos

$$
\begin{aligned}
& 0 \longrightarrow \operatorname{Hom}_{A}\left(X_{1}^{n_{1}}, T_{3}\right) \longrightarrow \operatorname{Hom}_{A}\left(Z_{3}, T_{3}\right) \longrightarrow \operatorname{Hom}_{A}\left(X_{2}^{n_{2}}, T_{3}\right) \longrightarrow \operatorname{Ext}_{A}^{1}\left(Z_{3}, T_{3}\right) \longrightarrow \operatorname{Ext}_{A}^{1}\left(X_{2}^{n_{2}}, T_{3}\right) \longrightarrow 0 . \\
& \operatorname{Ext}_{A}^{1}\left(X_{1}^{n_{1}}, T_{3}\right) \longrightarrow{ }^{2} \longrightarrow
\end{aligned}
$$

Como $\operatorname{Ext}_{A}^{1}\left(X_{2}, T_{3}\right)=0$, por (4.22), e $\operatorname{Ext}_{A}^{1}\left(X_{1}, T_{3}\right)=\operatorname{Ext}_{A}^{1}\left(T_{1}, T_{3}\right)=0$, então

$$
\operatorname{Ext}_{A}^{1}\left(Z_{3}, T_{3}\right)=0
$$


Agora aplicando $\operatorname{Hom}_{A}\left(T_{3}\right)$ na segunda sequência vertical do diagrama acima, e usando (4.24) temos que

$$
0 \longrightarrow \operatorname{Hom}_{A}\left(Z_{3}, T_{3}\right) \longrightarrow \operatorname{Hom}_{A}\left(T_{3}, T_{3}\right) \longrightarrow \operatorname{Hom}_{A}\left(K_{2}, T_{3}\right) \longrightarrow 0 .
$$

Pelo Lema 2.3.2, $\operatorname{End}_{A}\left(T_{3}\right) \cong K$, logo, usando (4.20) e a sequência acima, afirmamos que

$$
K_{2} \text { é indecomponível } \Leftrightarrow \operatorname{Hom}_{A}\left(Z_{3}, T_{3}\right)=0 .
$$

Por outro lado, de (4.23) e (4.22), temos que

$$
\operatorname{Hom}_{A}\left(Z_{3}, T_{3}\right)=0 \Leftrightarrow\left(\operatorname{Hom}_{A}\left(X_{2}, T_{3}\right)=0 \text { e } \operatorname{Hom}_{A}\left(X_{1}, T_{3}\right)=0\right)
$$

Finalmente, de (4.21) e (4.22), temos que

$$
\operatorname{Hom}_{A}\left(T_{2}, T_{3}\right)=0 \Leftrightarrow\left(\operatorname{Hom}_{A}\left(X_{2}, T_{3}\right)=0 \text { e } \operatorname{Hom}_{A}\left(X_{1}, T_{3}\right)=0\right) .
$$

Para verificar que $K_{2}$ é undecomponível devemos considerar vários casos. Suponhamos que $n_{2}=0$. Então $K_{2} \cong K_{1}$. Além disso, se $n_{1} \neq 0$, então $\operatorname{Hom}_{A}\left(T_{1}, T_{3}\right)=0$, pelo Lema 4.1.3, e, segundo o Lema 4.1.11, $K_{1}$ seria um $A$-módulo indecomponível. No caso contrário, se $n_{1}=0$, então $K_{1} \cong T_{3}$ e, portanto, é um $A$-módulo indecomponível.

Suponhamos que $n_{2} \neq 0$. Então segue do Lema 4.1.3 que $\operatorname{Hom}_{A}\left(T_{2}, T_{3}\right)=0$. Logo de (4.27), (4.26), e (4.25) temos que $K_{2}$ é um $A$-módulo indecomponível.

Lema 4.1.15. [15] Sejam $A$ uma álgebra de Artin e $X=\left\{X_{1}, \ldots, X_{t}\right\}$ um conjunto finito de $A$-módulos com a propriedade de que $\operatorname{Ext}_{A}^{1}\left(X_{i}, X_{j}\right)=0$, sempre que $i \geq j$. Então $M \in \mathcal{F}(X)$ se, e somente se, $M$ tem uma cadeia de submódulos da forma

$$
0=M_{n+1} \subseteq M_{n} \subseteq \ldots \subseteq M_{2} \subseteq M_{1}=M
$$

com $M_{i} / M_{i+1}$ isomorfo a soma direta de copias de $X_{i}$, para todo $1 \leq i \leq n$.

Proposição 4.1.16. Sejam $K$ um corpo algebricamente fechado, A uma K-álgebra hereditária, $T=\bigoplus_{j=1}^{t} T_{j}$ um A-módulo inclinante parcial e $X=\left(X_{1}, \ldots, X_{t}\right)$ um sistema estratificante tal que $\left(T_{1}, \ldots, T_{t}\right)$ é uma sequência especial de $T$ associada a $X$. Então, para cada $j=1, \ldots, t$, 
existem sequências exatas da forma

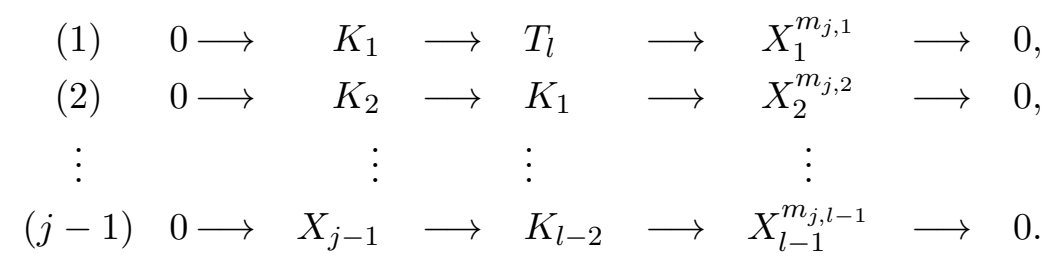

Demonstração. Seja $j \in\{1, \ldots, t\}$ fixo. Pelas hipóteses existe uma sequência exata da forma

$$
0 \longrightarrow X_{j} \longrightarrow T_{j} \longrightarrow Z_{j} \longrightarrow 0, \text { onde } Z_{j} \in \mathcal{F}\left(X_{1}, \ldots, X_{j-1}\right) \text {. }
$$

Portanto $T_{j} \in \mathcal{F}\left(X_{1}, \ldots, X_{j}\right)$ e $\left[T_{j}: X_{j}\right]=1$. Logo, segundo o Lema anterior, existe uma cadeia de submódulos de $T_{j}$ da forma

$$
0=M_{j+1} \subseteq M_{j} \subseteq \ldots \subseteq M_{2} \subseteq M_{1}=T_{j}
$$

onde $M_{i} / M_{i+1} \cong X_{i}^{\alpha_{i}}$, para todo $i=1, \ldots, j$. Para $i=1, M_{1} / M_{2} \cong X_{1}^{\alpha_{1}}$, ou seja, temos uma sequência exata da forma

$$
0 \longrightarrow M_{2} \longrightarrow T_{i} \longrightarrow X_{1}^{\alpha_{1}} \longrightarrow 0 .
$$

Para $i=2, M_{2} / M_{3} \cong X_{2}^{\alpha_{2}}$, portanto é possível construir a sequência exata

$$
0 \longrightarrow M_{3} \longrightarrow M_{2} \longrightarrow X_{2}^{\alpha_{2}} \longrightarrow 0
$$

Continuamos este procedimento até $i=j-1$, onde construímos a sequência

$$
0 \longrightarrow M_{j} \longrightarrow M_{j-1} \longrightarrow X_{j-1}^{\alpha_{j-1}} \longrightarrow 0
$$

Por outro lado, notemos que $M_{j} \cong X_{j}^{\alpha_{j}}$. Mais ainda, o fato de que $\left[T_{j}: X_{j}\right]=1$ implica que $M_{j} \cong X_{j}$.

Desta forma, temos construído a família de sequências exatas desejada.

No caso particular em que $T=T_{1} \oplus T_{2} \oplus T_{3}$ e $\left(T_{1}, T_{2}, T_{3}\right)$ é uma sequência especial de $T$ associada ao sistema estratificante $X=\left(X_{1}, X_{2}, X_{3}\right)$, a proposição acima garante a existência das sequências exatas

$$
\begin{aligned}
& \text { (1) } 0 \longrightarrow K_{1} \longrightarrow T_{3} \longrightarrow X_{1}^{m_{3,1}} \longrightarrow 0 \\
& (2) 0 \longrightarrow X_{3} \longrightarrow K_{1} \longrightarrow X_{2}^{m_{3,2}} \longrightarrow 0 .
\end{aligned}
$$

Além disso, se $X_{2} \nsucceq T_{2}$, então de (4.5) e (4.12) podemos afirmar que $m_{3,1}=\operatorname{dim}_{K} \operatorname{Hom}_{A}\left(T_{3}, T_{1}\right)$ e que $m_{3,2}=\operatorname{dim}_{K} \operatorname{Hom}_{A}\left(T_{3}, T_{2}\right)$. Potanto, temos demostrado o seguinte teorema. 
Teorema 4.1.17. Sejam $K$ um corpo algebricamente fechado, $A$ uma $K$-álgebra hereditária e $T=T_{1} \oplus T_{2} \oplus T_{3}$ um A-módulo inclinante parcial básico tal que $T_{1}, T_{2}$ e $T_{3}$ são A-módulos indecomponiveis. Se $\left(T_{1}, T_{2}\right)$ é uma sequência especial de $T_{1} \oplus T_{2}$ associada ao sistema estratificante $\left(X_{1}, X_{2}\right)$, onde $X_{2} ¥ T_{2}$, então $\left(T_{1}, T_{2}, T_{3}\right)$ é uma sequência especial de $T$ se, e somente se, existem sequências exatas da forma

$$
\begin{gathered}
0 \longrightarrow K_{1} \longrightarrow T_{3} \longrightarrow T_{1}^{n_{1}} \longrightarrow 0 \\
0 \longrightarrow K_{2} \longrightarrow K_{1} \longrightarrow X_{2}^{n_{2}} \longrightarrow 0
\end{gathered}
$$

onde $n_{1}=\operatorname{dim}_{K} \operatorname{Hom}_{A}\left(T_{3}, T_{1}\right)$ e $n_{2}=\operatorname{dim}_{K} \operatorname{Hom}_{A}\left(T_{3}, T_{2}\right)$. Neste caso $T$ está associado ao sistema estratificante $\left(X_{1}, X_{2}, K_{2}\right)$. 


\section{Referências Bibliográficas}

[1] I. Ágoston, D. Happel, E. Lukás and L. Unger. Finitistic dimension of standardly stratified algebras. Comm. Algebra 28 (2000), 144-160. 31

[2] I. Assem, D. Simson and A. Skowronski. Elements of Representation Theory of Associative Algebras. 1: Techniques of Representation Theory. Series: London Mathematical Society Student Texts 65. Cambridge University Press, 2006. 1, 2, 4, 7, 9, 13, 14, 15, 17, 18, 31, 32, $33,34,37,39,55,56,63,69,75,76$

[3] M. Auslander, I. Rieten and S. Smalø. Representation Theory of Artin Algebras. Cambridge Studies in Avanced Mathemathics 36. Cambridge Academic University Press, 1995.

[4] A. I. Bondal and M. M. Kapranov, Representable functors, Serre functors and reconstructions, Izv. Akad. Nauk SSSR Ser. Mat. 53 (1989), no. 6, 1183-1205, 1337; Translation in Math. USSR-Izv. 35 (1990), no.3, 519-541. ix, 32

[5] P. Cadavid. Álgebras estandarmente estratificadas e álgebras quase-hereditárias. Dissertação de mestrado. IME-USP. 2007. 27

[6] W. Crawley-Boevey. Exceptional sequences of representations of quivers. Proceedings of ICRA VI, Carleton-Ottawa Math. LNS Vol. 14 (1992), 117-124. ix, x, 23, 32, 36

[7] K. Erdman and C. Saenz, On standardly stratified algebras. Comm. in algebra Vol. 31, No. 7, 3446-3446 (2003). ix, 23, 24, 25

[8] Yu. A. Drozd and V. V. Kirichenko. Finite dimensional algebras. Apendix on quasihereditary algebras by V. Dlab. Springer-Verlang, Berlin, 1994. 29, 31

[9] A. L. Gorodentsev and A. N. Rudakov, Exceptional vector bundles on projective spaces, Duke. math. J. 54 (1987), 115-130. ix, 31

[10] E. Marcos, O. Mendoza and C. Sáenz, Stratifying systems via relative projetive modules. Comm. Algebra 33 (2005), no.5, 1559- 1573. ix, 23

[11] E. Marcos, O. Mendoza and C. Sáenz, Stratifying systems via relative simple modules. Comm. Algebra 280 (2004), no. 2, 472-487. ix, 23, 24, 25, 26, 34, 35 
[12] E. Marcos, O. Mendoza and C. Sáenz, Applications of stratifying systems to the finitistic dimension. J. Pure App. Algebra 205 (2006), no. 2, 393-411. ix

[13] E. Marcos, O. Mendoza, C. Sáenz and R. Zuazua, Quadratic forms associated to stratifying systems. J. of Algebra 302 (2006), no. 2, 750-770. ix

[14] C. M. Ringel. The braid group action on the set of exceptional sequences of a hereditary artin algebra. Contemp. Math. 1994, 171, 339-352. Abelian group theory and related topics, Proc. Oberwolfach Conf. 1993. ix, 32

[15] C. M. Ringel. The category of good modules over a quasi-hereditary algebras has almost split sequences. Proceedings of the Sixth International Conference on Representations of Algebras (Ottawa, ON, 1992), 17 pp., Carleton-Ottawa Math. Lecture Note Ser., 14, Carleton Univ., Ottawa, ON, 1992. 25, 27, 85

[16] A. N. Rudakov, Execptional collections, mutations and helices, Helicesand vector bundles, 1-6, London Mat. Soc. Lecture Note Ser., 148, Cambridge Univ. Pres, Cambridge, 1990. ix, 32

[17] D. Simson and A. Skowronski. Elements of Representation Theory of Associative Algebras. Vol 2: Tubes and Conceladed Algebras of Euclidean type. Series: London Mathematical Society Student Texts 71. Cambridge University Press, 2007. 1, 12, 18, 21, 44, 47, 48, 49, 50

[18] D. Simson and A. Skowronski. Elements of Representation Theory of Associative Algebras. Vol 3: Representation-Infinite Tilted Algebras. Series: London Mathematical Society Student Texts 72. Cambridge University Press, 2007. 37, 44, 45

[19] A. Schonfield, Semi invariants of quivers, J. London Math. Soc. 43 (1991), 385-395.

[20] U. Seidel, Exceptional sequences for quivers of Dynkin type, Com. in algebra 29 (3) (2001), 1373-1386. 32

[21] C. Xi. Standardly stratified algebras and cellular algebras. Math. Proc. Cambridge Philos. Soc. 133 (2002), no 1, 37-53. 27, 29 


\section{Índice Remissivo}

classe de torção, 39

classe livre de torção, 39

fórmulas de Auslander , 10

par de torçao, 39

apresentação, 3

Auslander-Reiten

carcás de, 8

funtor de translação de, 10

sequência de, 7

teorema de, 7

avaliação, 4

boca, 19

caminho(s), 2

categoria de, 19

álgebra de, 2

carcás, 2

acíclico, 2

com relações, 3

com translação, 18

conexo, 2

de Auslander-Reiten, 8

grafo subjacente do, 2

limitado, 3

localmente finito, 18

ordinário, 3

representação do, 4

Cartan

matriz de, 12

categoria

de caminhos, 19

injetivamente estável, 9

mesh, 20

projetivamente estável, 9

ciclo, 2

começo, 2 componente

auto hereditária, 20

estândar, 20

conjunto completo de idempotentes primitivos ortogonais, 1

Coxeter

matriz de, 17

transformação de, 17

diagramas

de Dynkin, 14

euclidianos, 15

canonicamente orientados, 16

Dynkin

diagramas de, 14

elemento(s)

mesh, 20

relativamente simples, 25

epimorfismo

que cinde, 7

euclidianos

diagramas, 15

filtração, 24

final, 2

flecha, 2

começo de uma, 2

final de uma, 2

funtor

de translação de Auslander-Reiten, 10

Gabriel

teorema de, 3,15

grafo subjacente, 2

Grothendieck

grupo de, 12, 26

grupo

de Grothendieck, 12, 26 
hereditária

álgebra, 13, 14

homomorfismo

irredutível, 7

ideal admissível, 3

idempotentes primitivos ortogonais conjunto completo de, 1

irredutível

homomorfismo, 7

momomorfismo, 7

Kronecker

álgebra de, 5

matriz

de Cartan, 12

de Coxeter, 17

mesh

categoria, 20

elemento, 20

monomorfismo

irredutível, 7

espaço de, 8

que cinde, 7

módulo

estândar, 28

excepcional, 32

inclinante, 33

generalizado, 29

parcial, 33

pré-injetivo, 14

pós-projetivo, 14

regular, 14

objeto

relativamente injetivo, 26

relativamente projetivo, 26

simples, 21

predecessor imediato, 18

relativamente simples

elementos, 25

sistema estratificante via módulos, 25

relação, 3

representação

do carcás, 4

retração, 7 sequência

de Auslander-Reiten, 7

excepcional, 32

quase-cindida, 7

seção, 7

sistema estratificante, 24

completo, 34

estândar, 25

via módulos relativamente injetivos, 24

via módulos relativamente simples, 24,25

sucessor imediato, 18

teorema

de Auslander-Reiten, 7

de Gabriel, 3, 15

tijolo, 21

transformação

de Coxeter, 17

traço, 27

tubo

auto hereditário, 20

estável, 19

hereditário, 20

homogêneo, 19

vetor

dimensão, 11

vértice, 2

álgebra

basica, 1

conexa, 1

de caminhos, 2

de Kronecker, 5

estandarmente estratificada, 28

hereditária, 13, 14

indecomponivel, 1

quase-hereditária, 31 